\title{
Invading Escherichia coli Genetics with a Xenobiotic Nucleic Acid Carrying an Acyclic Phosphonate Backbone (ZNA)
}

Min Luo, ${ }^{\S}$ Elisabetta Groaz, ${ }^{\S}$ Mathy Froeyen, ${ }^{\S}$ Valérie Pezo, ${ }^{\ddagger}$ Faten Jaziri, ${ }^{\ddagger}$, , Piotr Leonczak,${ }^{\S}$ Guy Schepers,${ }^{\S}$ Jef Rozenski, ${ }^{\S}$ Philippe Marlière,${ }^{\ddagger}$ and Piet Herdewijn ${ }^{\S, *}$

$\S$ KU Leuven, Rega Institute for Medical Research, Medicinal Chemistry, Herestraat 49 - box1041, 3000 Leuven, Belgium

*énomique Métabolique, Genoscope, Institut François Jacob, CEA, CNRS, Univ Evry, Université Paris-Saclay, 2 Rue Gaston Crémieux 91057 Evry, France

Table of Contents

1. NMR Spectra of Compounds 


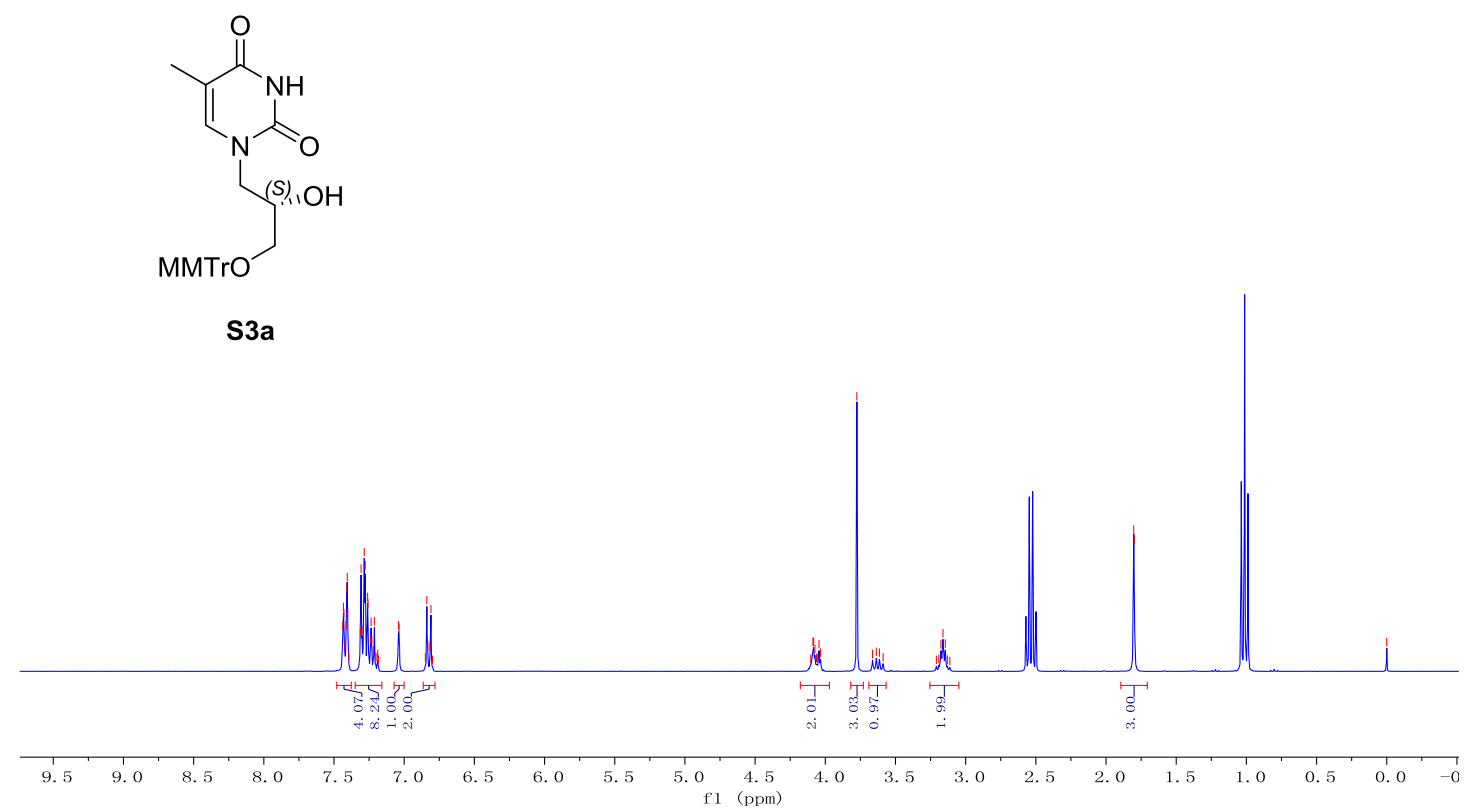

Solvent $\mathrm{CDCl} 3$

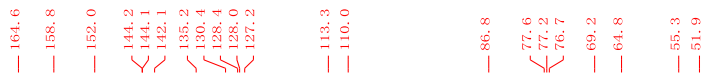

Nucleus 13C

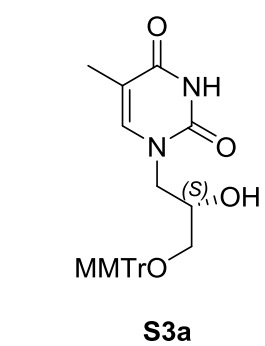

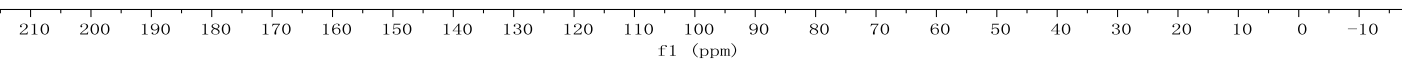




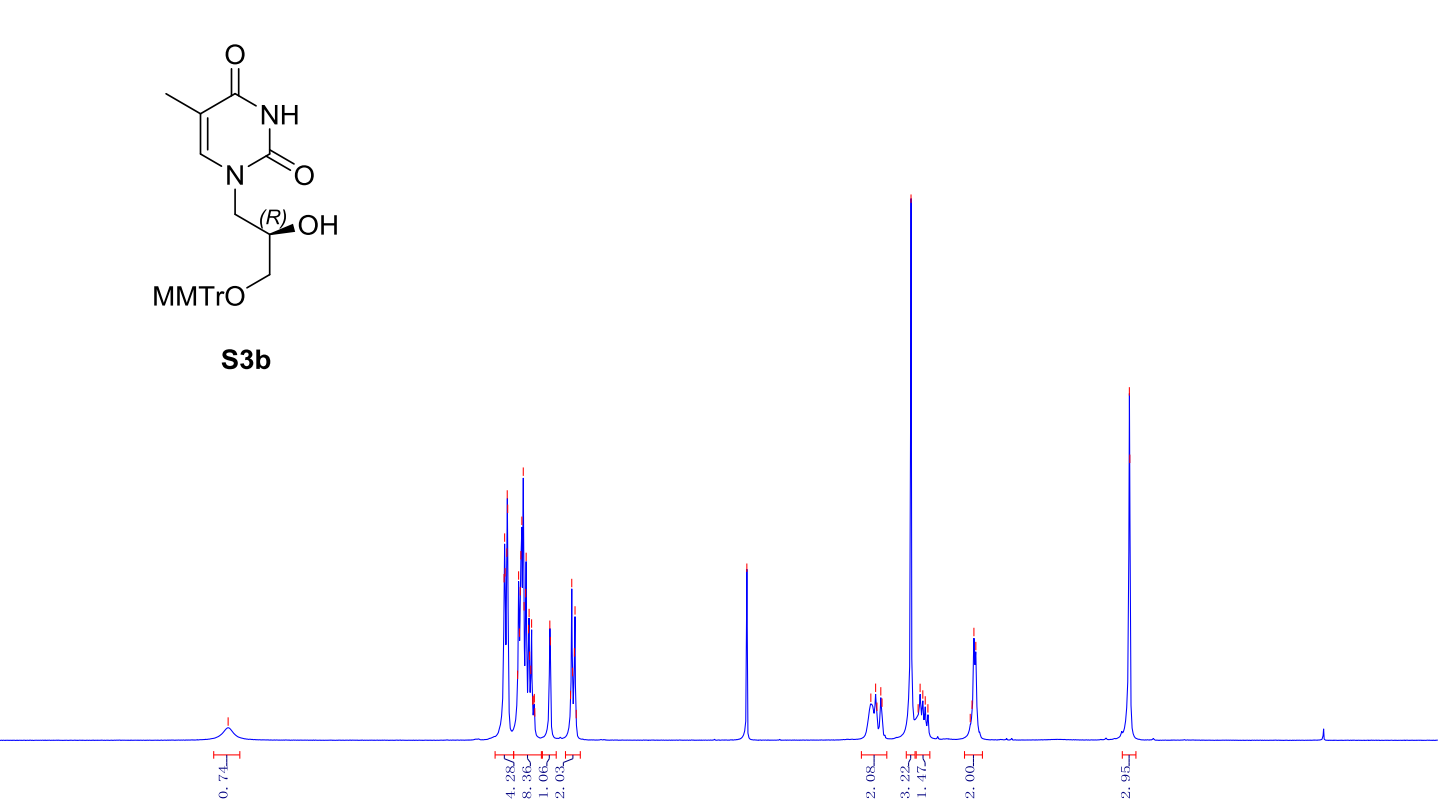

$\begin{array}{lllllllllllllllllllllllllllllllll}2.0 & 11.5 & 11.0 & 10.5 & 10.0 & 9.5 & 9.0 & 8.5 & 8.0 & 7.5 & 7.0 & 6.5 & 6.0 & 5.5 & 5.0 & 4.5 & 4.0 & 3.5 & 3.0 & 2.5 & 2.0 & 1.5 & 1.0 & 0.5 & 0.0 & -0.5 & -1\end{array}$

Solvent $\mathrm{CDCl} 3$

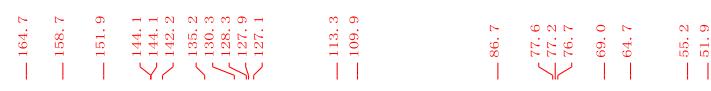

Nucleus $13 \mathrm{C}$

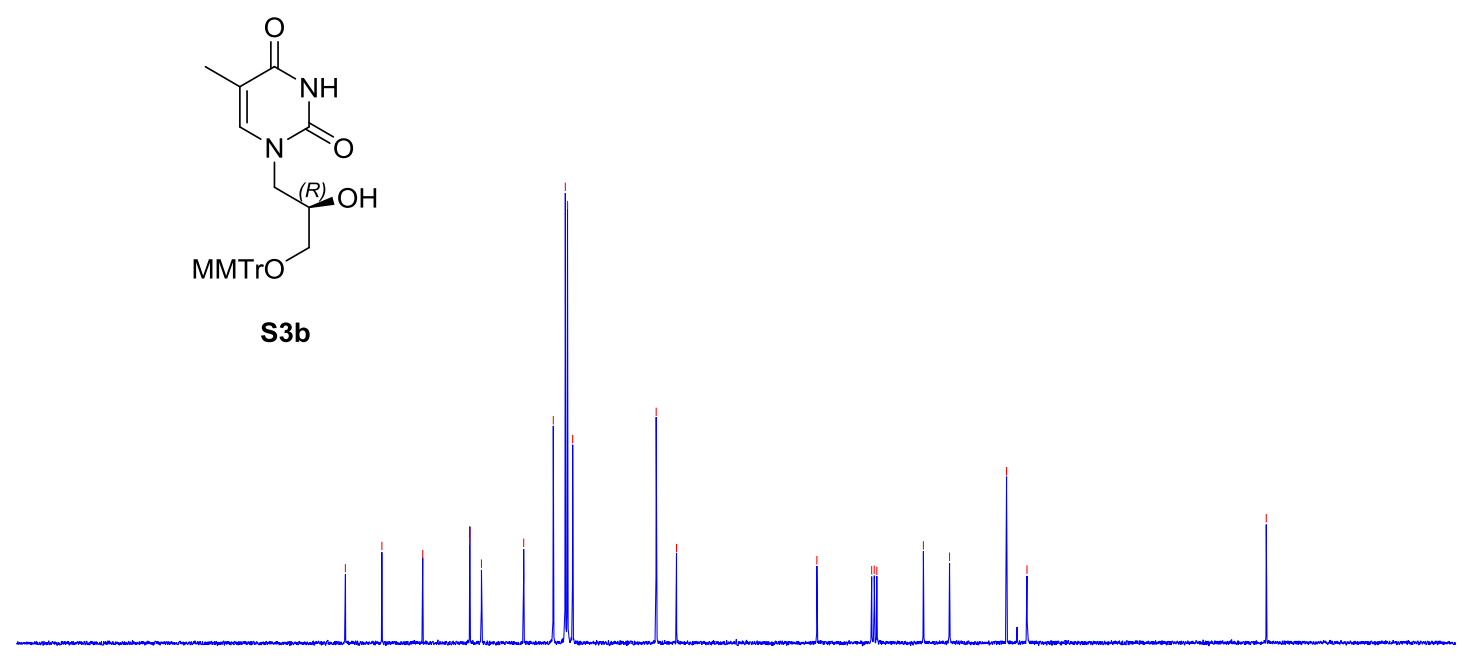

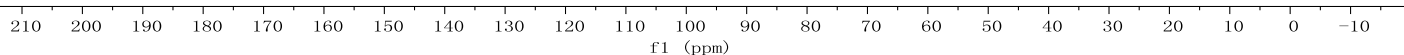




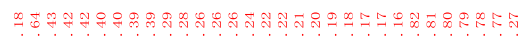
Solvent CDCl3

Nucleus $1 \mathrm{H}$<smiles>COC[C@H](O)Cn1cnc2c(N)ncnc21</smiles>

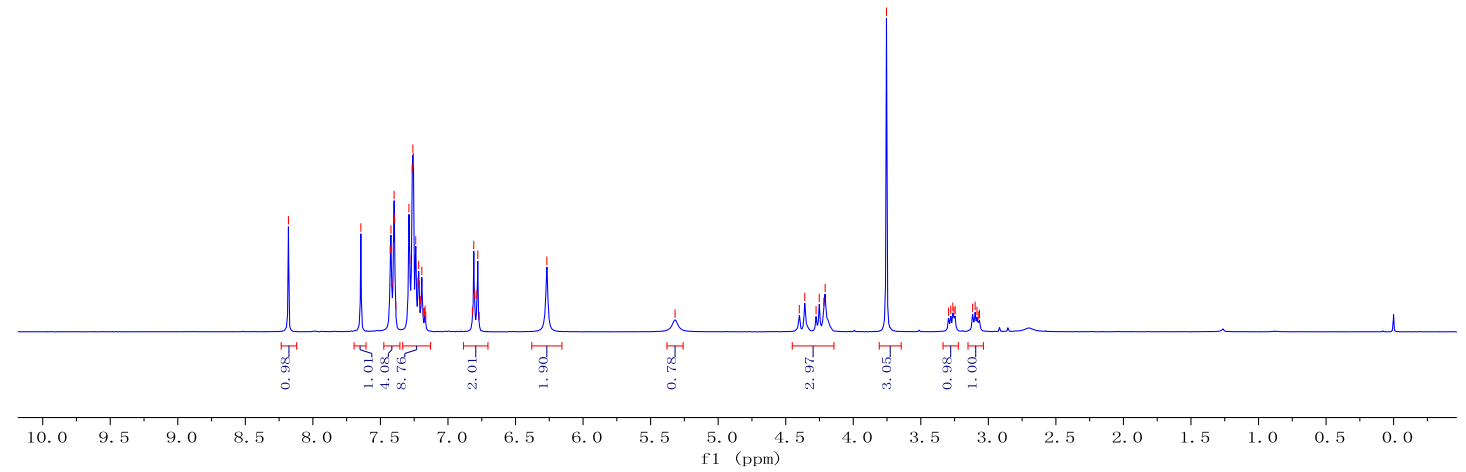

Solvent $\mathrm{CDCl} 3$

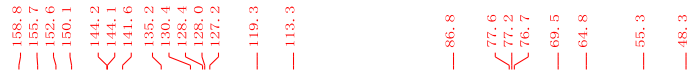

Nucleus 13C

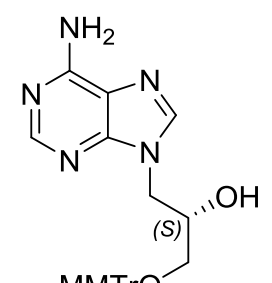

S4a

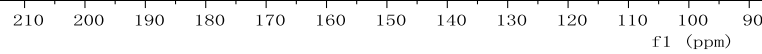




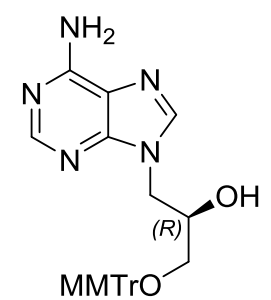

S4b

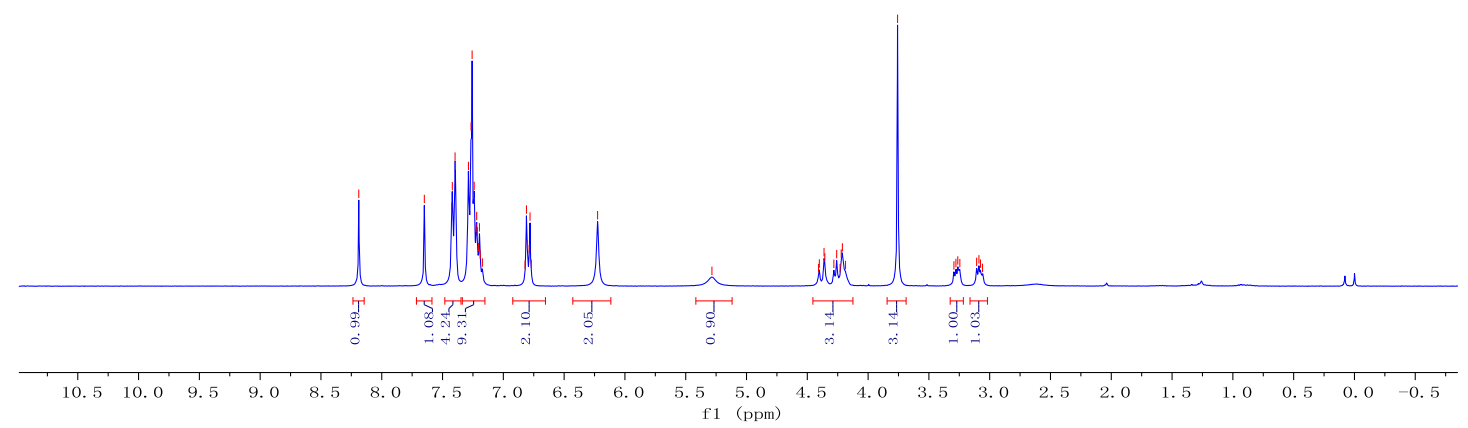

Solvent $\mathrm{CDCl} 3$

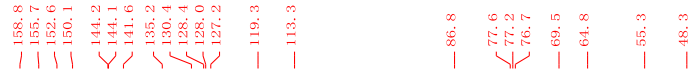

Nucleus 13C

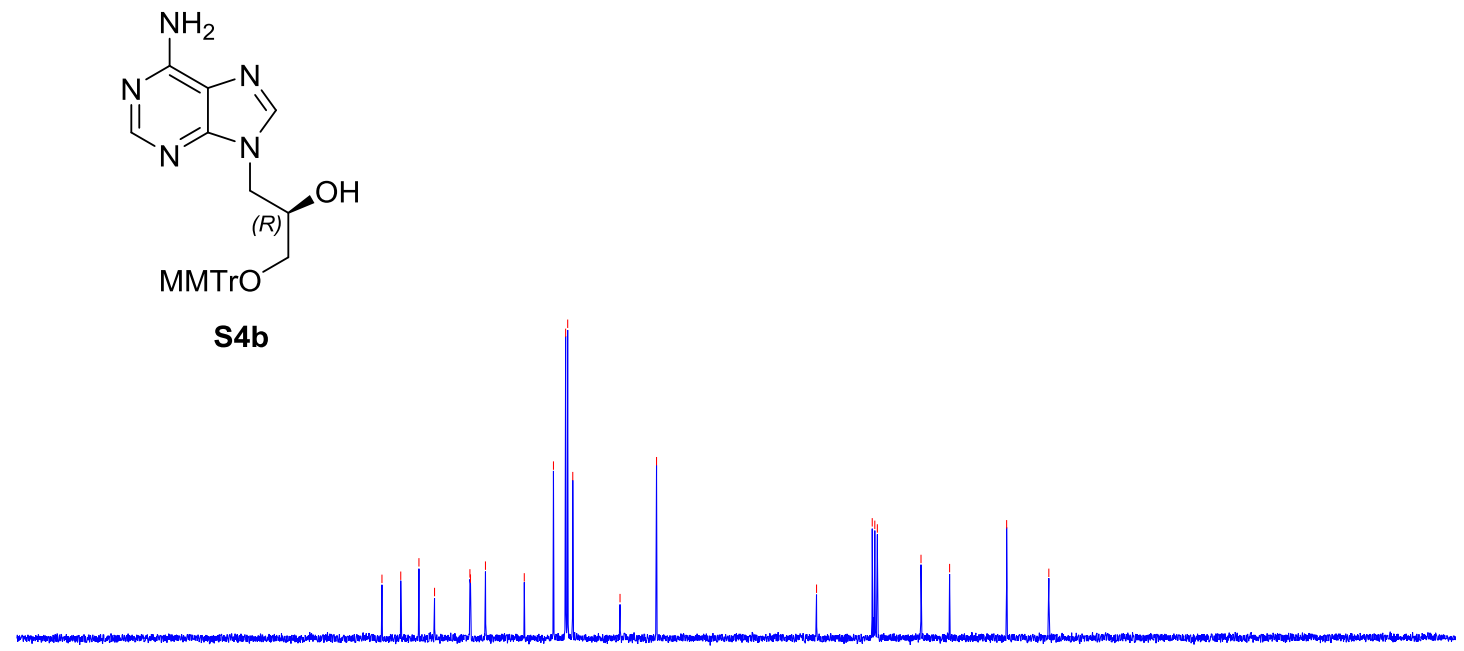

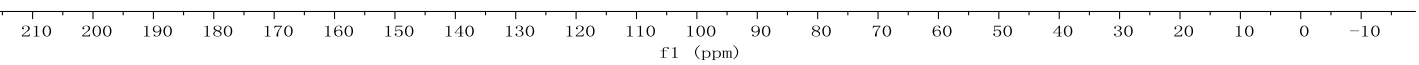




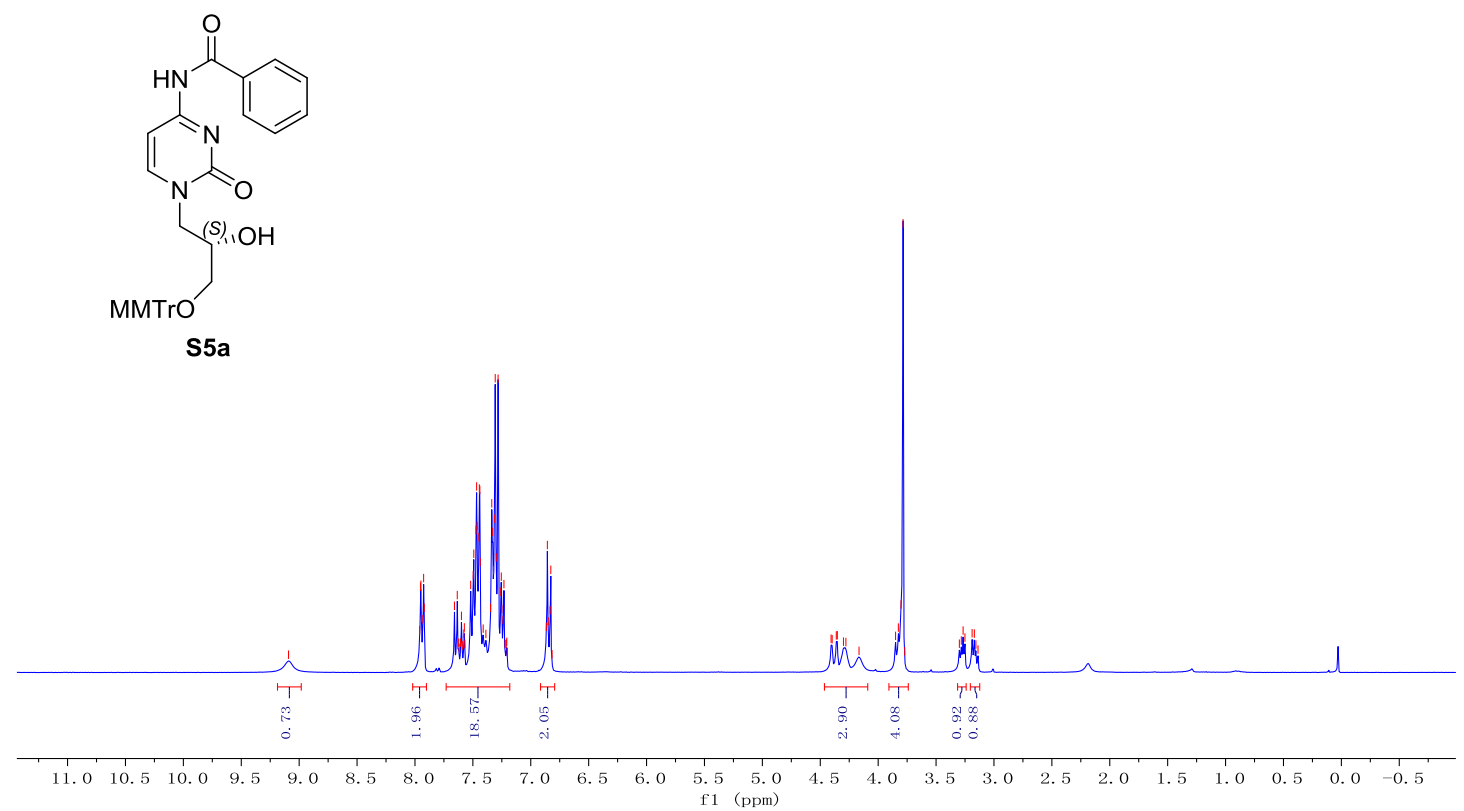

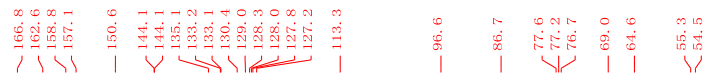

Solvent $\mathrm{CDCl} 3$

Nucleus 13C

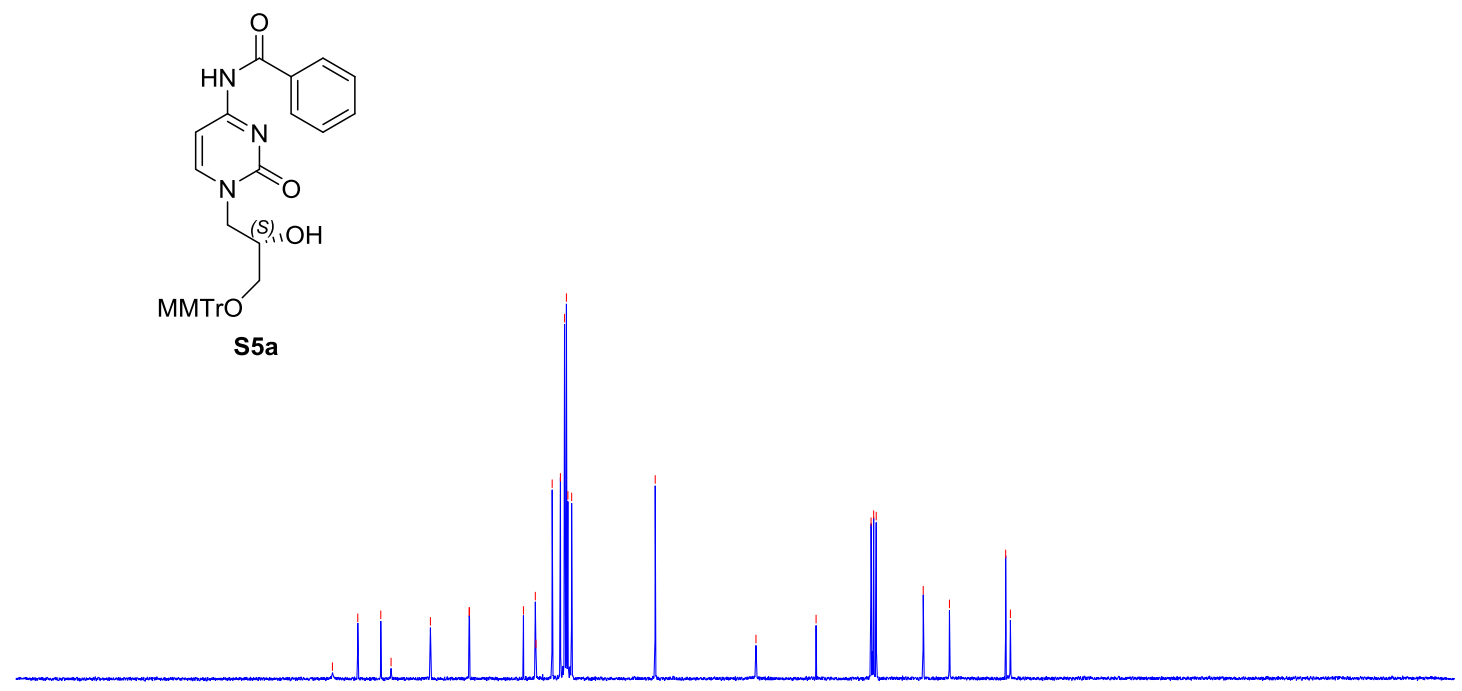

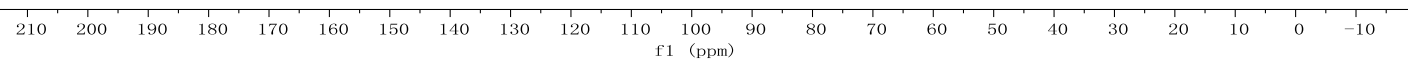


<smiles>COCC(O)Cn1ccnc2c(=O)[nH]c(=O)c-2c1</smiles>

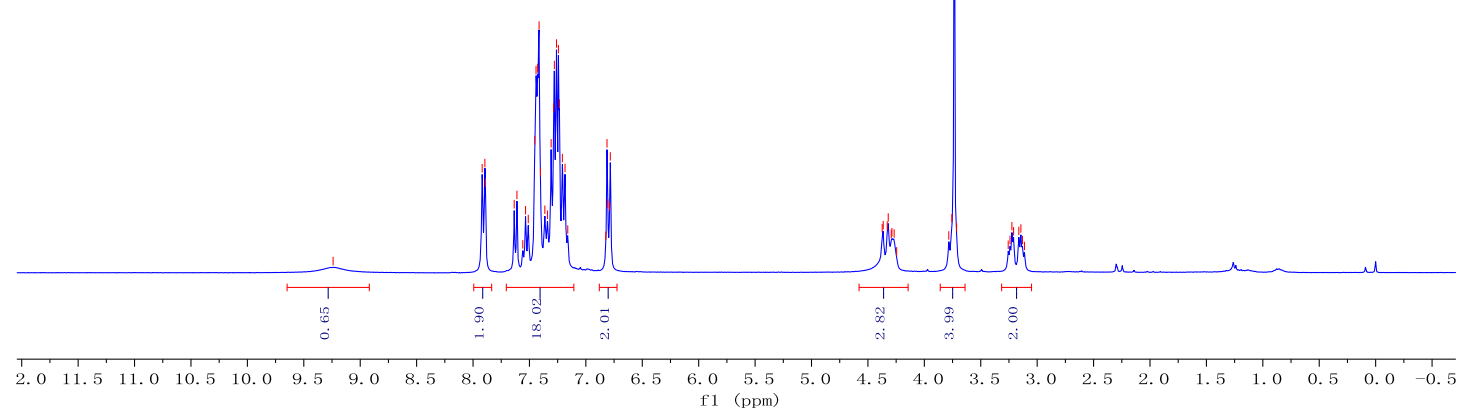

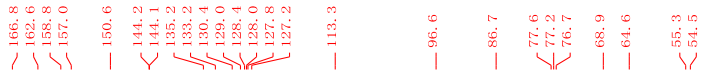

Solvent $\mathrm{CDCl} 3$

Nucleus 13C

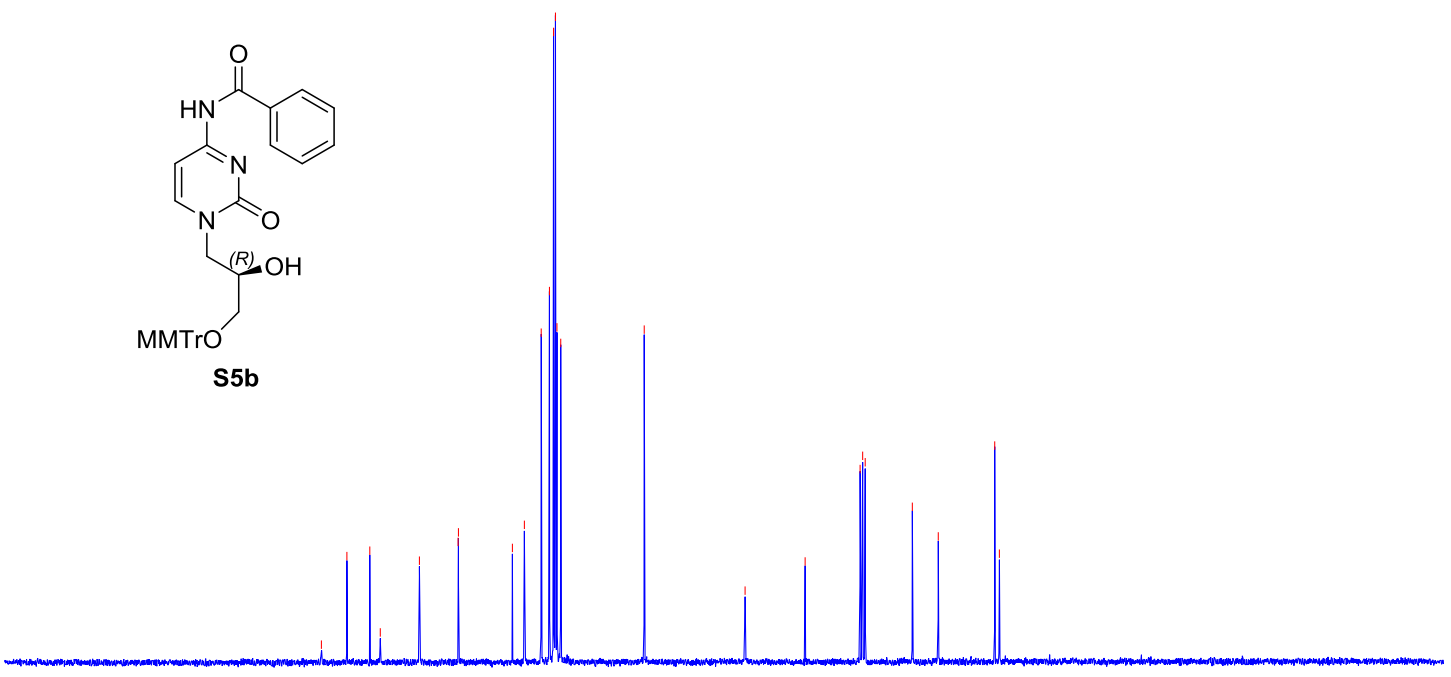

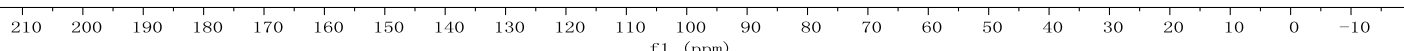



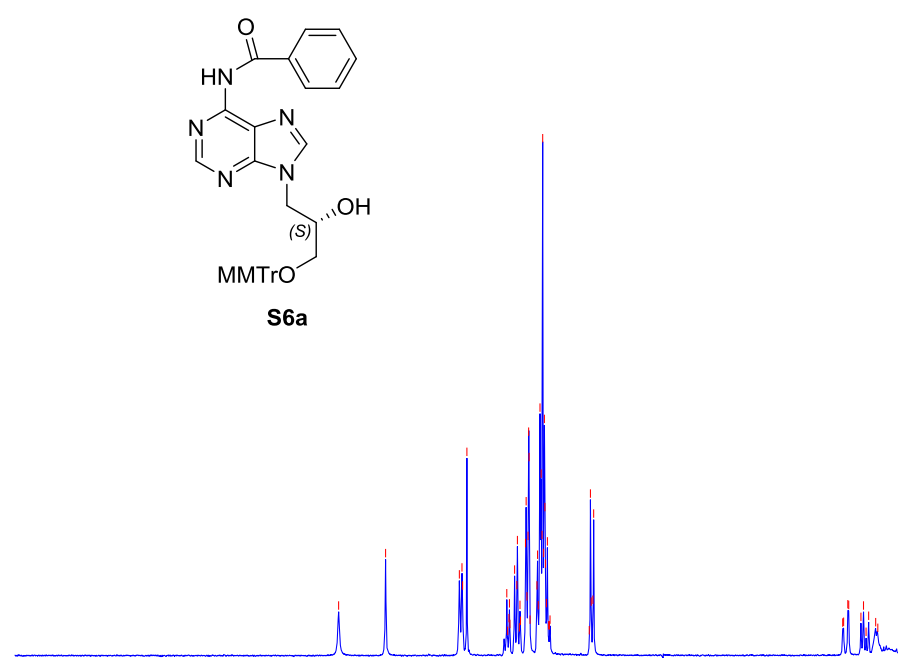

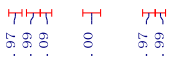

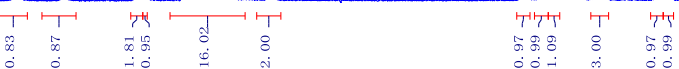
$\begin{array}{llllllllllllllllllllllllllllllllllllllllll}12.0 & 11.5 & 11.0 & 10.5 & 10.0 & 9.5 & 9.0 & 8.5 & 8.0 & 7.5 & 7.0 & 6.5 & 6.0 & 5.5 & 5.0 & 4.5 & 4.0 & 3.5 & 3.0 & 2.5 & 2.0 & 1.5 & 1.0 & 0.5 & 0.0 & -0.5 & -1 .\end{array}$

Solvent $\mathrm{CDCl} 3$

|

Nucleus 13C

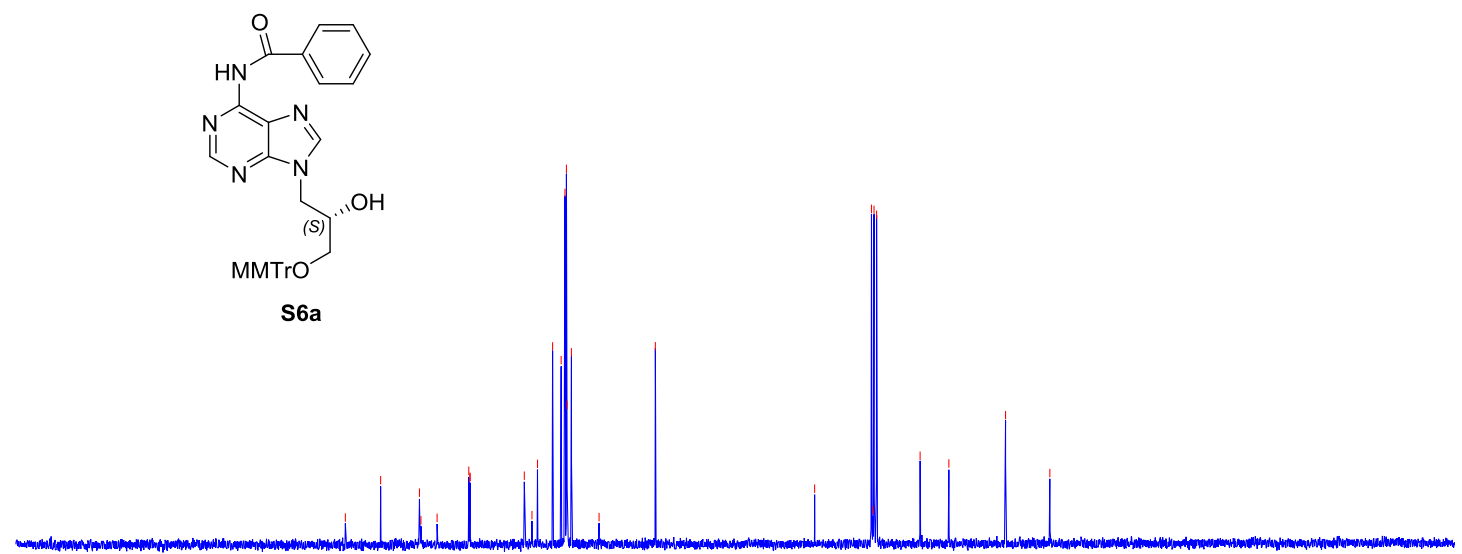

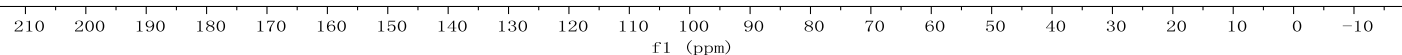




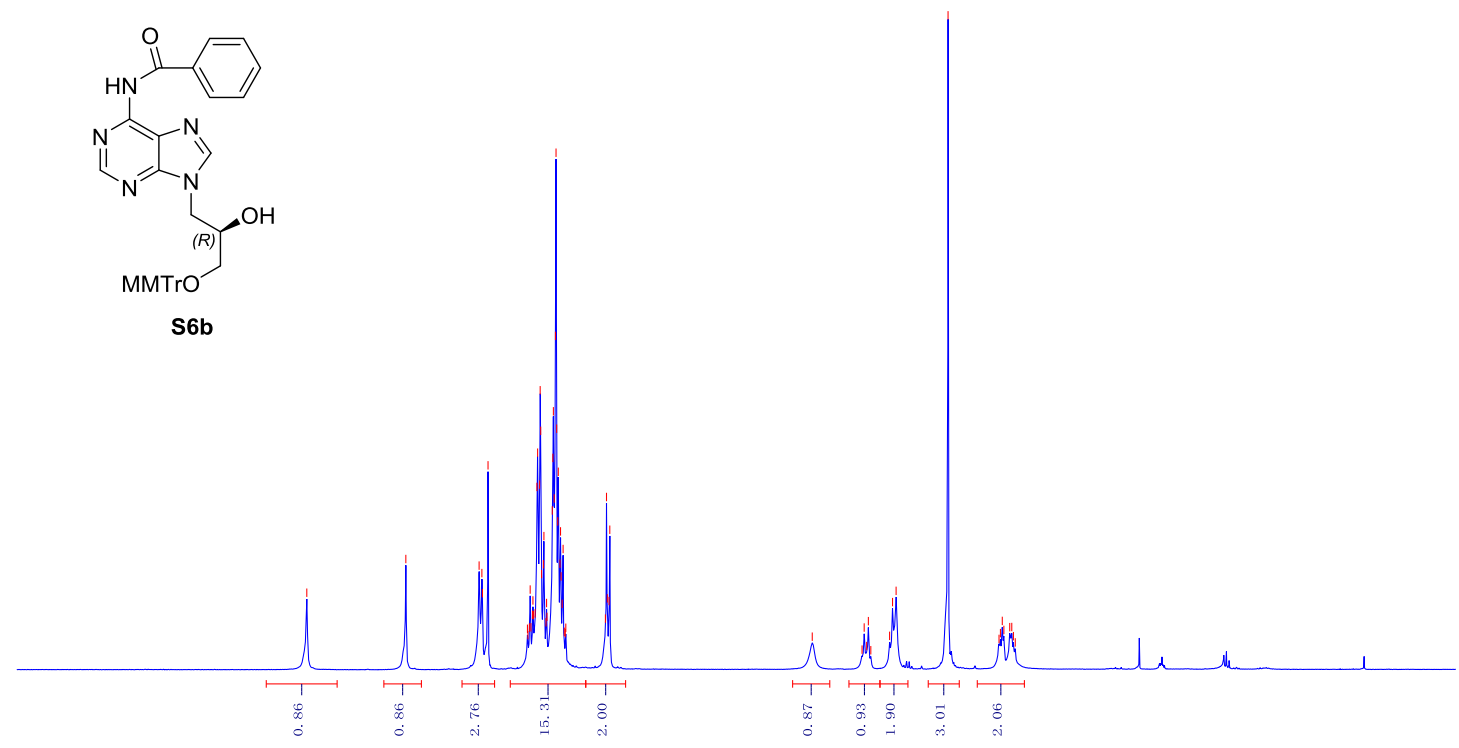

$\begin{array}{llllllllllllllllllllllllllll}2.0 & 11.5 & 11.0 & 10.5 & 10.0 & 9.5 & 9.0 & 8.5 & 8.0 & 7.5 & 7.0 & 6.5 & 6.0 & 5.5 & 5.0 & 4.5 & 4.0 & 3.5 & 3.0 & 2.5 & 2.0 & 1.5 & 1.0 & 0.5 & 0.0 & -0.5\end{array}$

Nucleus 13C

|

Solvent $\mathrm{CDCl} 3$

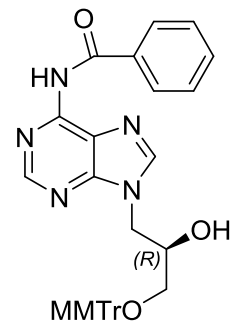

s6b

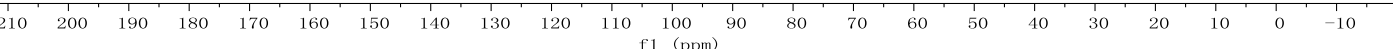




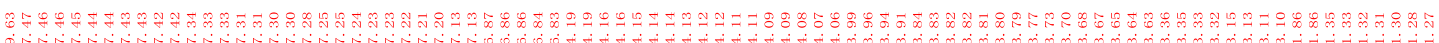
Solvent CDCl3

Nucleus $1 \mathrm{H}$

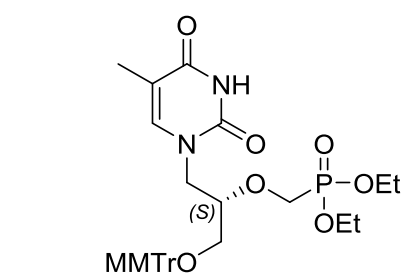

S7a

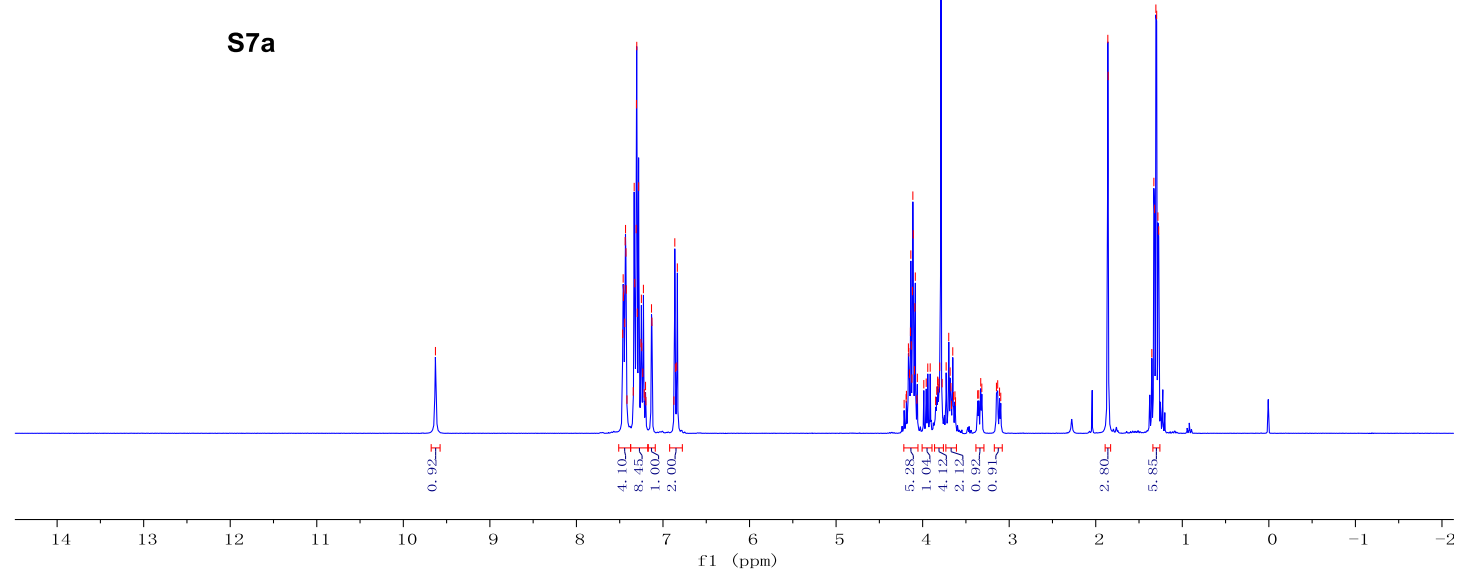

Solvent CDCl
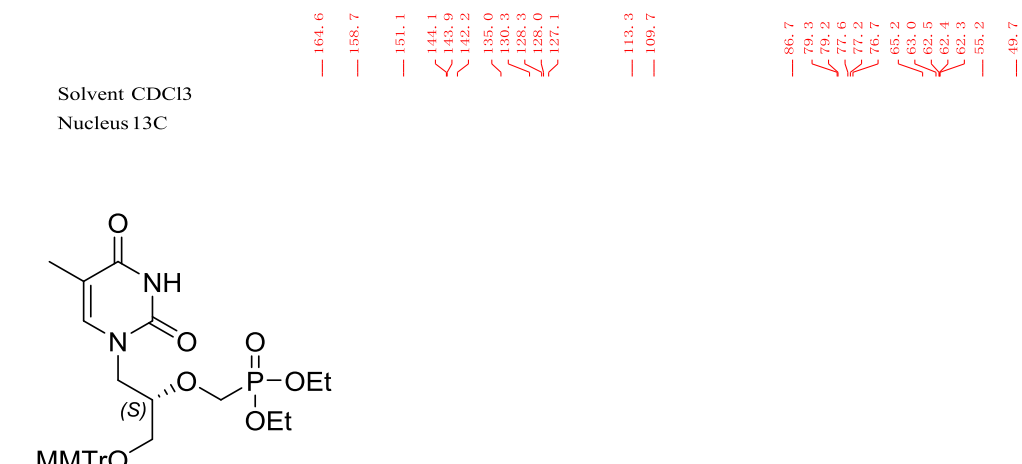

S7a

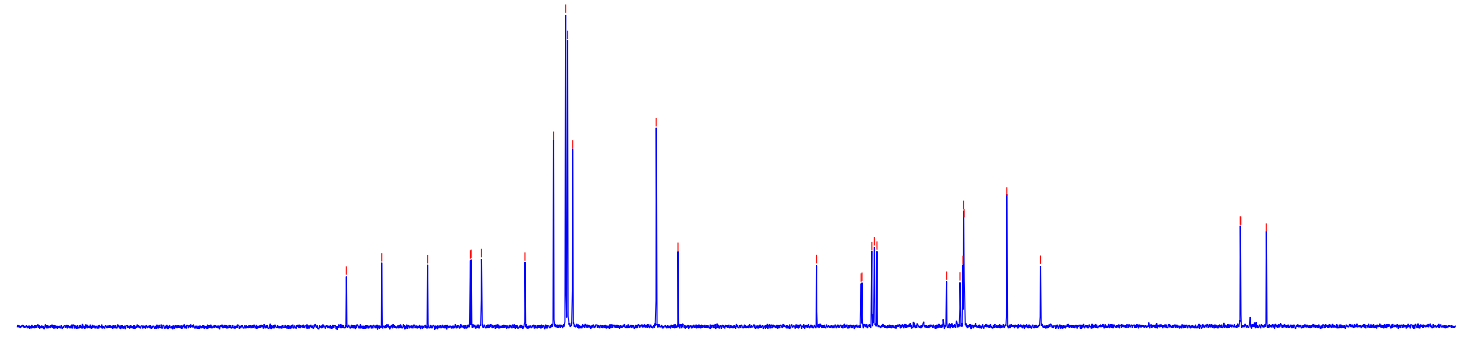

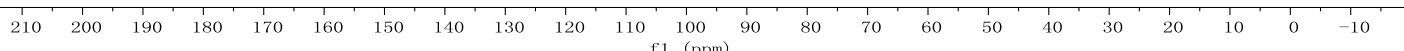




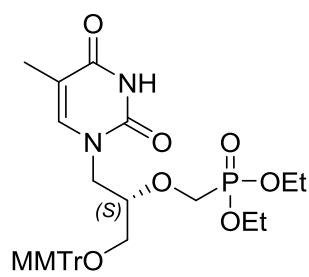

S7a

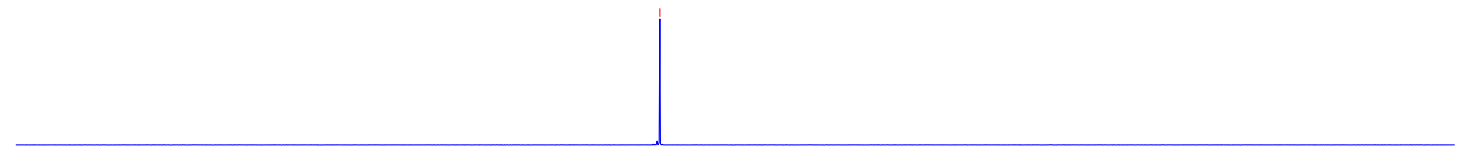

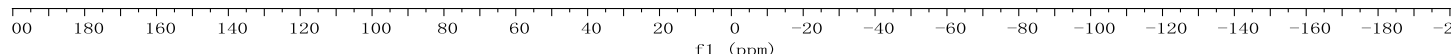

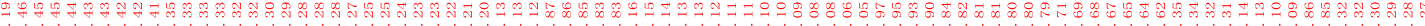
Solvent $\mathrm{CDCl} 3$

Nucleus $1 \mathrm{H}$
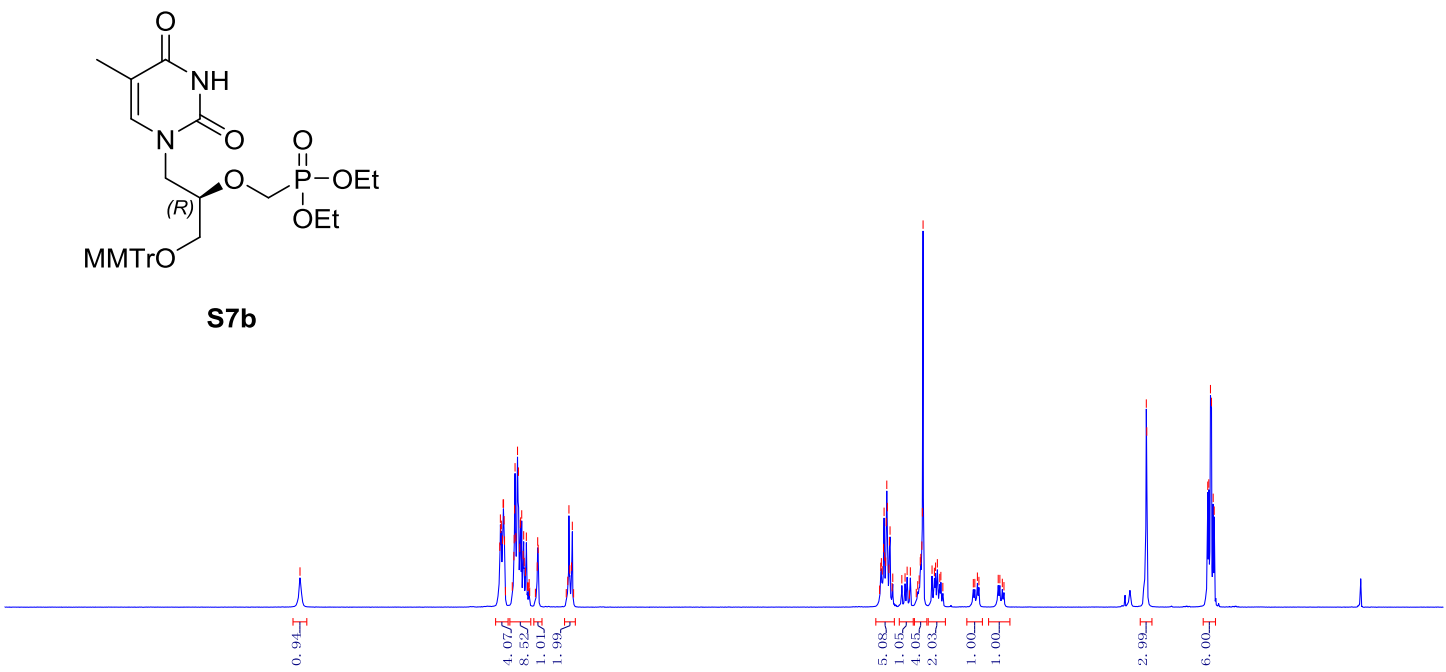

$\begin{array}{llllllllllllllllllllllllllllllllllllllll}11.5 & 11.0 & 10.5 & 10.0 & 9.5 & 9.0 & 8.5 & 8.0 & 7.5 & 7.0 & 6.5 & 6.0 & 5.5 & 5.0 & 4.5 & 4.0 & 3.5 & 3.0 & 2.5 & 2.0 & 1.5 & 1.0 & 0.5 & 0.0 & -0.5\end{array}$ 
Nucleus 13C

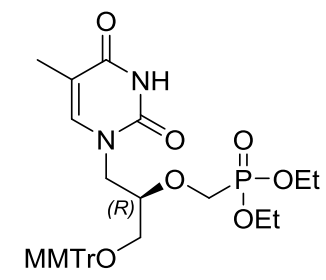

S7b

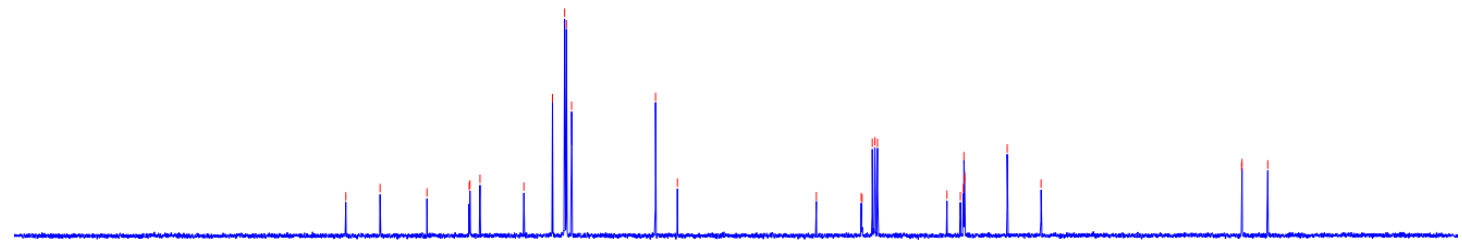

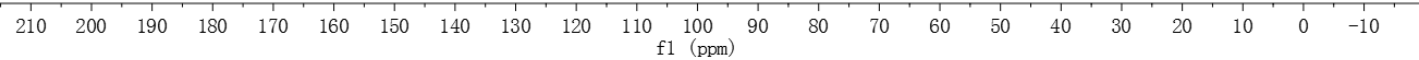

Solvent $\mathrm{CDCl} 3$

Nucleus 31P

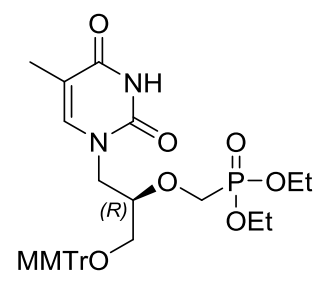

S7b

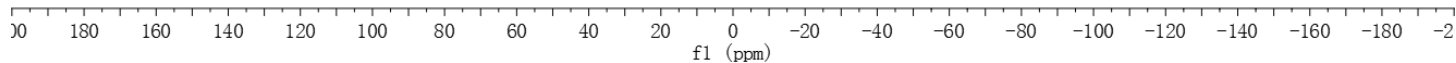



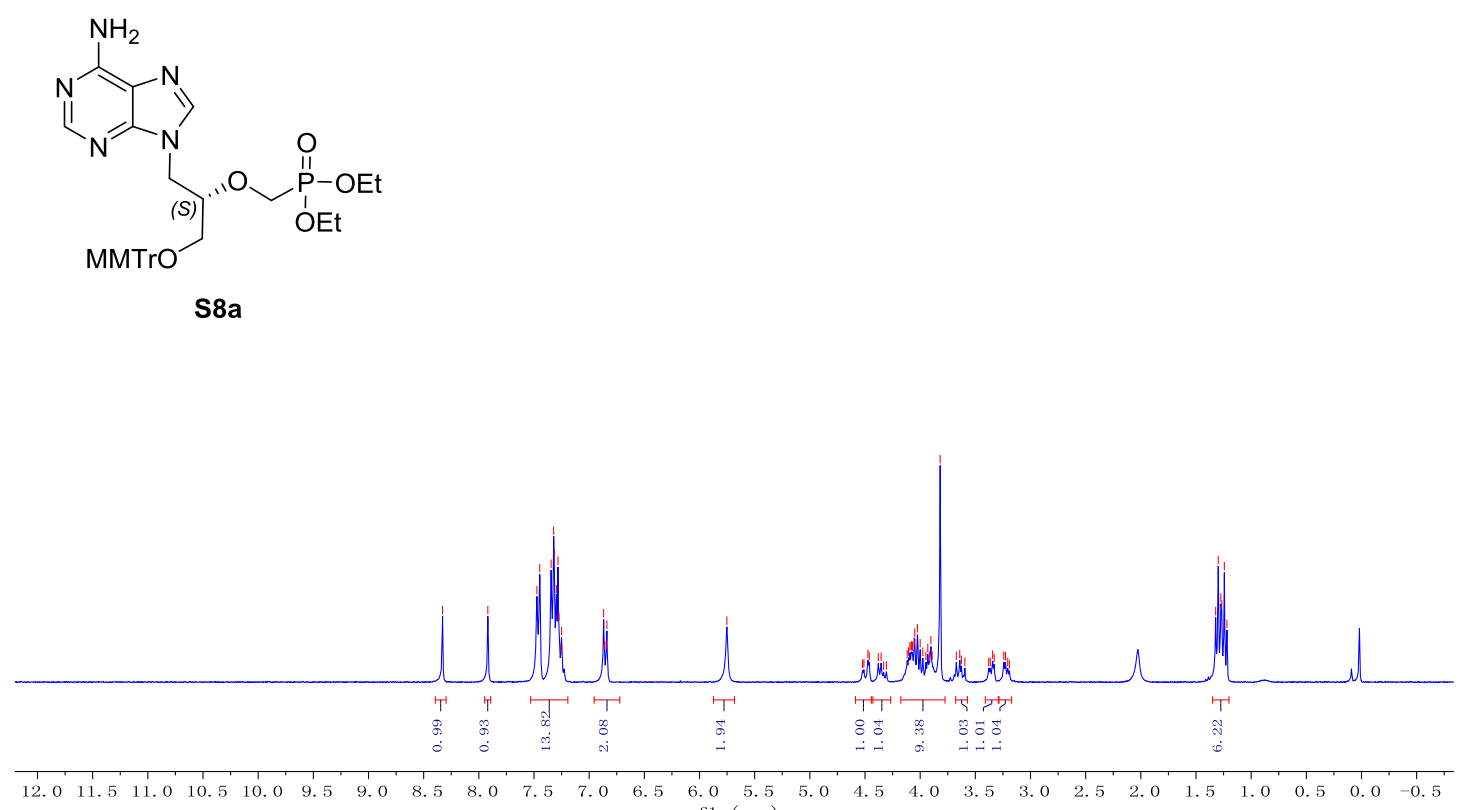

Solvent $\mathrm{CDCl} 3$

Nucleus 13C

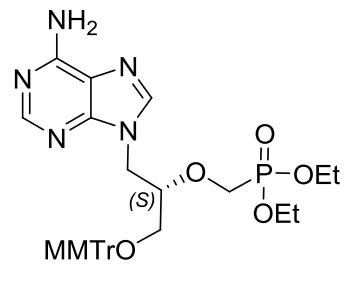

S8a

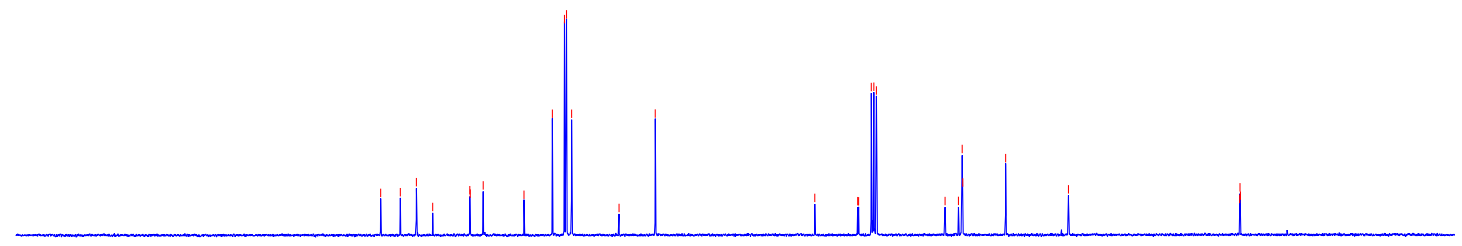

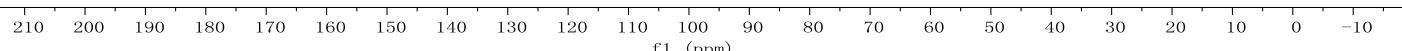


Solvent CDC13

Nucleus 31P
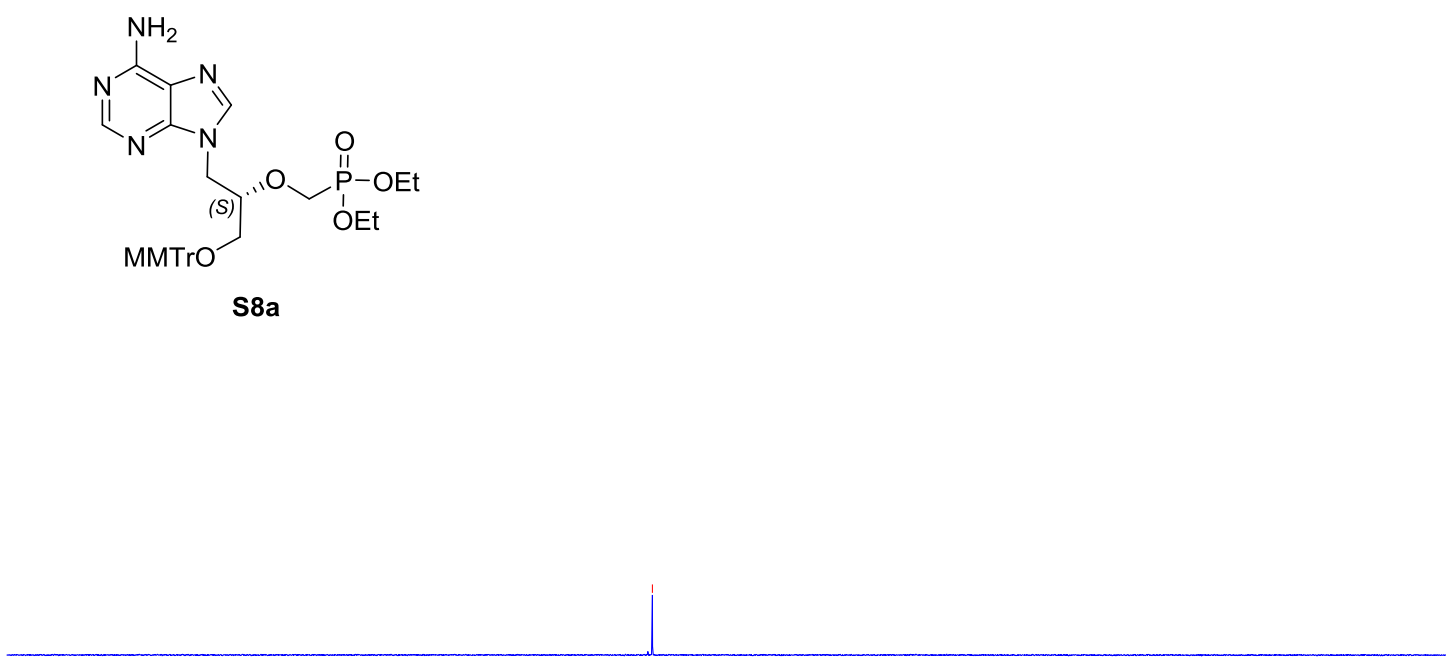

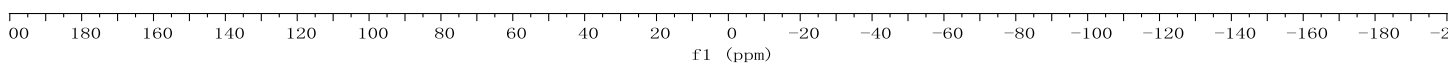

Solvent CDC13

Nucleus $1 \mathrm{H}$

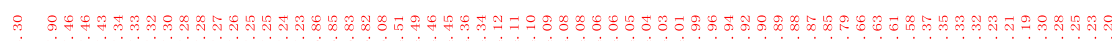<smiles>CCOC[C@H](Cn1cnc2c(N)ncnc21)OCP(=O)(OCC)OCC</smiles>

S8b

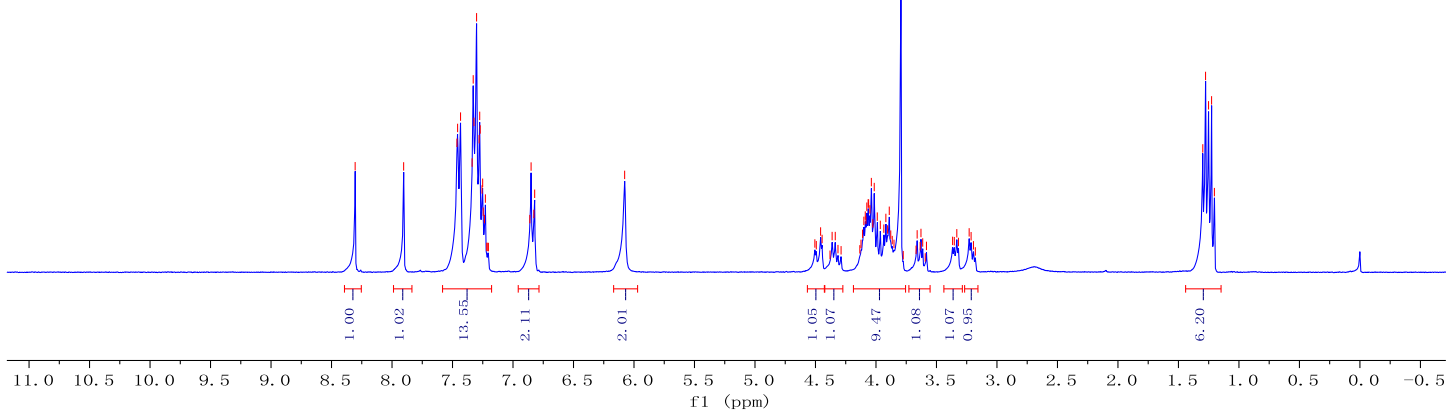

S14 

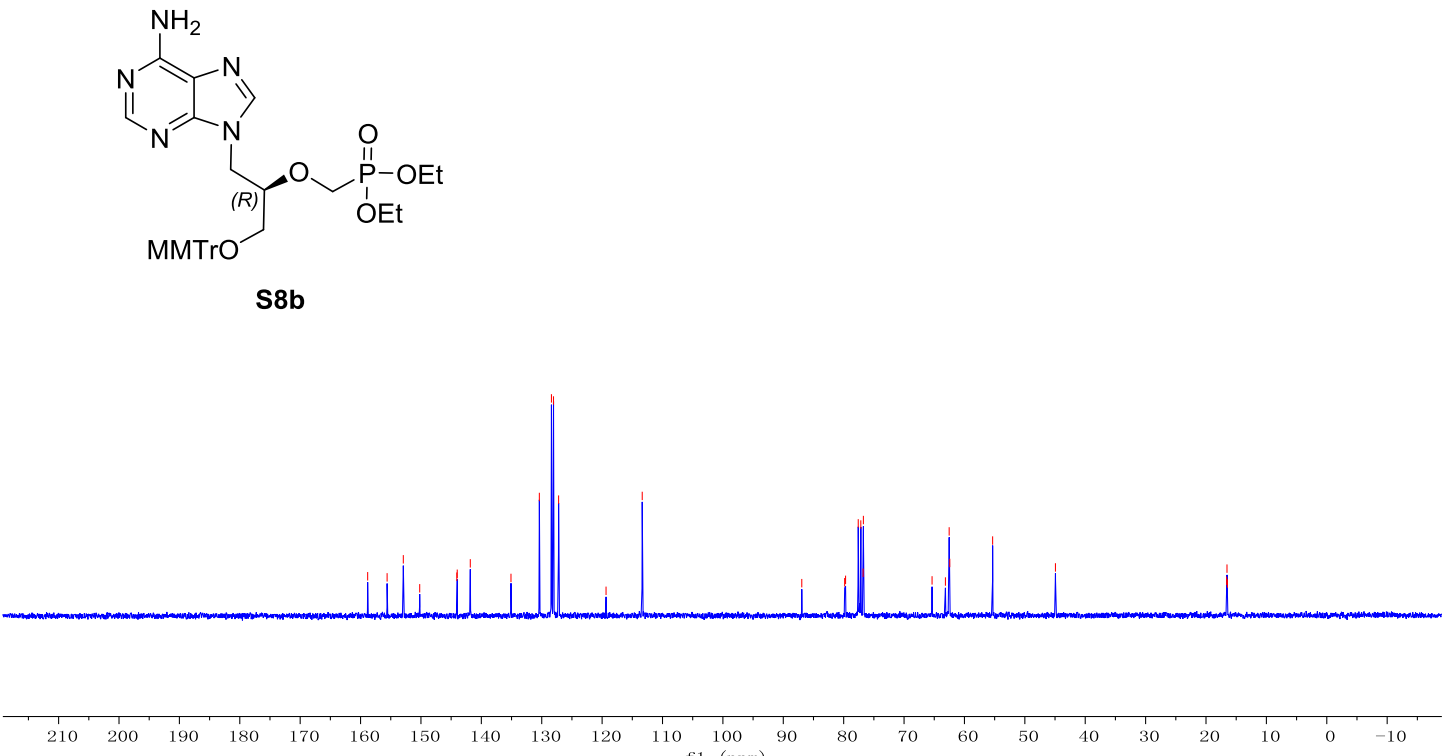

Solvent $\mathrm{CDCl} 3$

Nucleus 31P

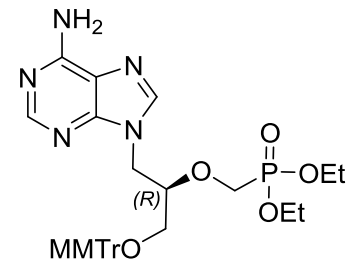

S8b

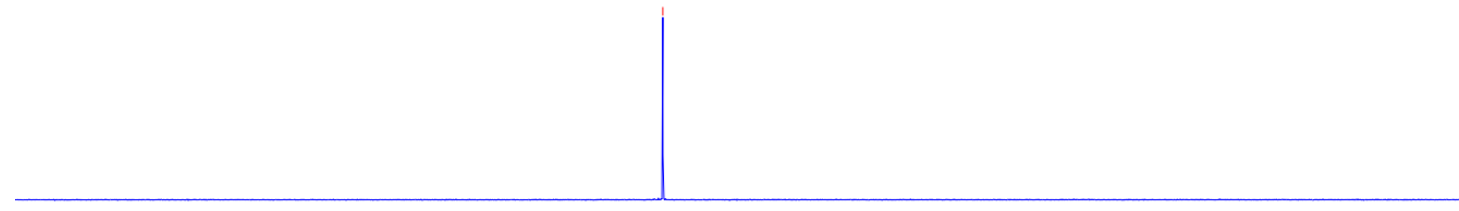

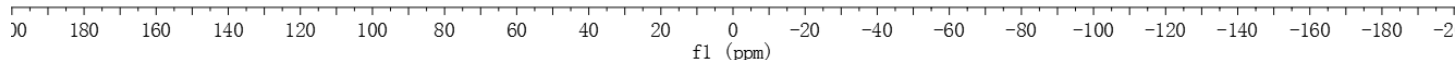



Solvent $\mathrm{CDCl} 3 \mathrm{C}$

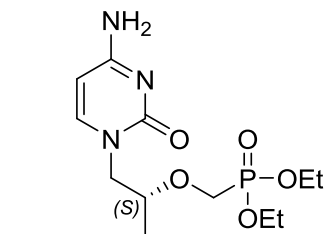

MMTrO

S9a

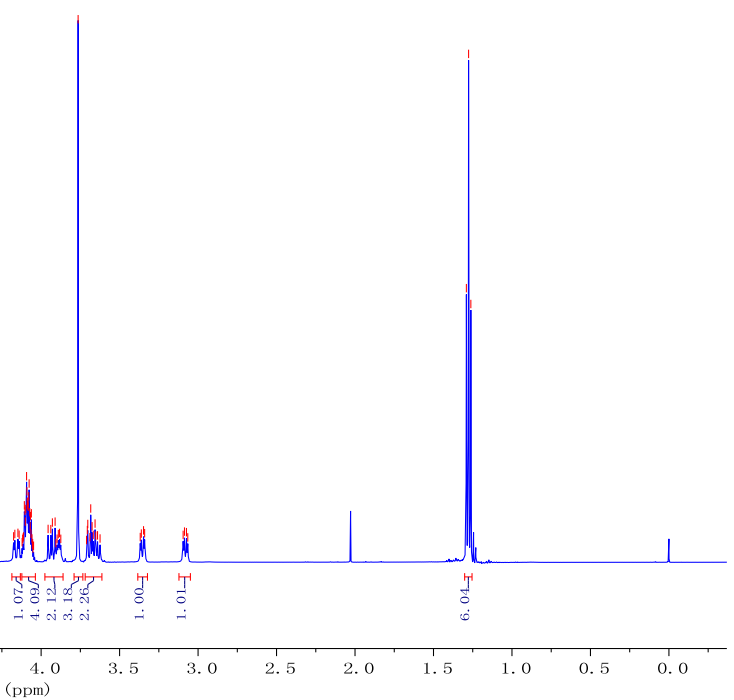

Nucleus 13C<smiles>CCOC[C@H](COC)OCP(=O)(OCC)OCC</smiles>

S9a

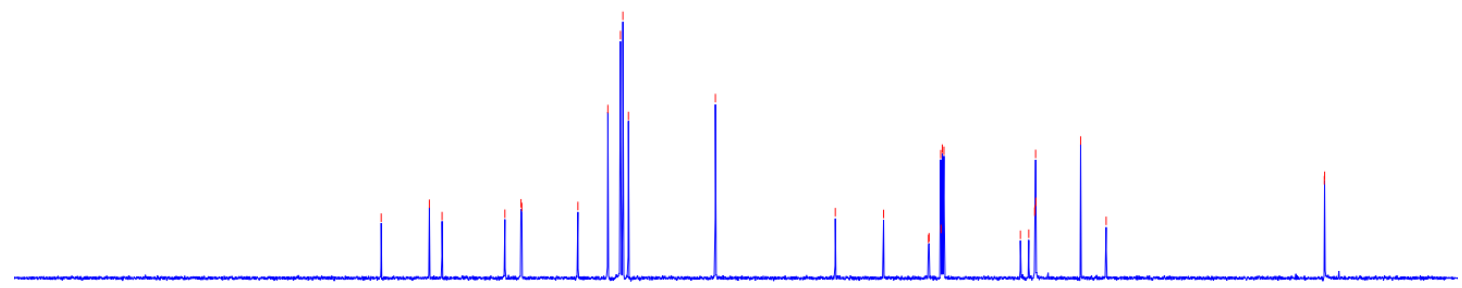

$\begin{array}{lllllllllllllllllllllllll}1 & 1 & 210 & 200 & 190 & 180 & 170 & 160 & 150 & 140 & 130 & 120 & 110 & 100 & 90 & 80 & 70 & 60 & 50 & 40 & 30 & 20 & 10 & 0\end{array}$ 


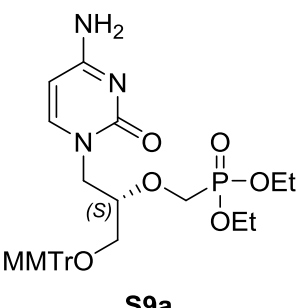

S9a

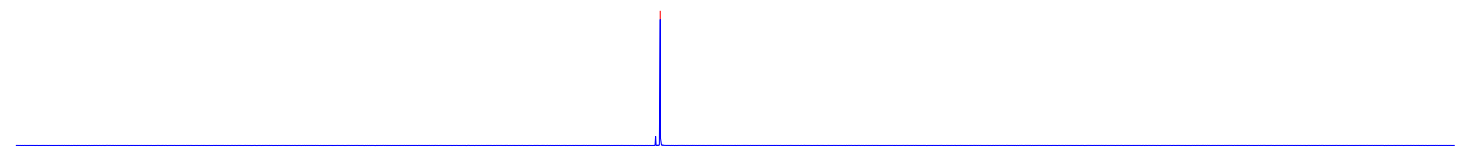

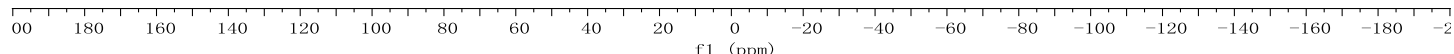

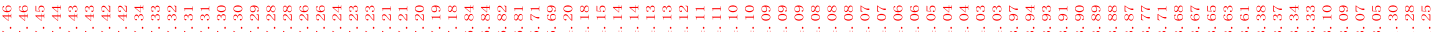
Solvent $\mathrm{CDCl} 3$

Nucleus 1H<smiles>CCOP(=O)(OCC)OCC(COC(C)=O)Cn1ccc(N)nc1=O</smiles>

S9b

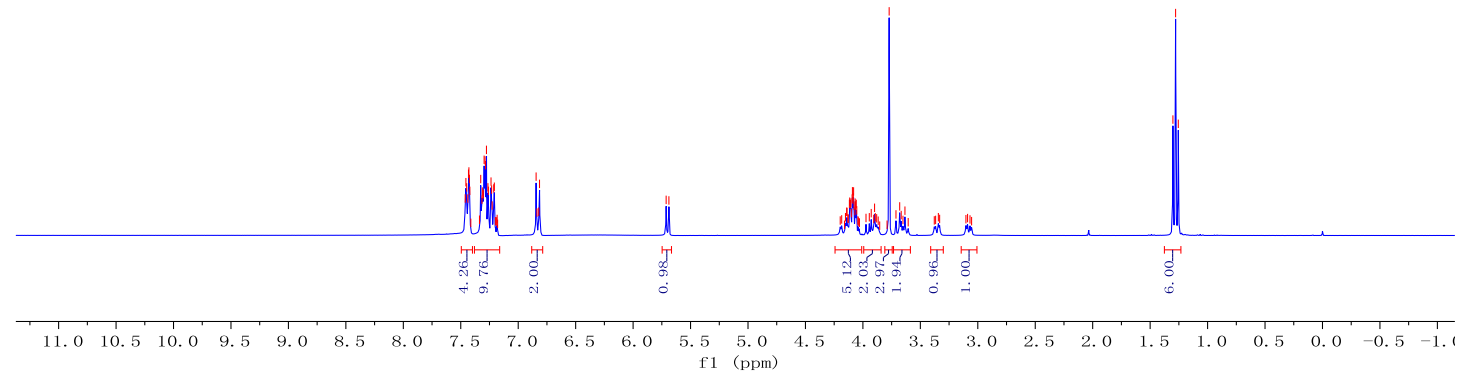




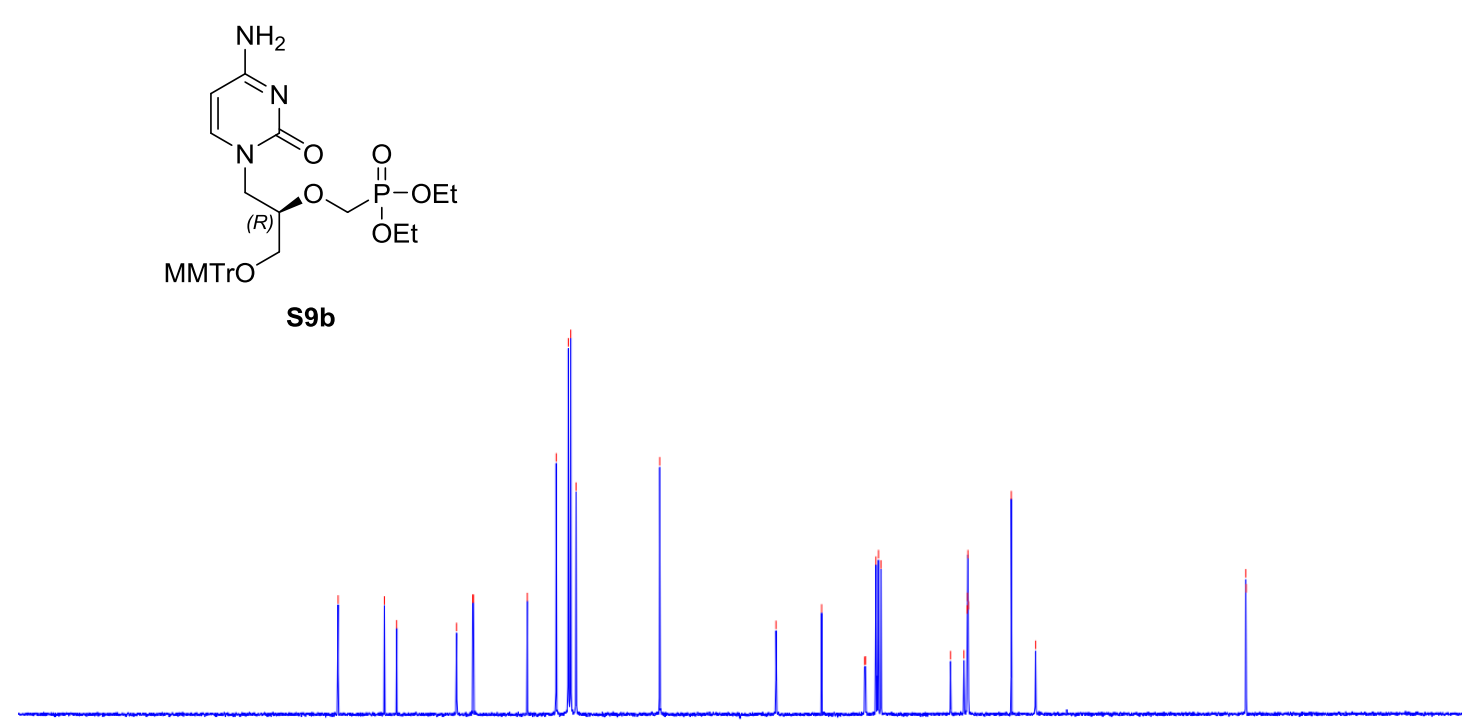

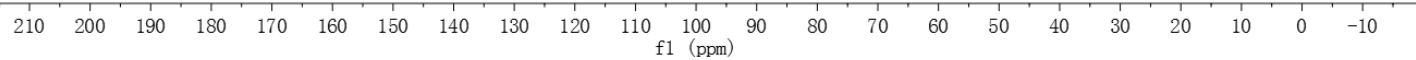

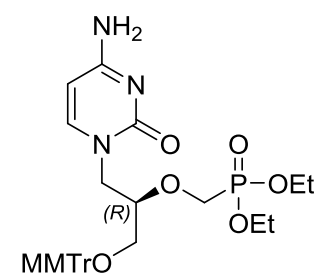

s9b

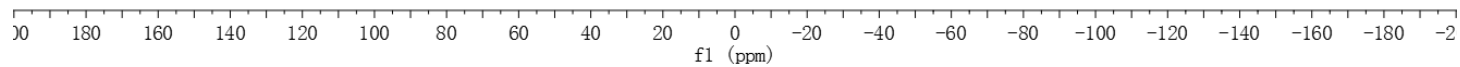




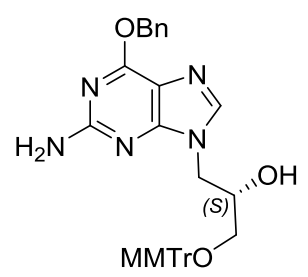

S11a

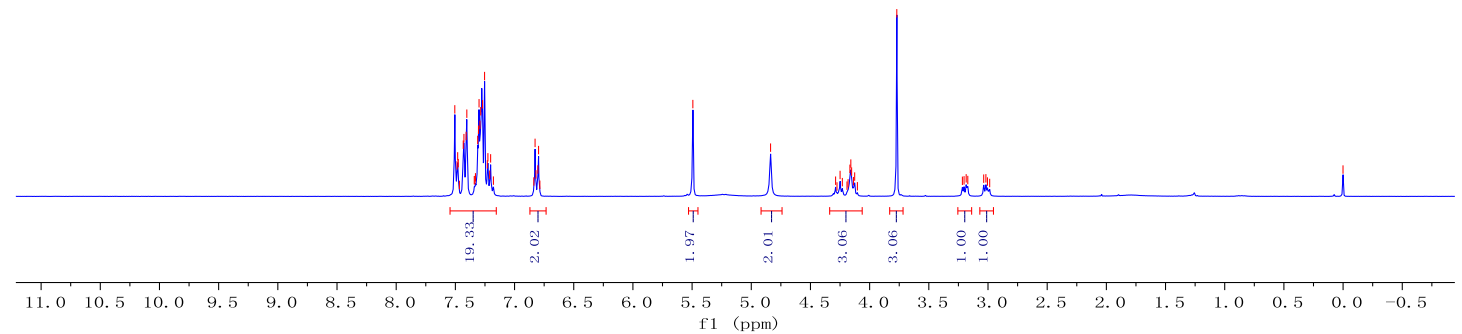

Solvent $\mathrm{CDCl} 3$

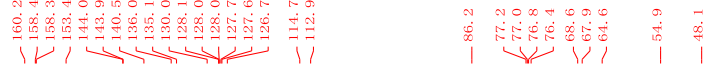

Nucleus 13C

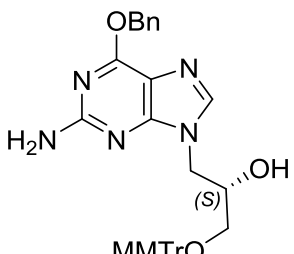

S11a

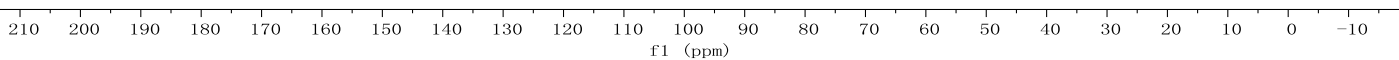




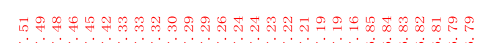
S Solvent CDC
Nucleus $1 \mathrm{H}$

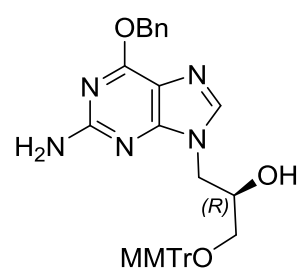

S11b

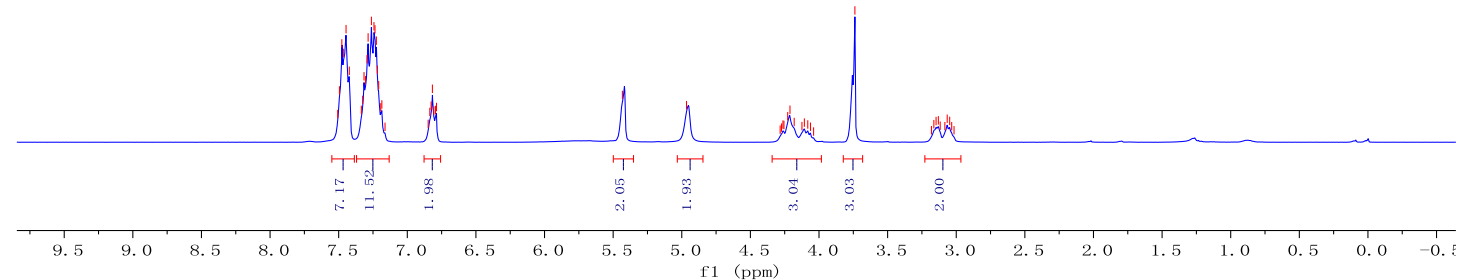

Solvent $\mathrm{CDCl} 3$

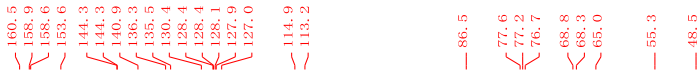

Nucleus 13C

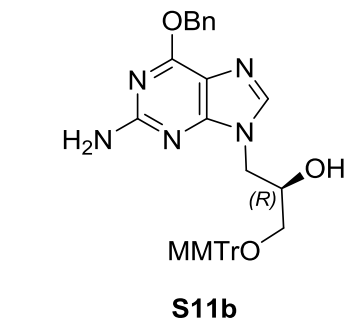

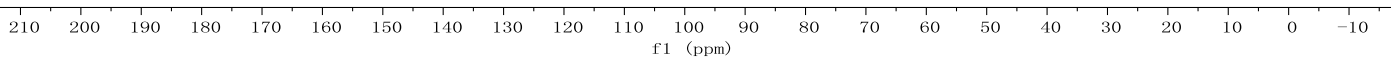




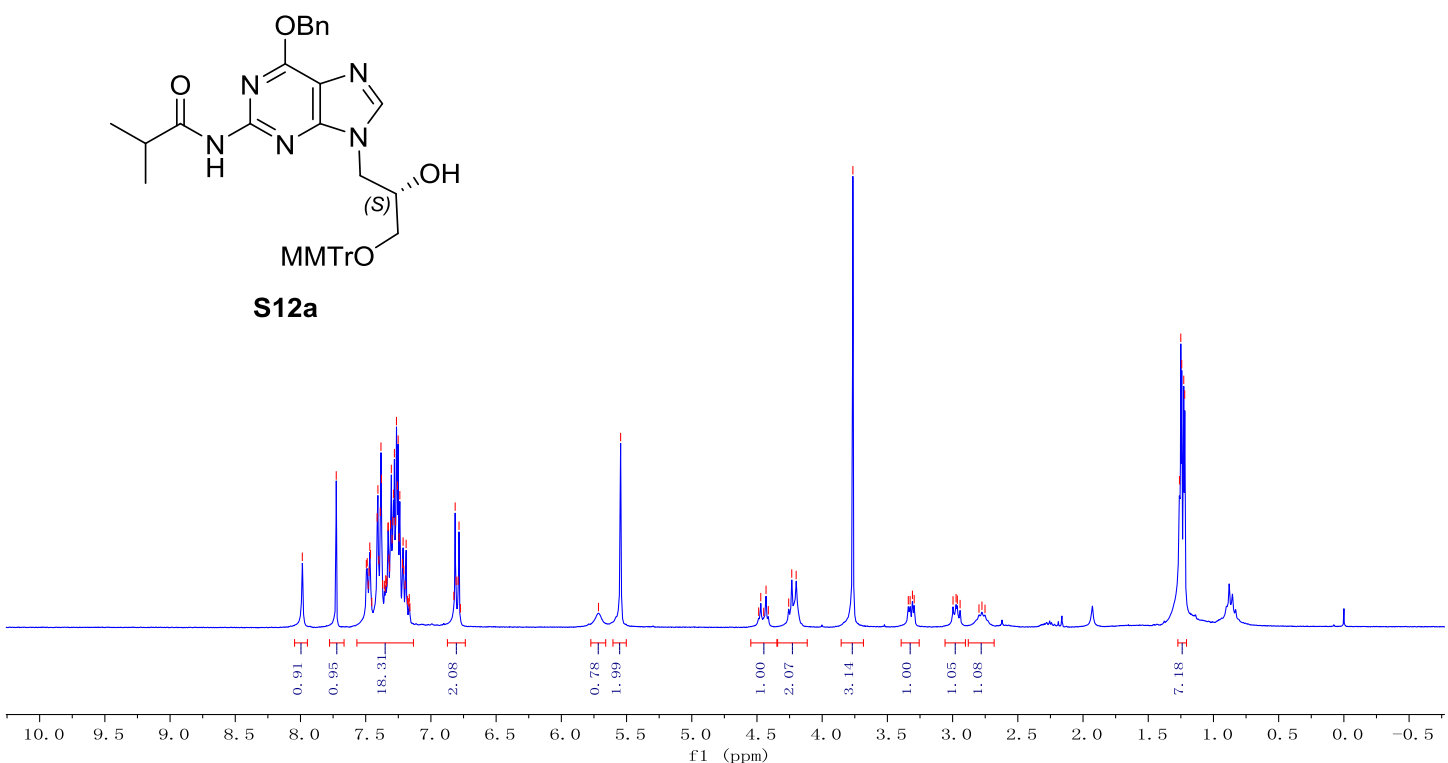

Solvent $\mathrm{CDCl} 3$

Nucleus 13C

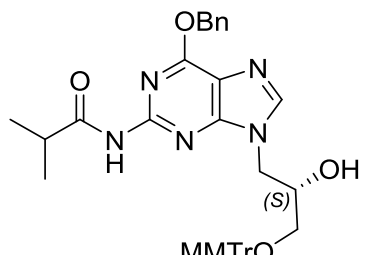

S12a

$\begin{array}{llllllllllllllllllllllllll}1 & 1 \\ 210 & 200 & 190 & 180 & 170 & 160 & 150 & 140 & 130 & 120 & 110 & 100 & 90 & 80 & 70 & 60 & 50 & 40 & 30 & 20 & 10 & 0 & -10\end{array}$ 


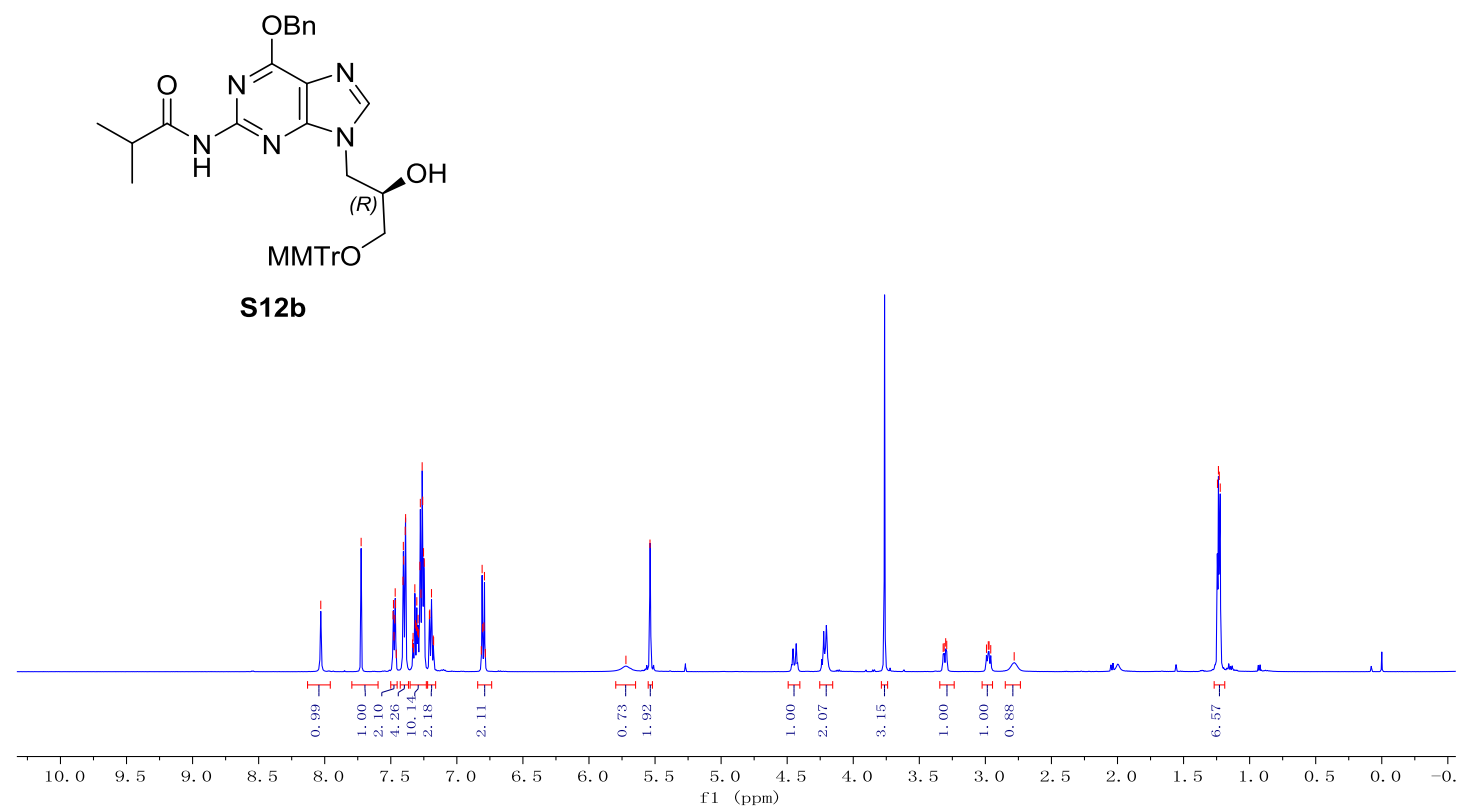

Solvent $\mathrm{CDCl} 3$

Nucleus 13C

$1 / 1 / V^{\prime}$

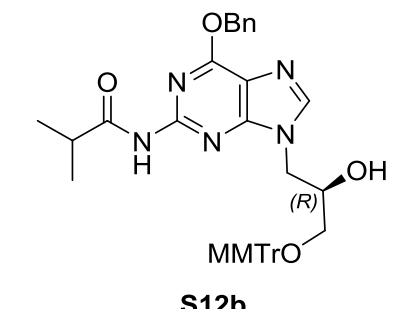

$\mathrm{S} 12 \mathrm{~b}$ 


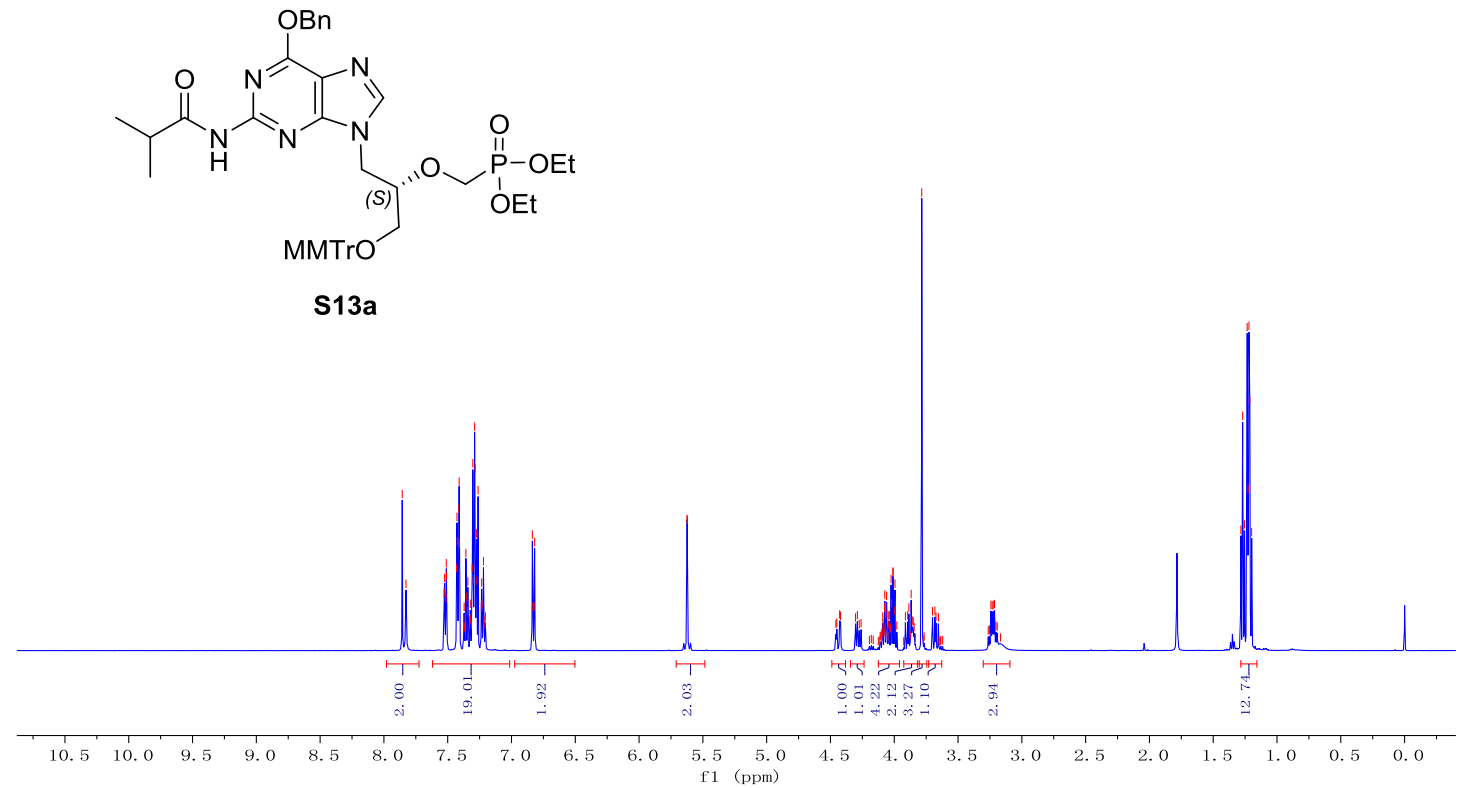

Solvent $\mathrm{CDCl} 3$

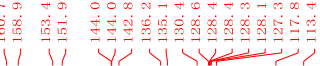

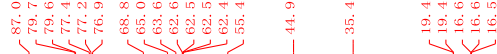

Nucleus 13C

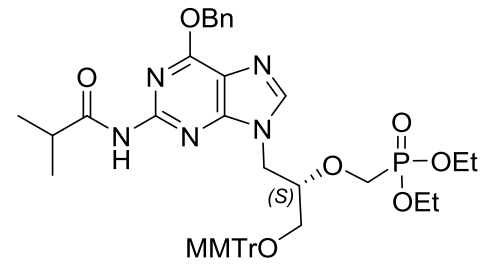

S13a 


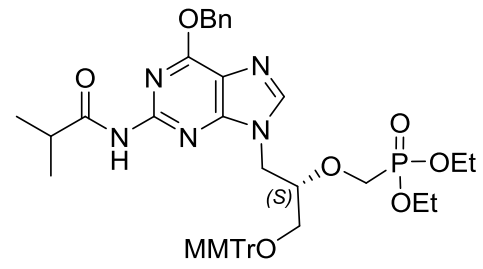

S13a

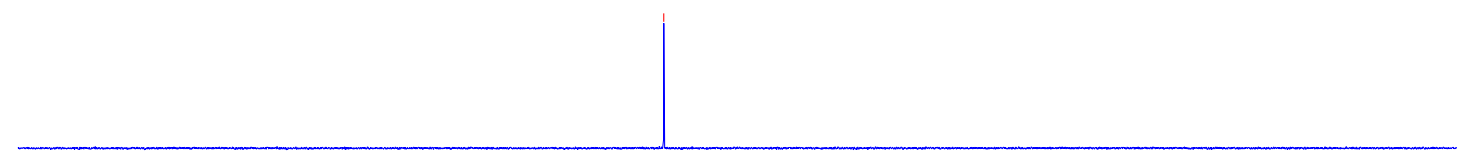

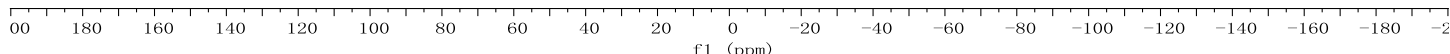

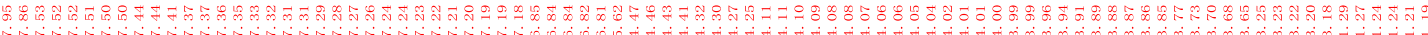
Solvent $\mathrm{CDCl} 3$

Nucleus 1H
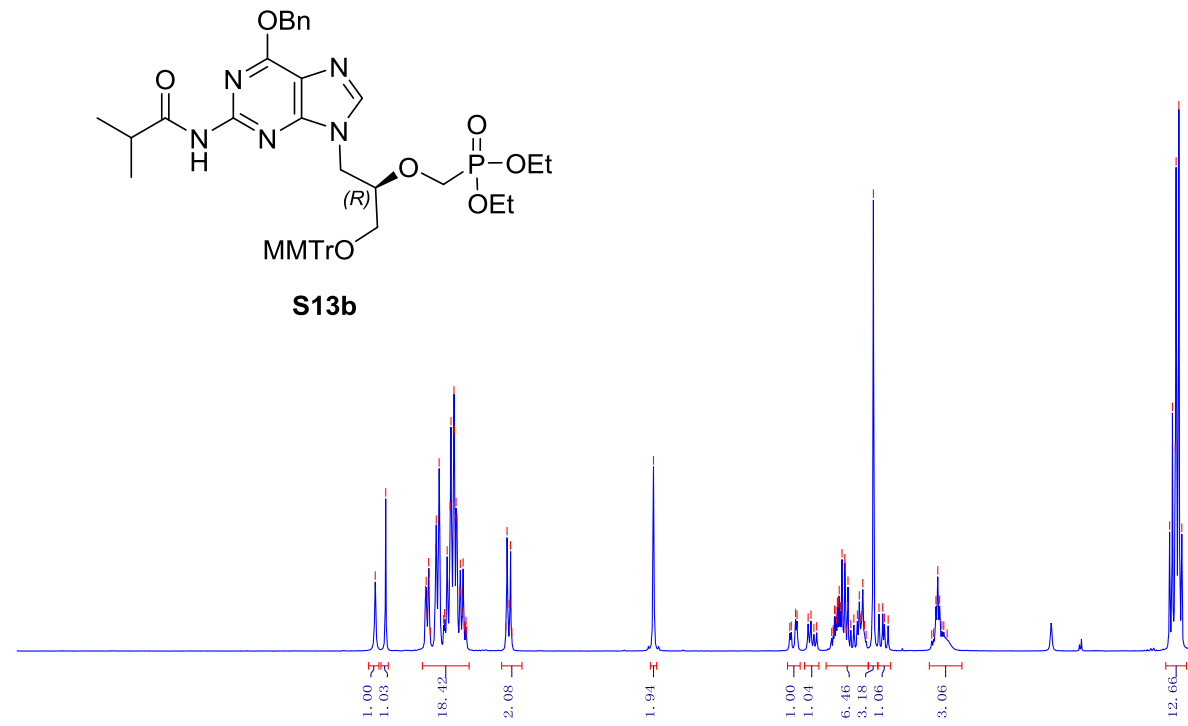

$\begin{array}{lllllllllllllllllllllllllllllllllllllllll}10.5 & 10.0 & 9.5 & 9.0 & 8.5 & 8.0 & 7.5 & 7.0 & 6.5 & 6.0 & 5.5 & 5.0 & 4.5 & 4.0 & 3.5 & 3.0 & 2.5 & 2.0 & 1.5 & 1.0 & 0.5 & 0.0 & -0.5 & -1 .\end{array}$ 


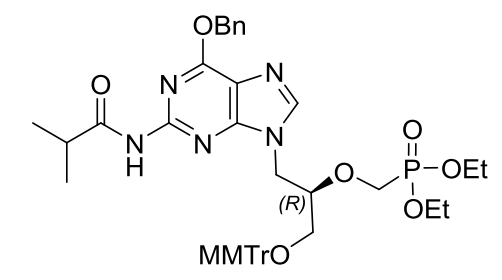

S13b
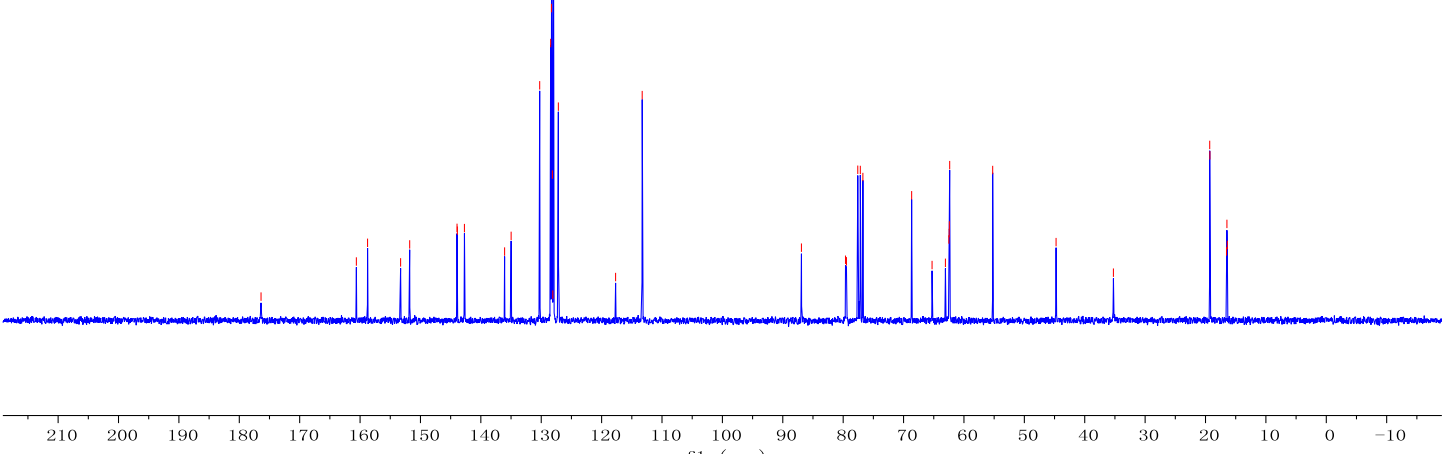

Solvent $\mathrm{CDCl} 3$

Nucleus 31P

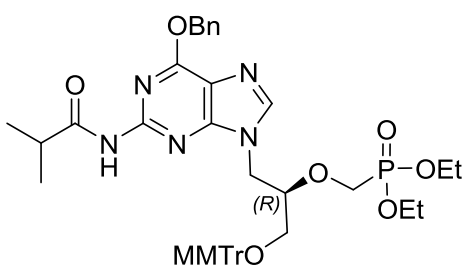

S13b

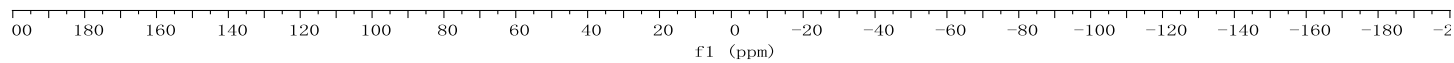




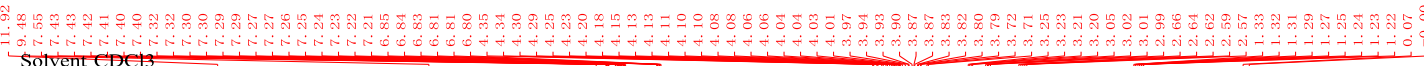
Solvent CDE

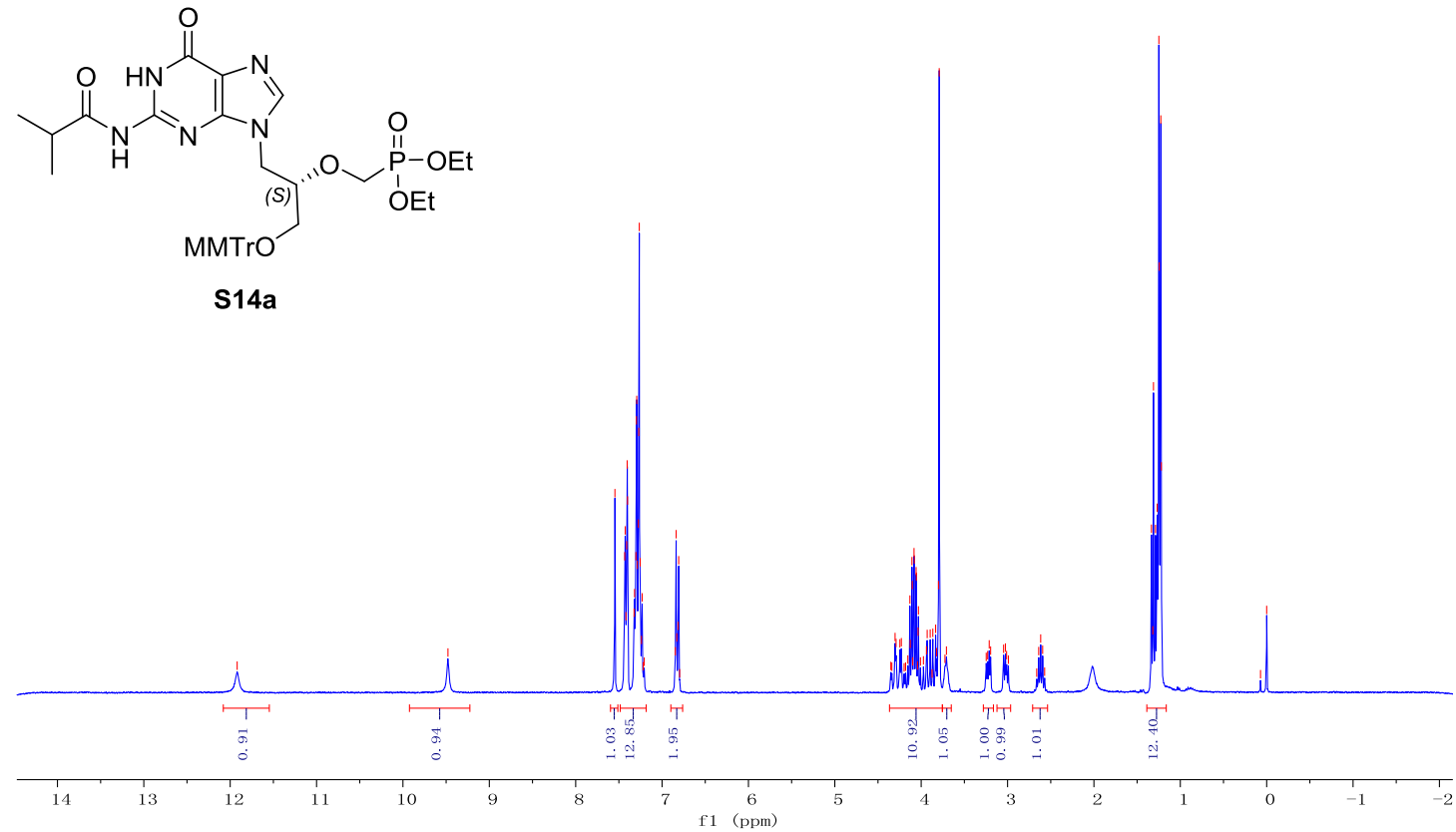

Solvent $\mathrm{CDCl} 3$

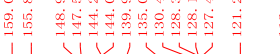

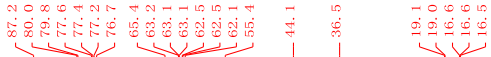

Nucleus 13C

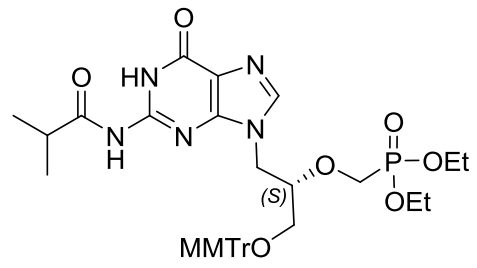

S14a

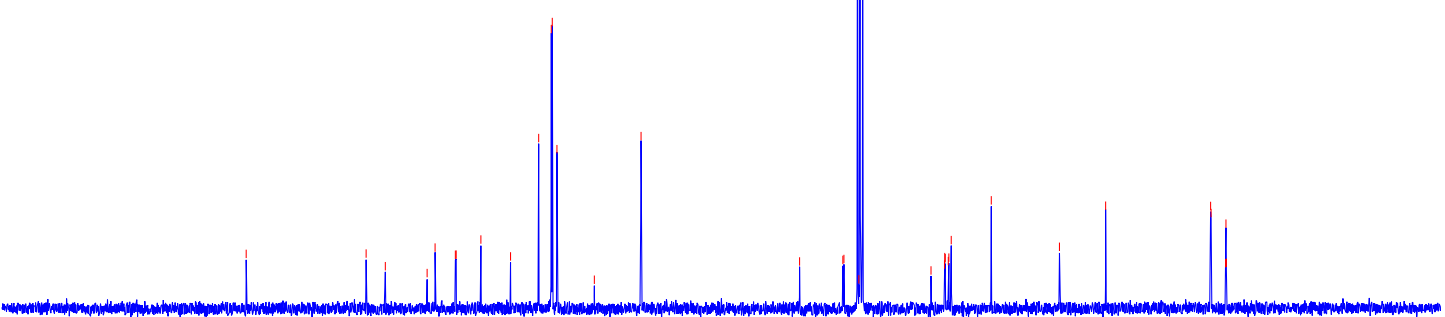

$\begin{array}{llllllllllllllllllllllllll}210 & 200 & 190 & 180 & 170 & 160 & 150 & 140 & 130 & 120 & 110 & 100 & 90 & 80 & 70 & 60 & 50 & 40 & 30 & 20 & 10 & 0 & -10\end{array}$ 


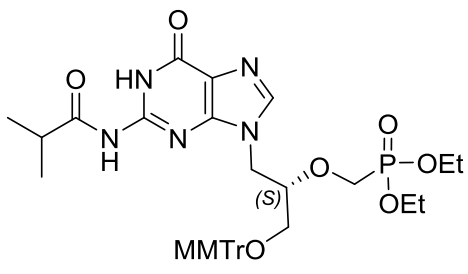

S14a

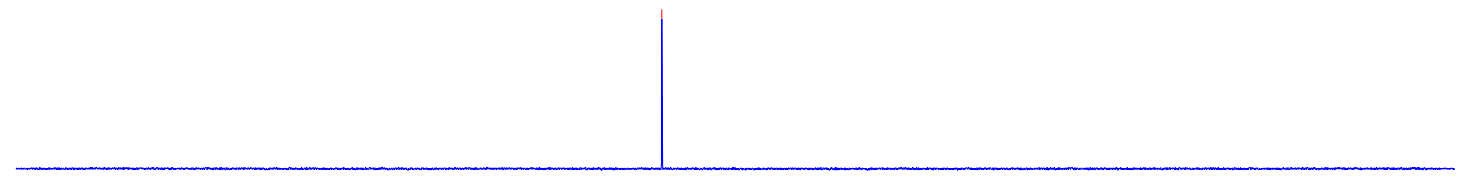

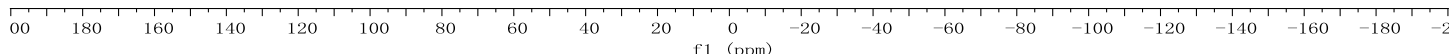

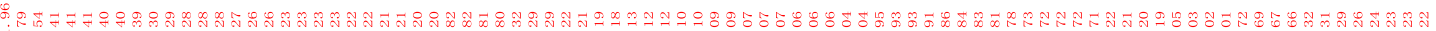
Solvent Nucleus $1 \mathrm{H}$

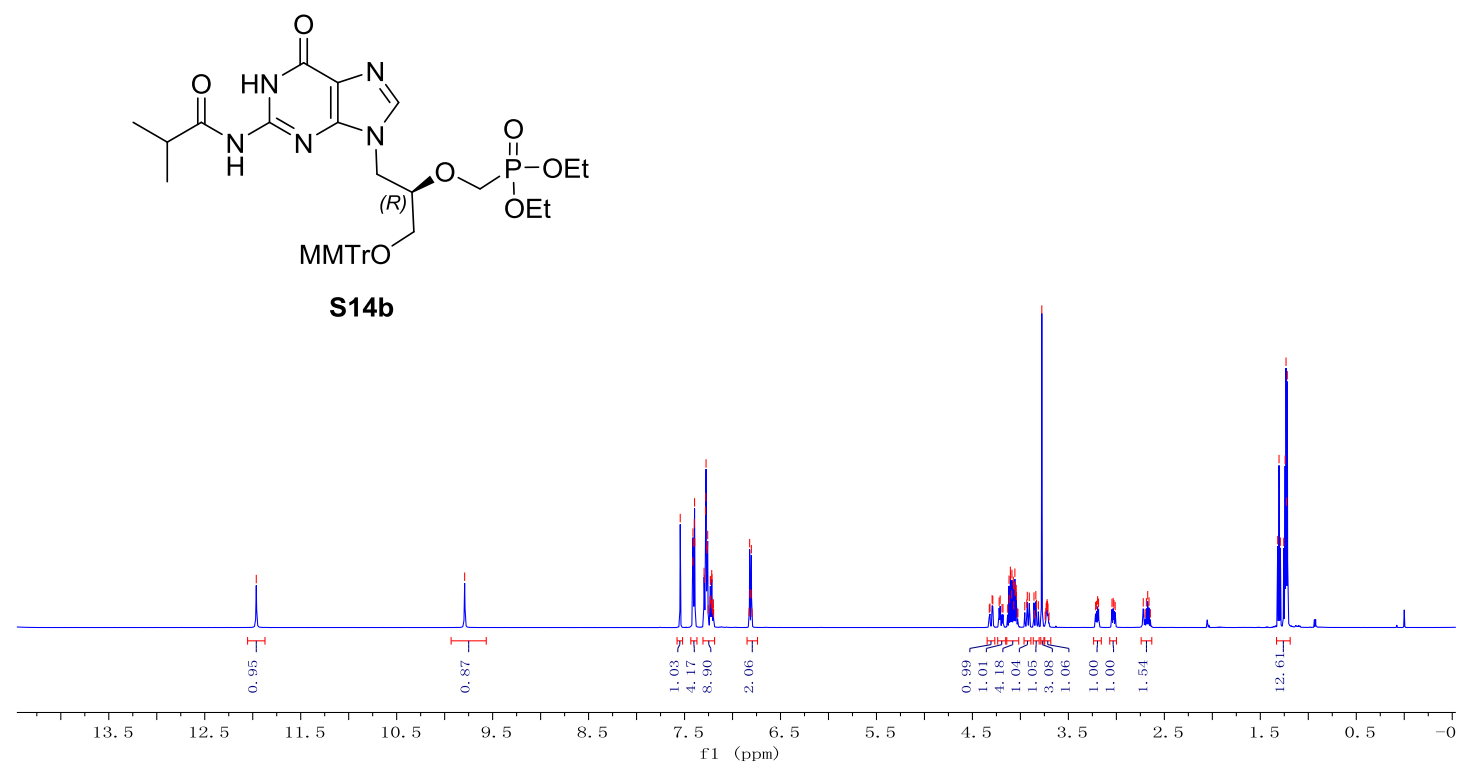




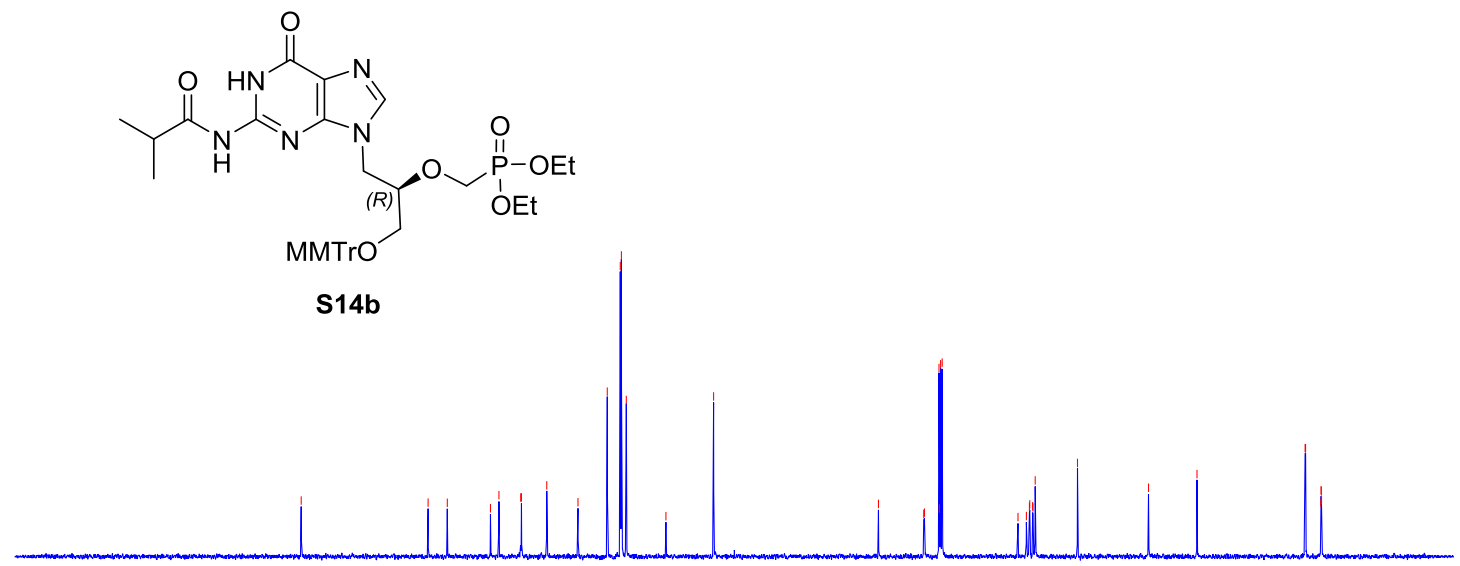

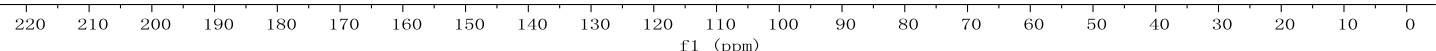

Solvent $\mathrm{CDCl} 3$

Nucleus 31P

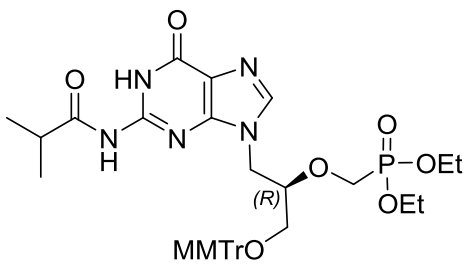

S14b

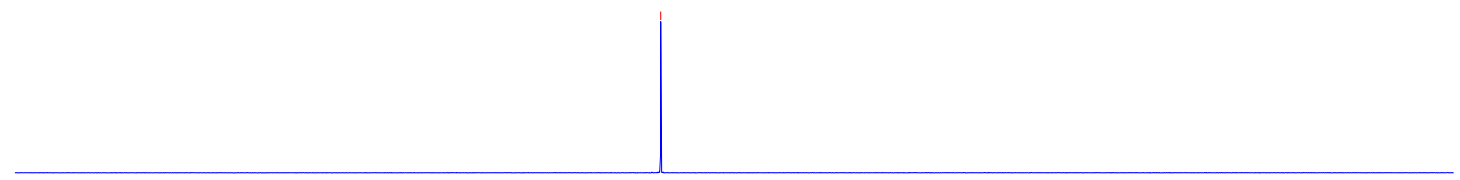

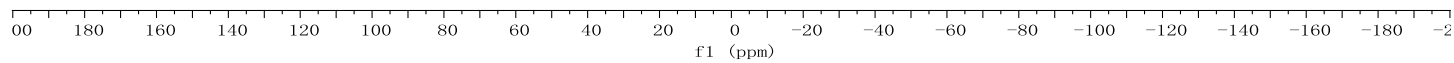




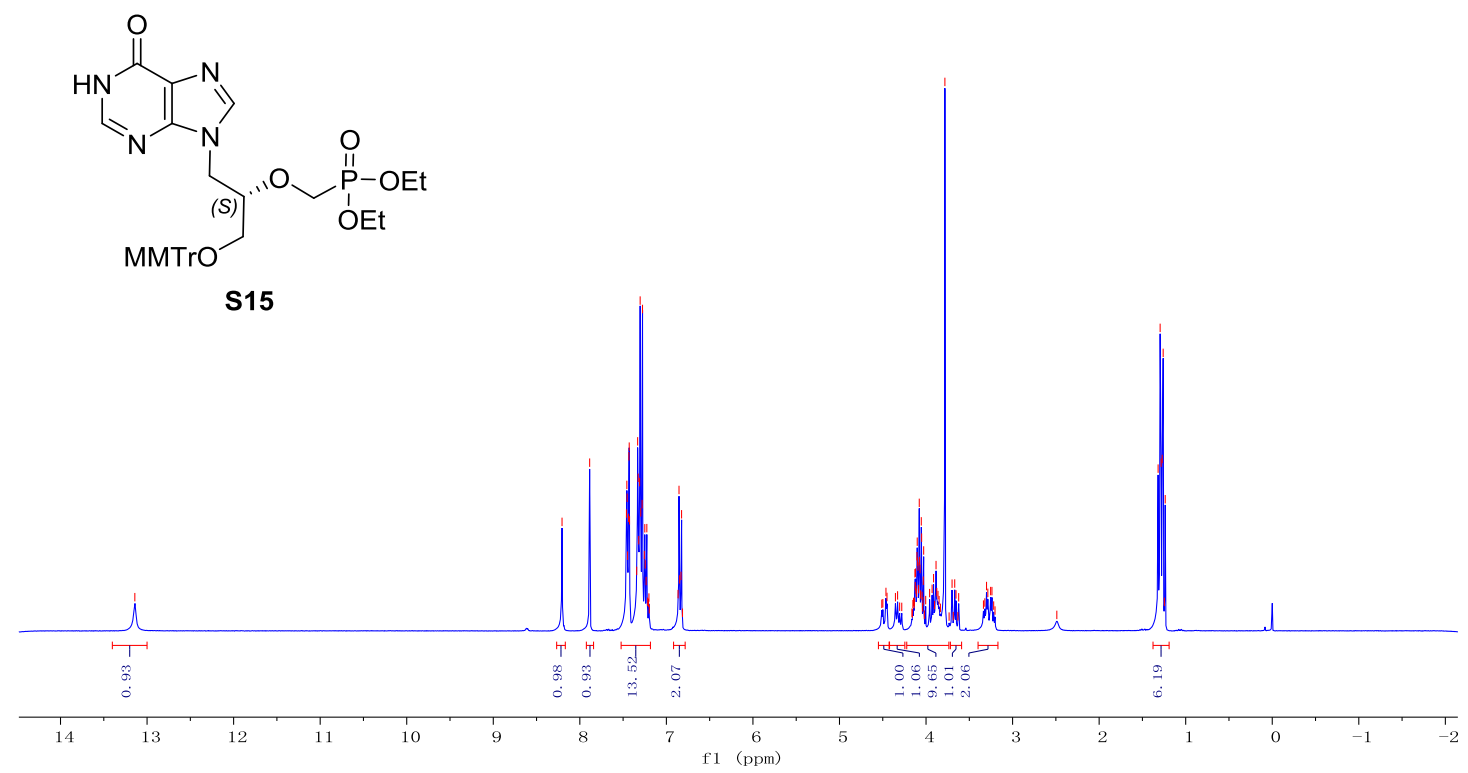

Solvent $\mathrm{CDCl} 3$

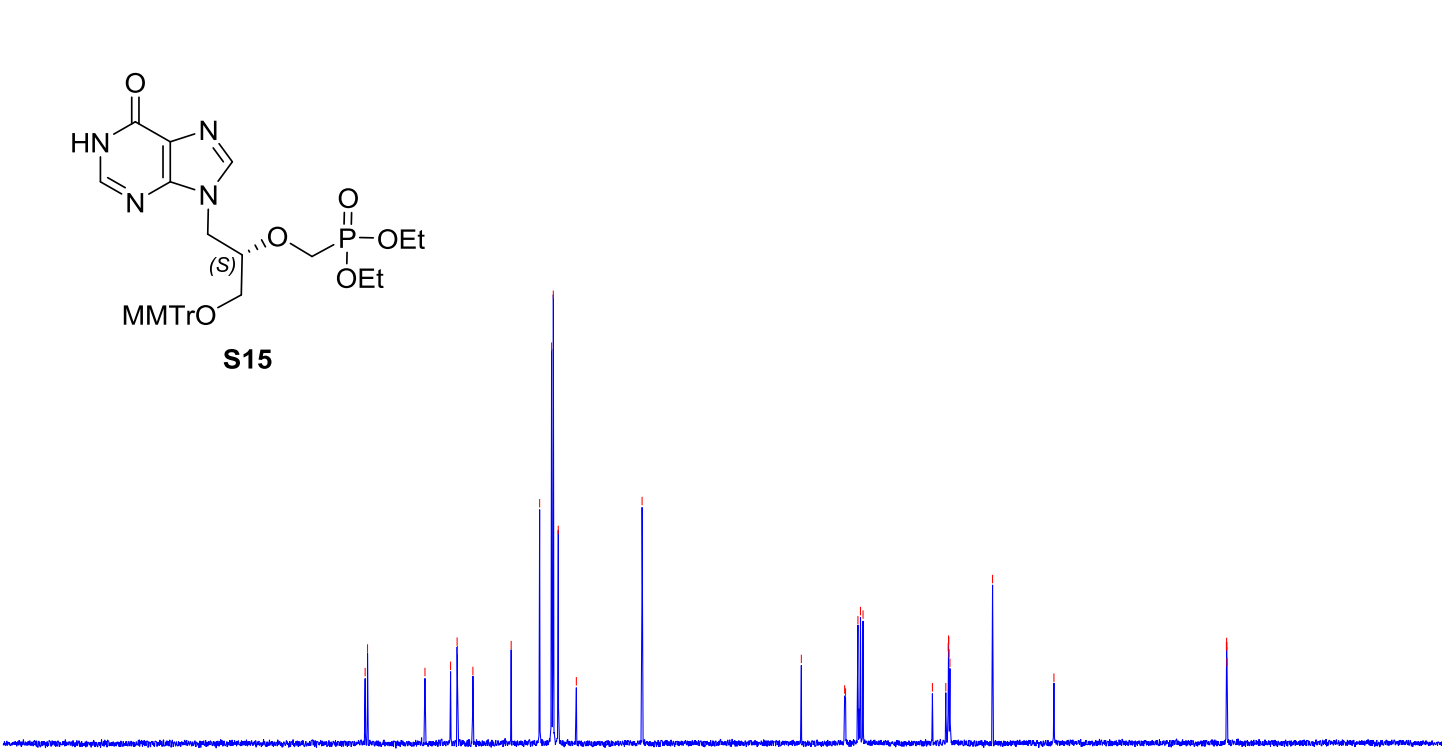

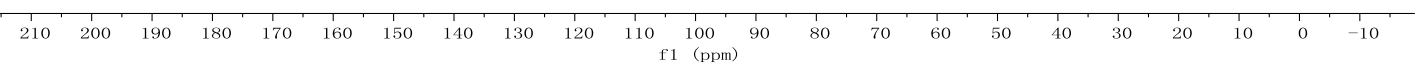



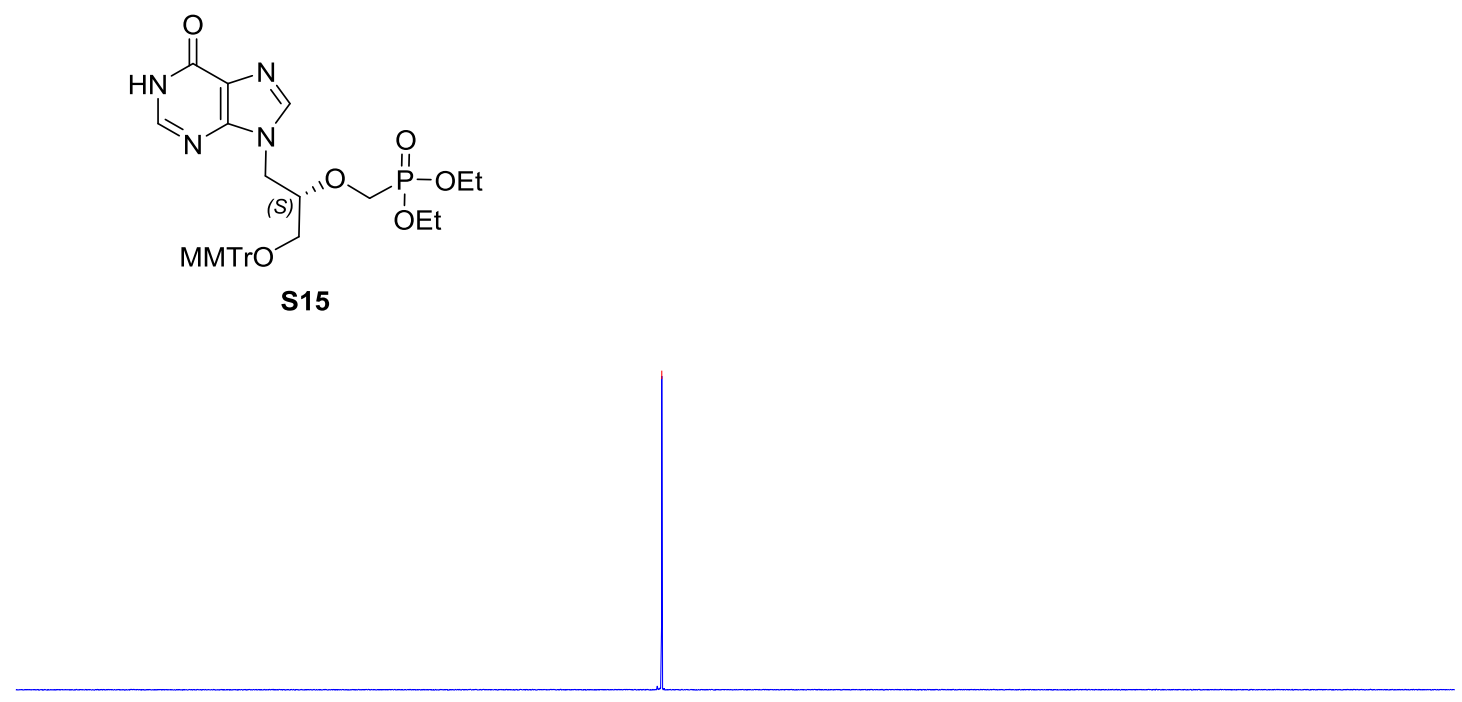

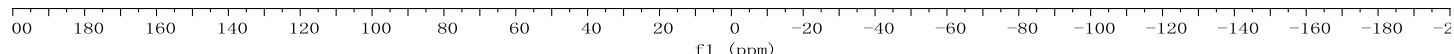

Solvent CD3OD

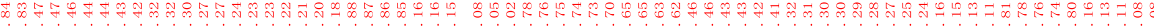
Nucleus $1 \mathrm{H}$

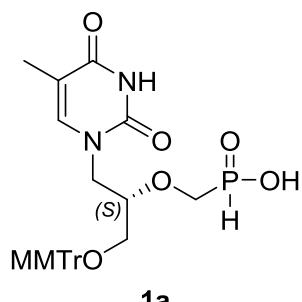

$1 \mathbf{a}$

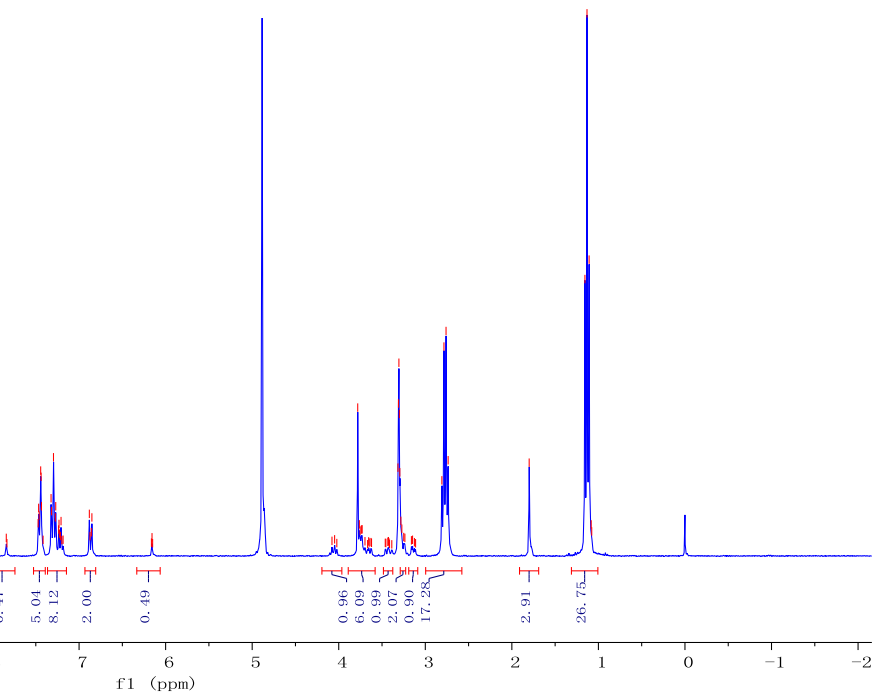




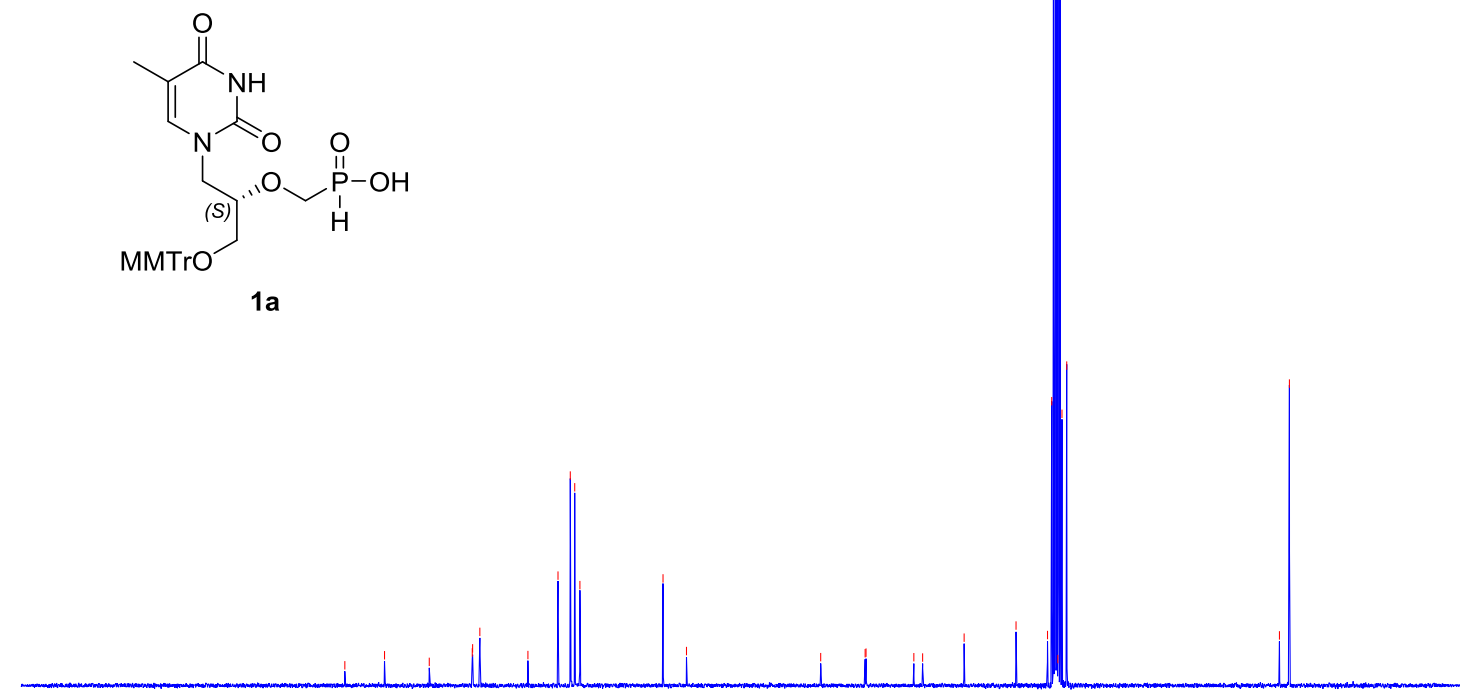

\section{| V|}

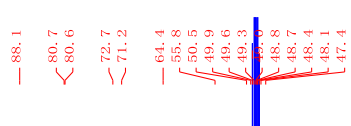

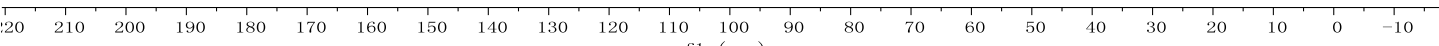

Solvent CD3OD

Nucleus 31P

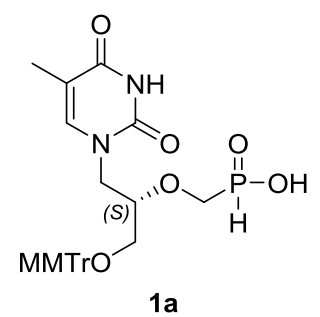

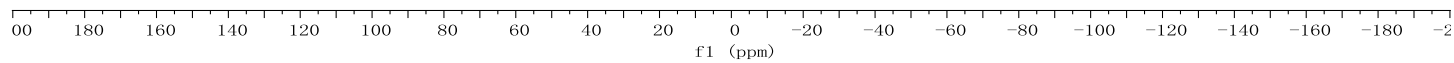


<smiles>COCC(COP(=O)(O)O)OCn1cc(C)c(=O)[nH]c1=O</smiles>

$1 b$

Solvent CD3OD

Nucleus 13C

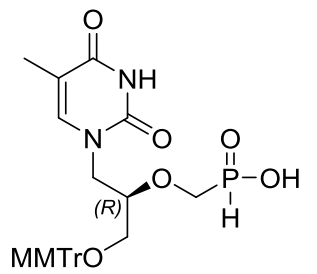

1b

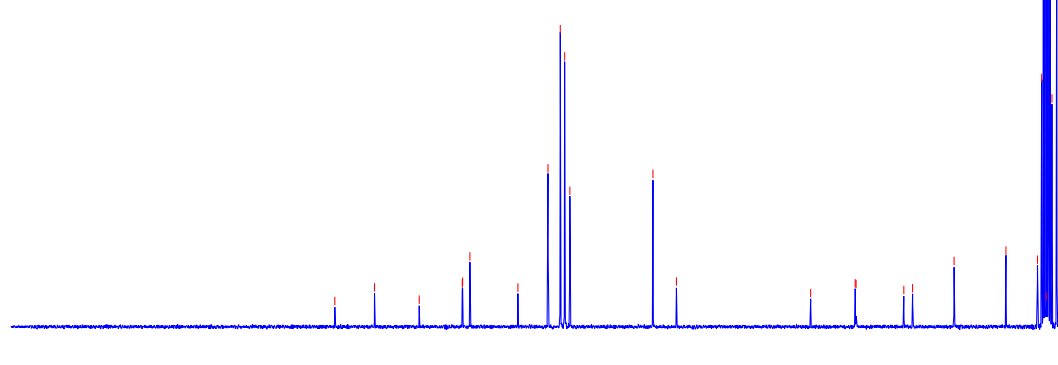

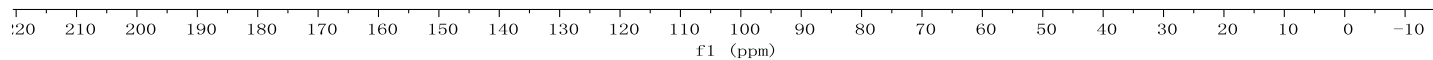




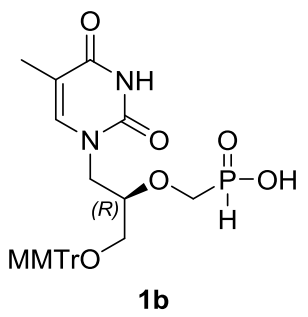

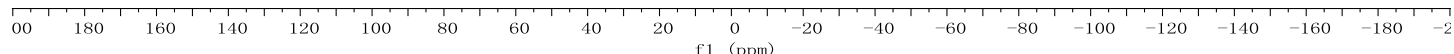

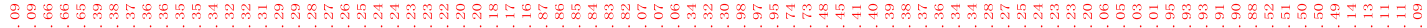
Solvent DMSO-d6

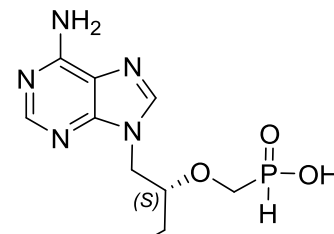

MMTrO

S19a

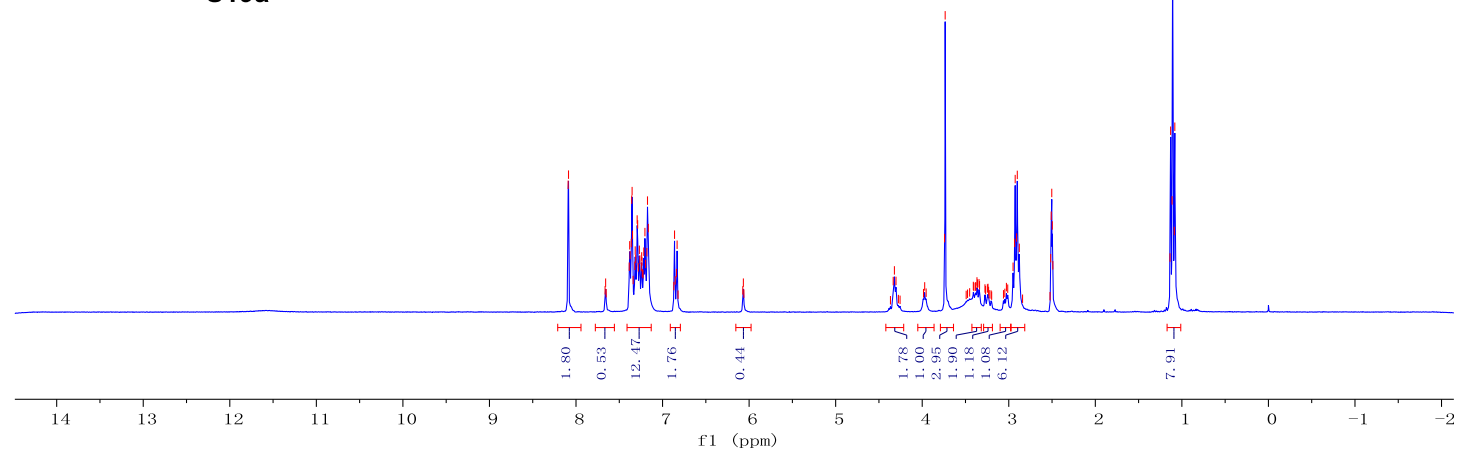


Solvent

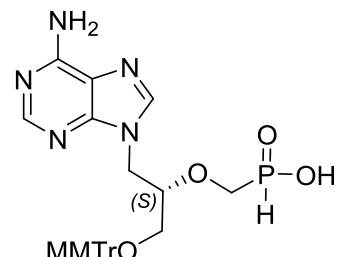

S19a

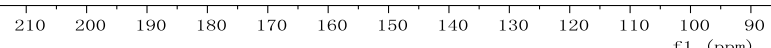

Solvent DMSO-d6

Nucleus 31P

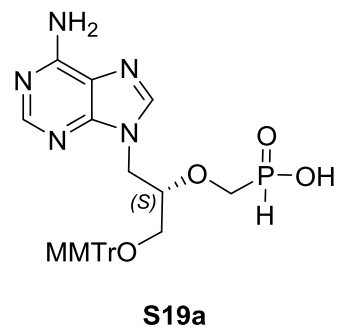

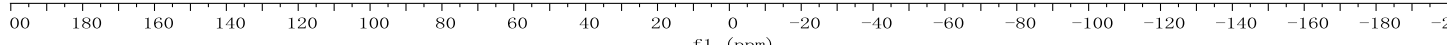




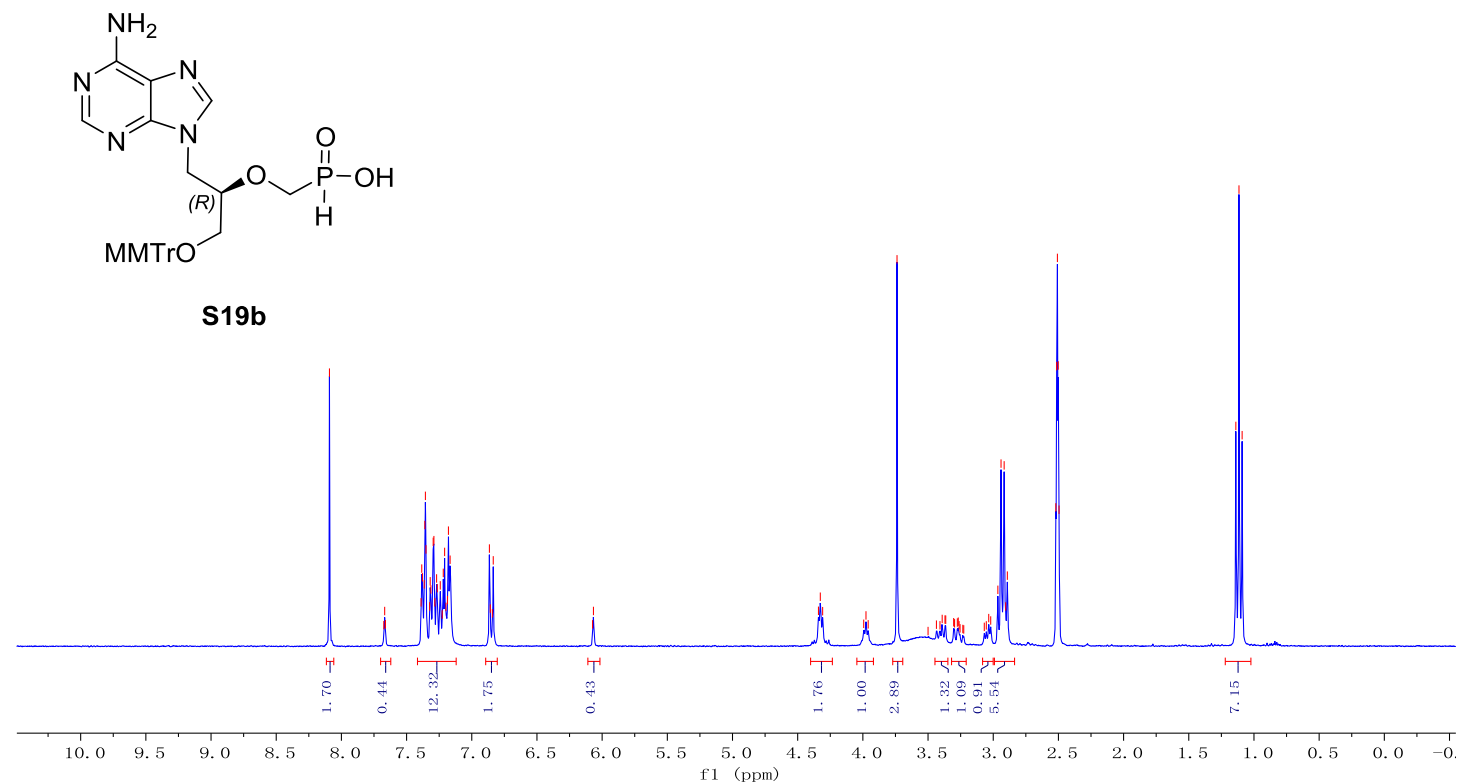

Solvent DMSO- $d 6$

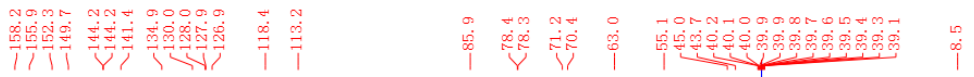

Nucleus 13C

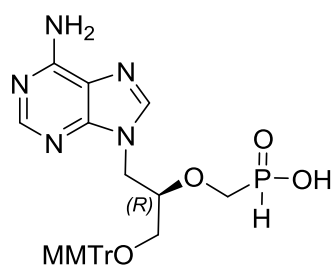

S19b

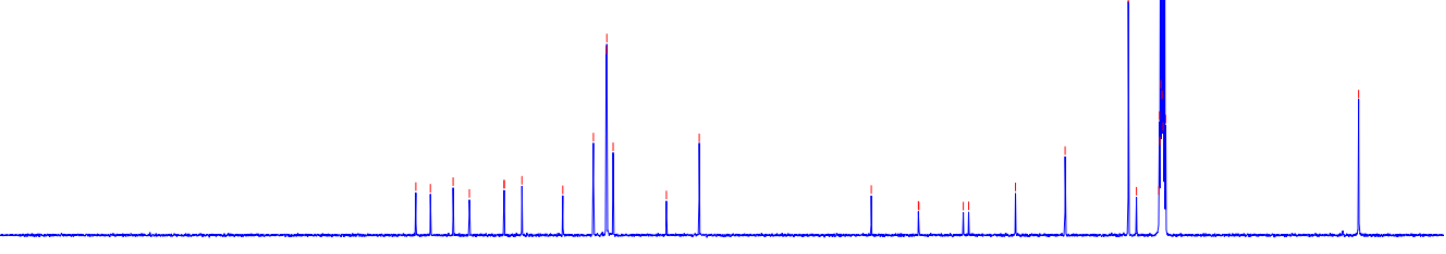

$\begin{array}{lllllllllllllllllllllllll}220 & 210 & 200 & 190 & 180 & 170 & 160 & 150 & 140 & 130 & 120 & 110 & 100 & 90 & 80 & 70 & 60 & 50 & 40 & 30 & 20 & 10 & 0\end{array}$ 
Solvent DMSO-d6

Nucleus $31 \mathrm{P}$
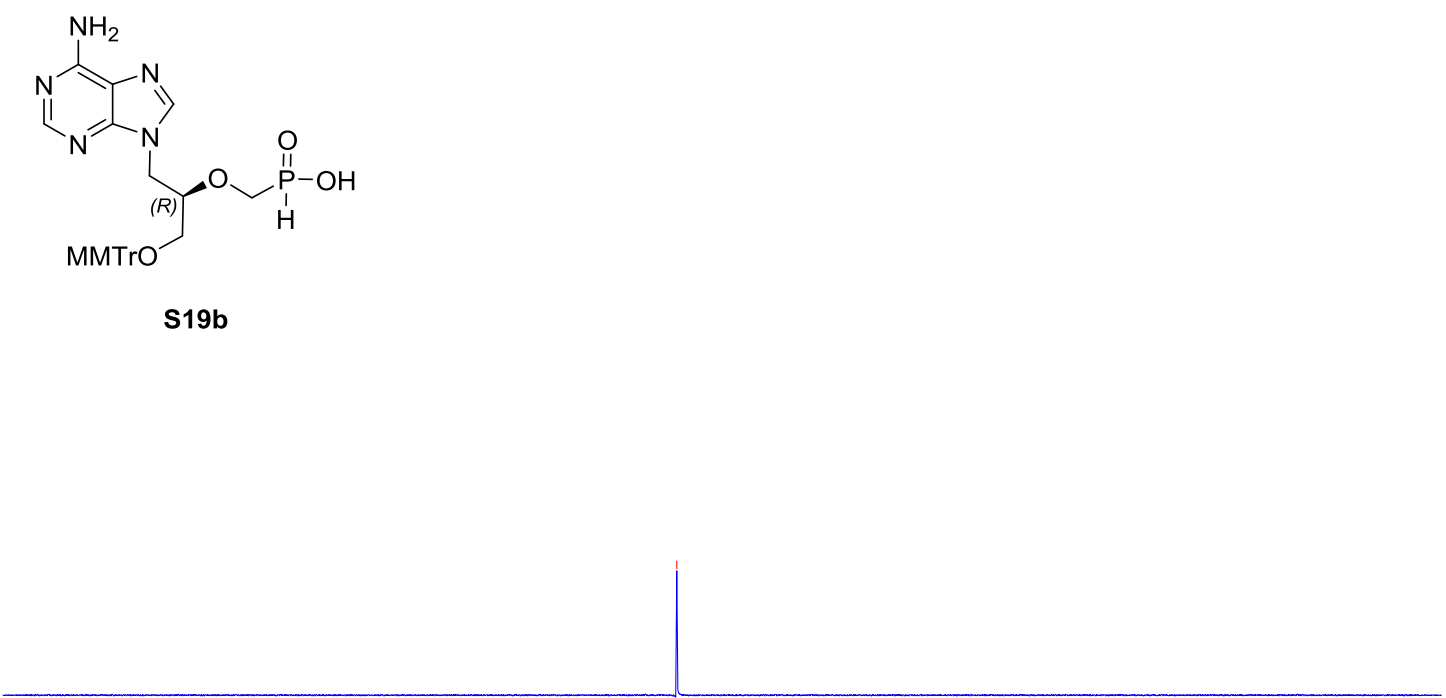

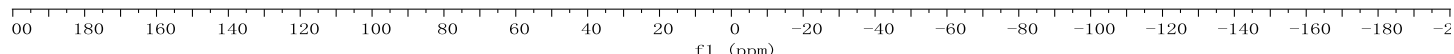

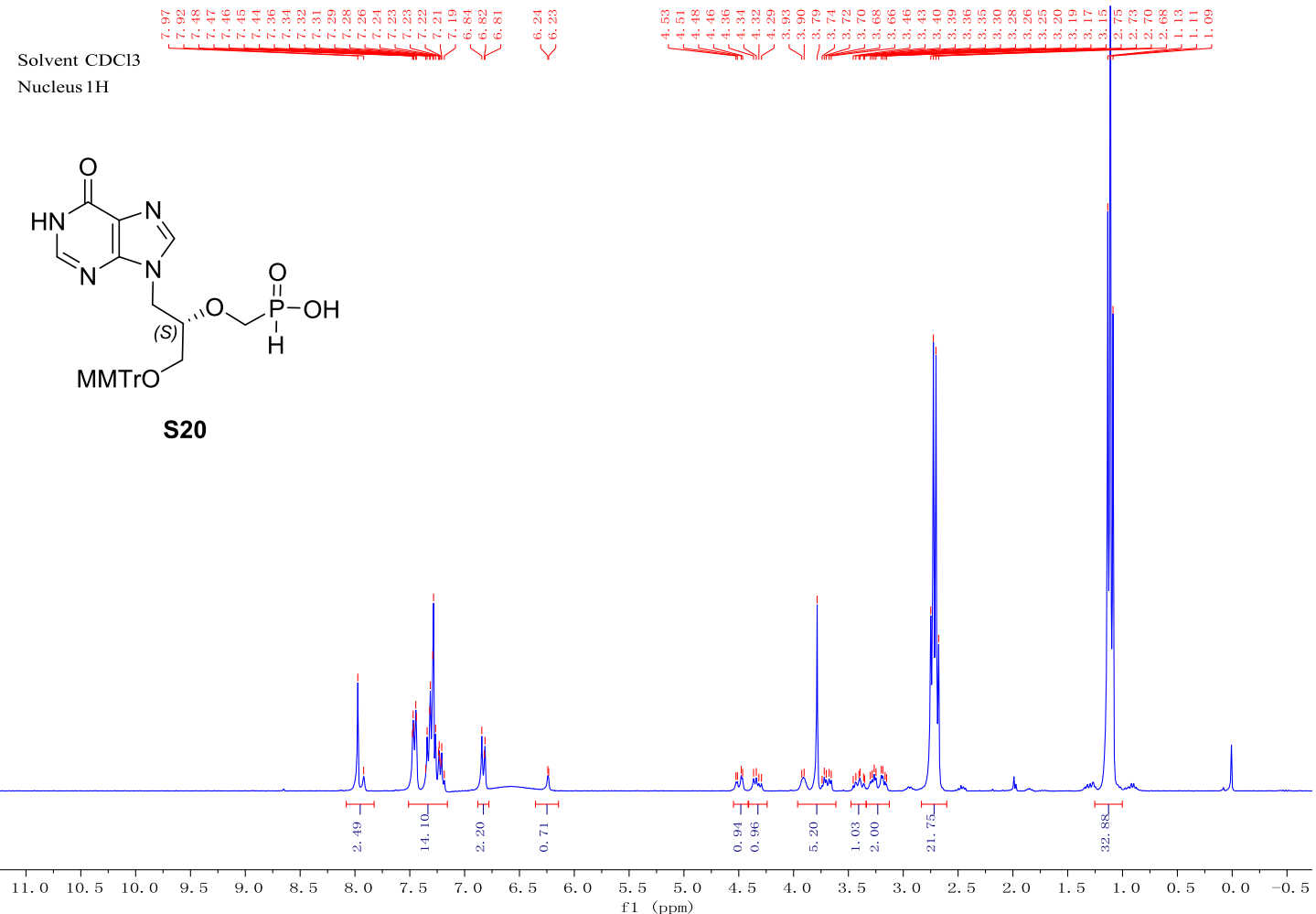




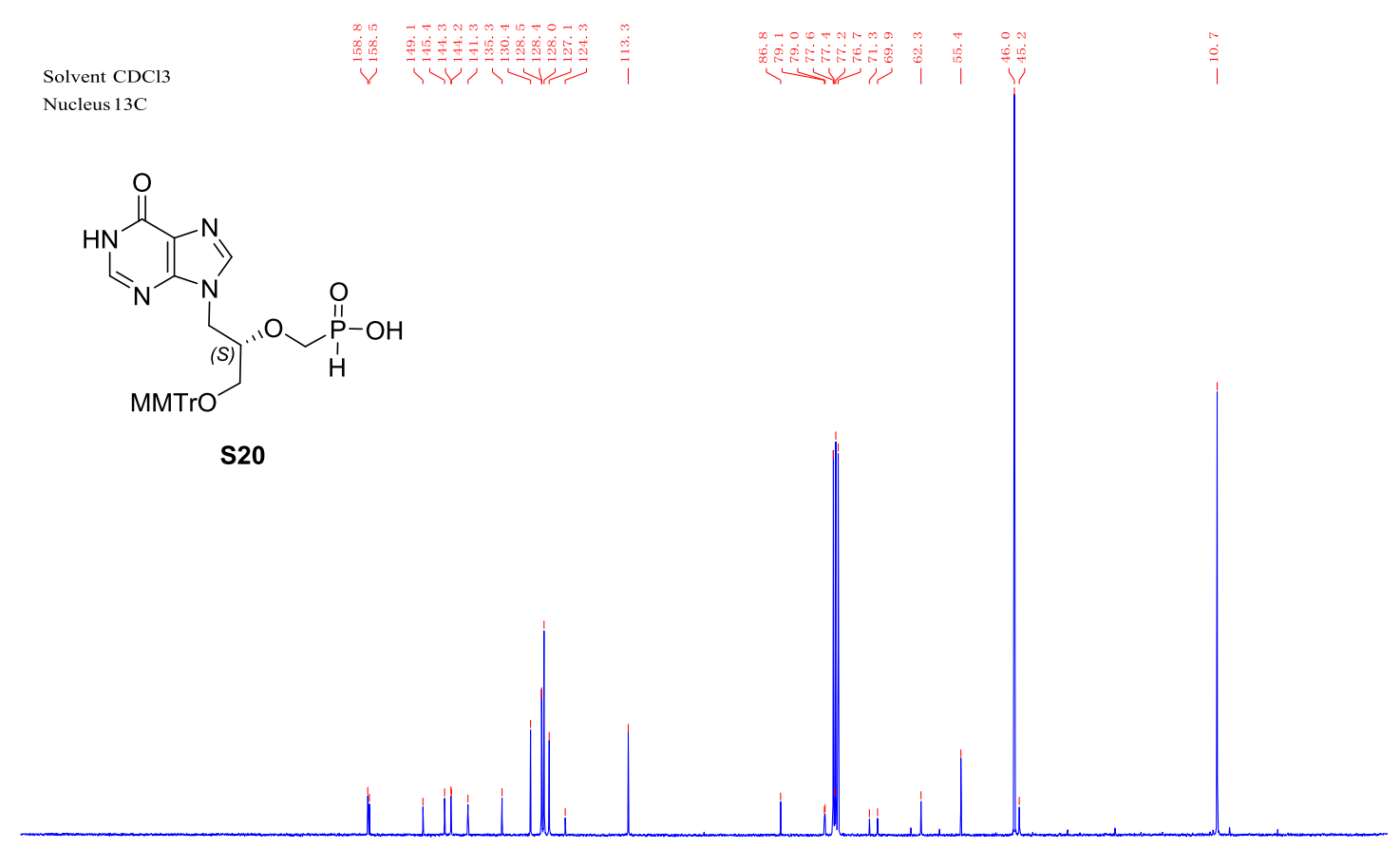

Solvent $\mathrm{CDCl} 3$

Nucleus 31P

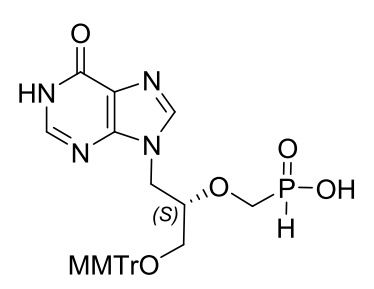

S20

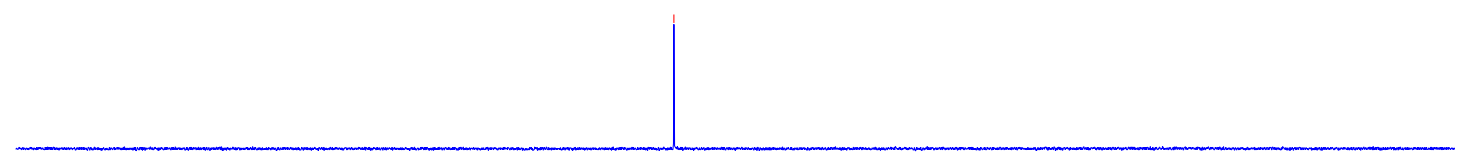

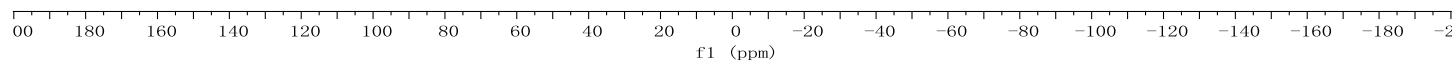




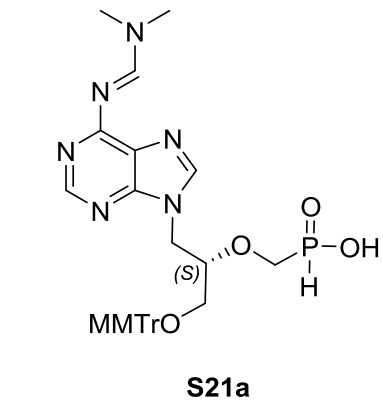

Solvent $\mathrm{CDCl} 3$
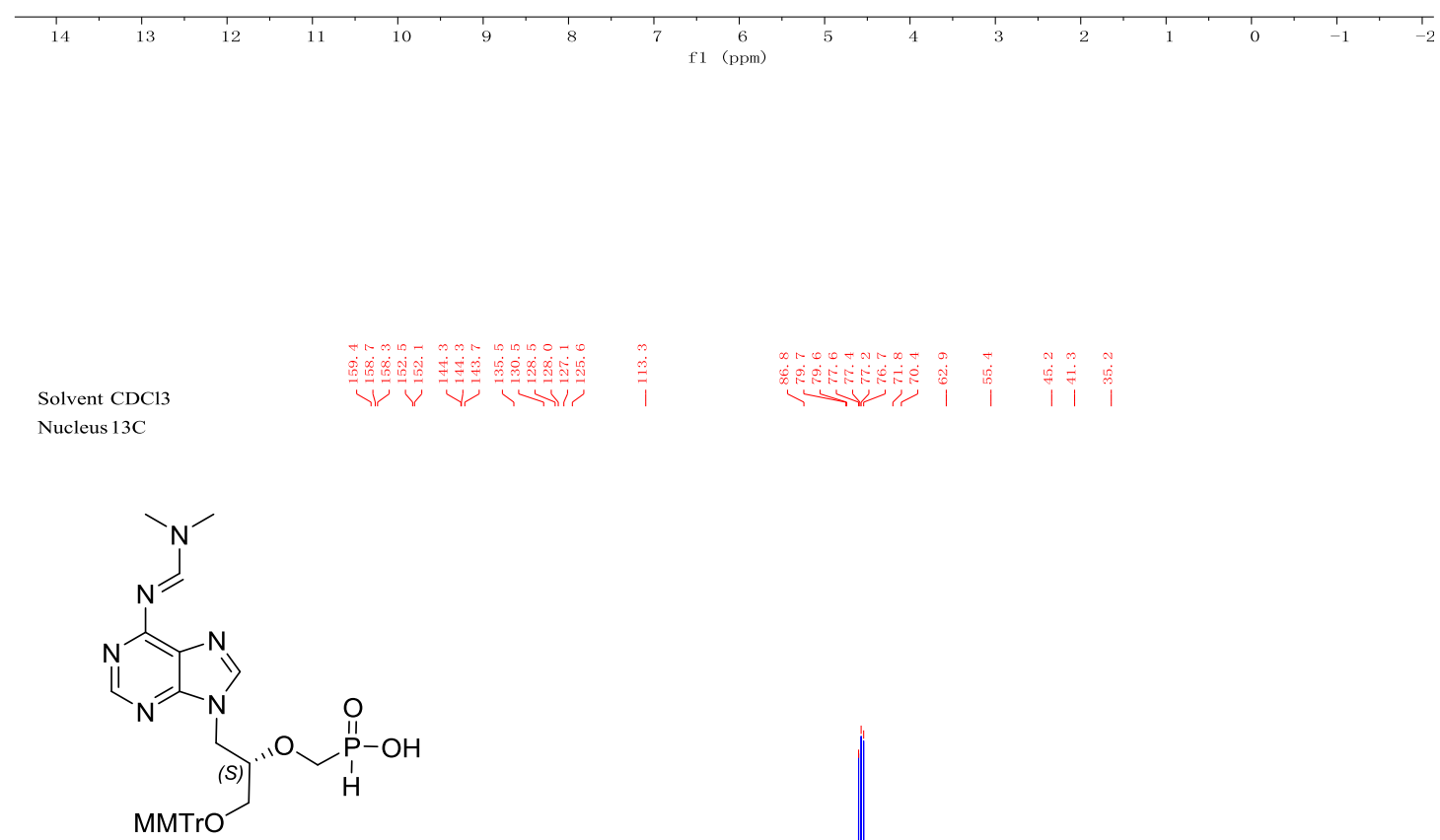

S21a

$\begin{array}{lllllllllllll}210 & 200 & 190 & 180 & 170 & 160 & 150 & 140 & 130 & 120 & 110 & 100 & 90\end{array}$ 


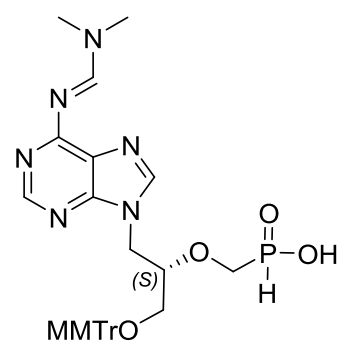

S21a

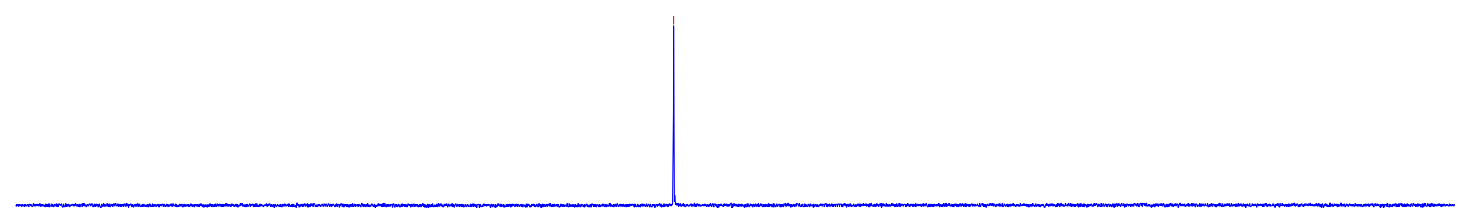

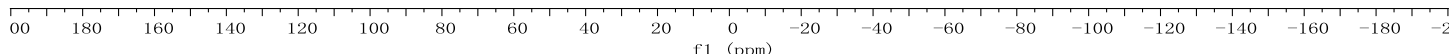

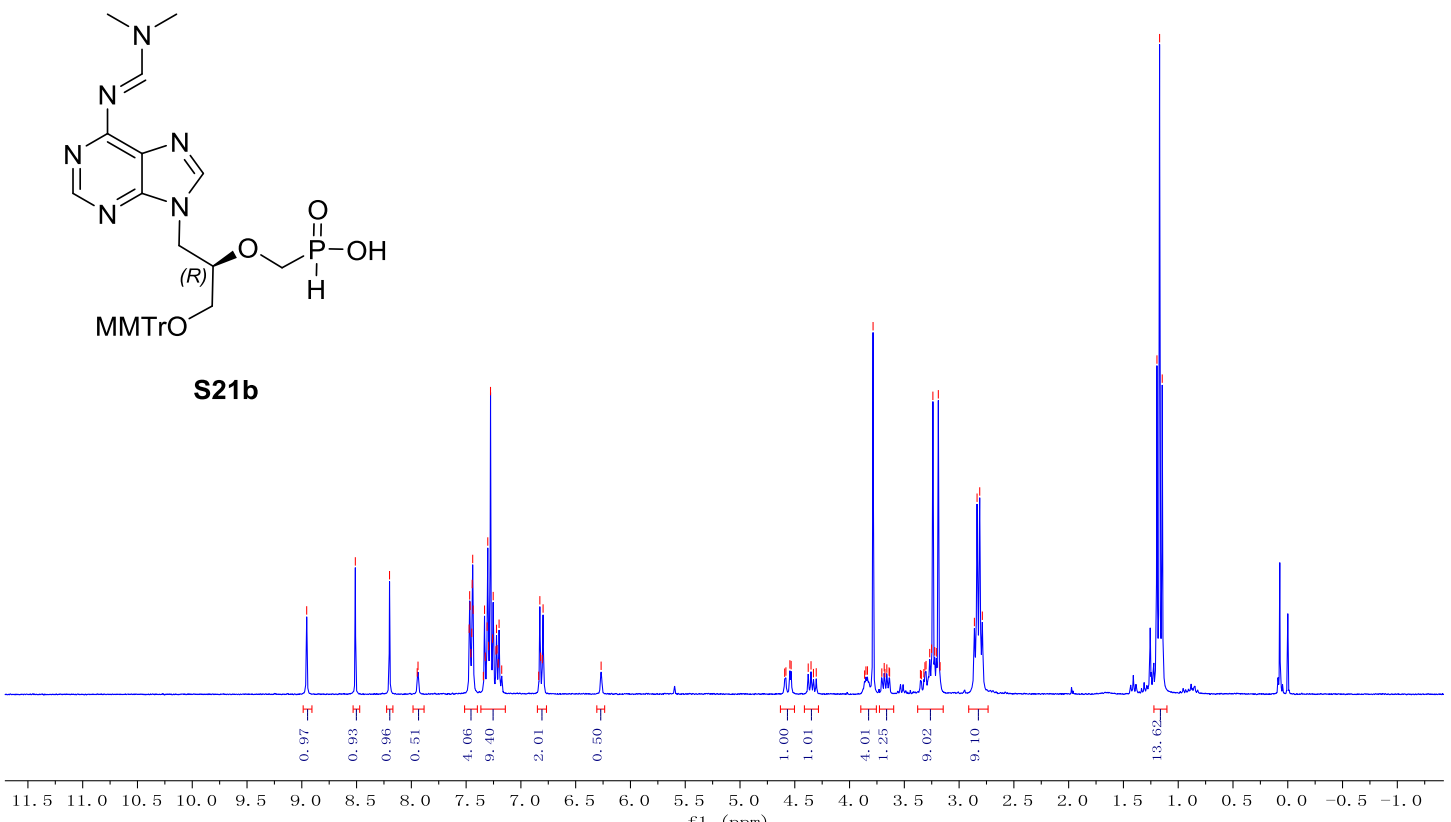



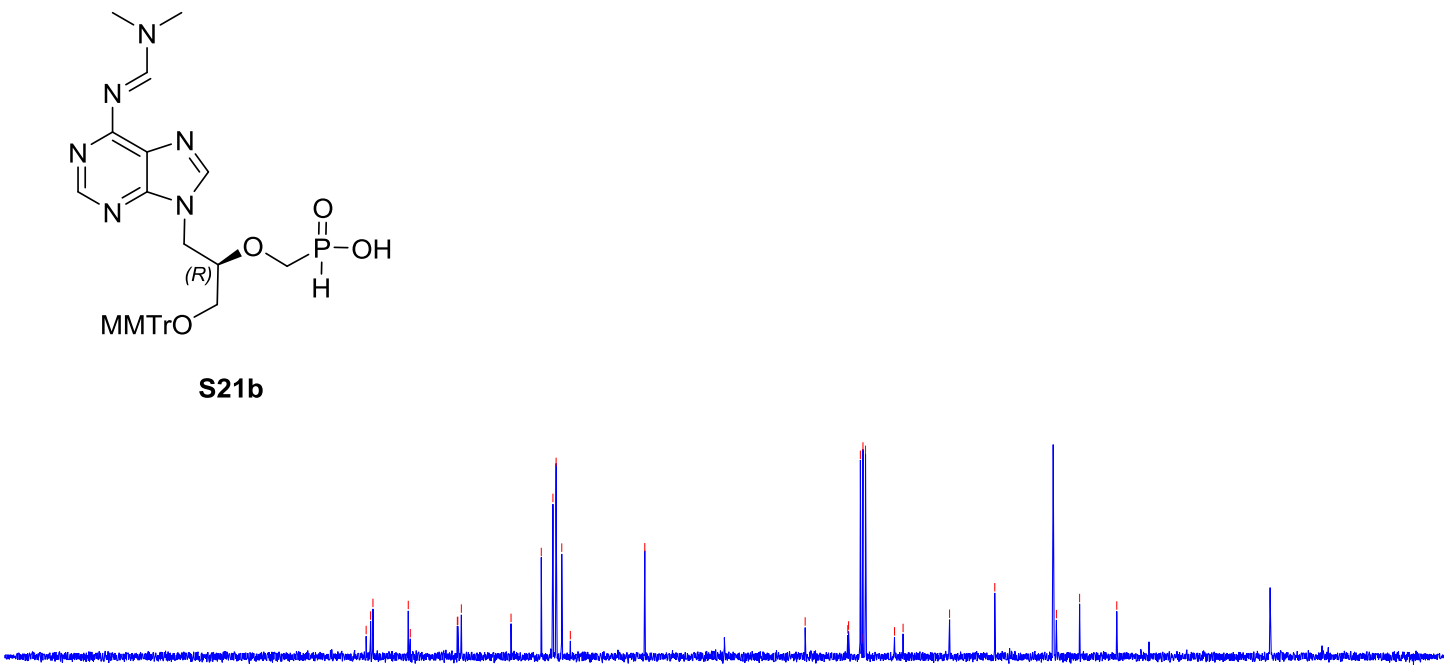

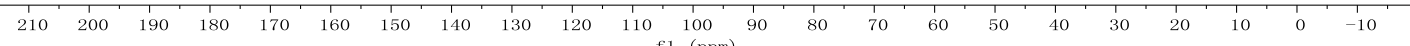

Solvent $\mathrm{CDCl} 3$

Nucleus 31P

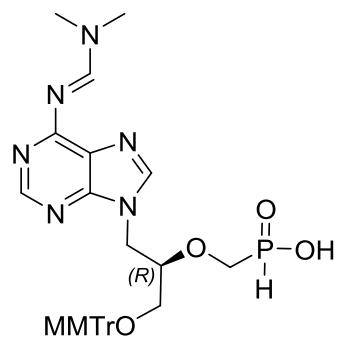

S21b

oo $\quad 180$ 


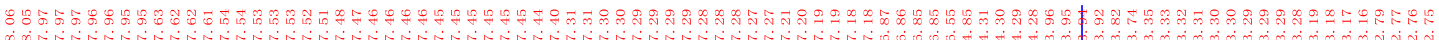
Nucleus $1 \mathrm{H}$<smiles>COC[C@H](COP(=O)(O)O)OCn1ccc(NC(=O)c2ccccc2)nc1=O</smiles>

S2a

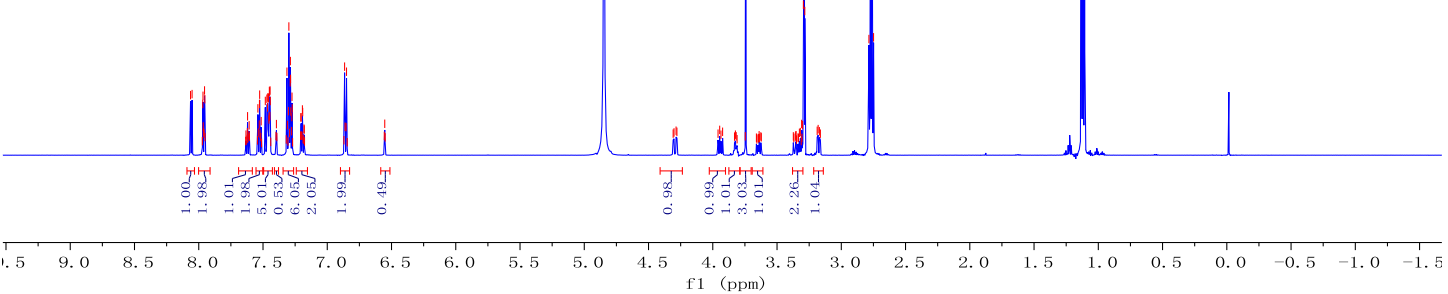

Solvent CD3OD

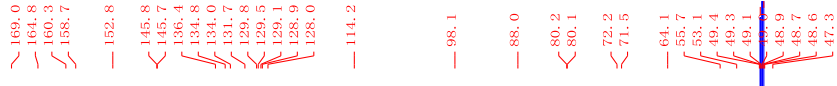

Nucleus 13C<smiles>O=C(COCCOP(=O)(O)O)c1ccccc1</smiles>

MMTrO

S2a

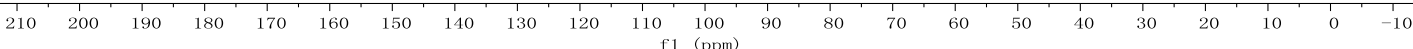



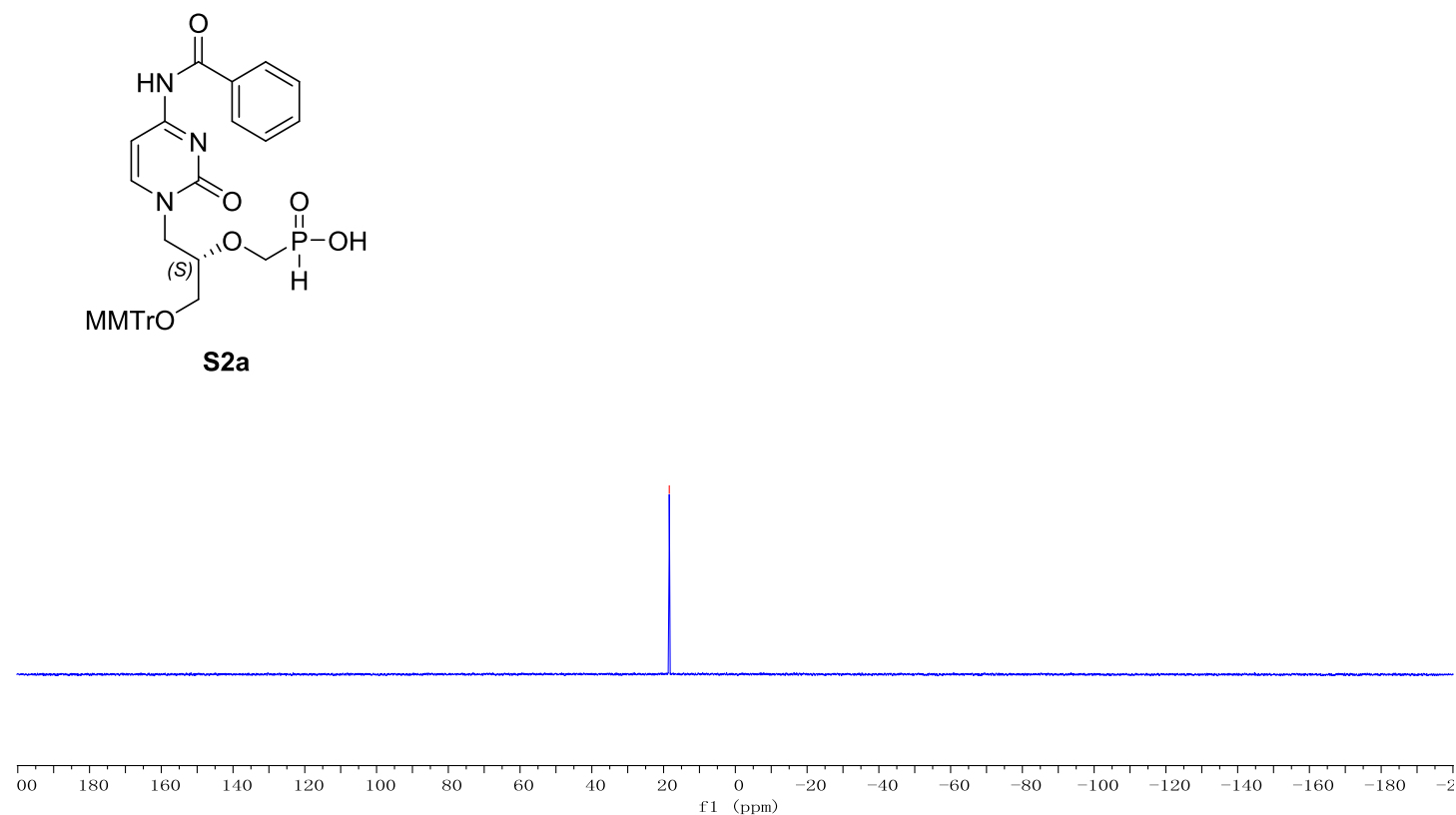

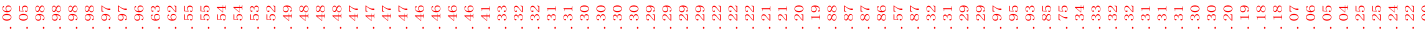
Solvent CD3OD

Nucleus $1 \mathrm{H}$<smiles>COC[C@H](Cn1ccc(NC(=O)c2ccccc2)nc1=O)OCP(=O)(O)O</smiles>

S2b

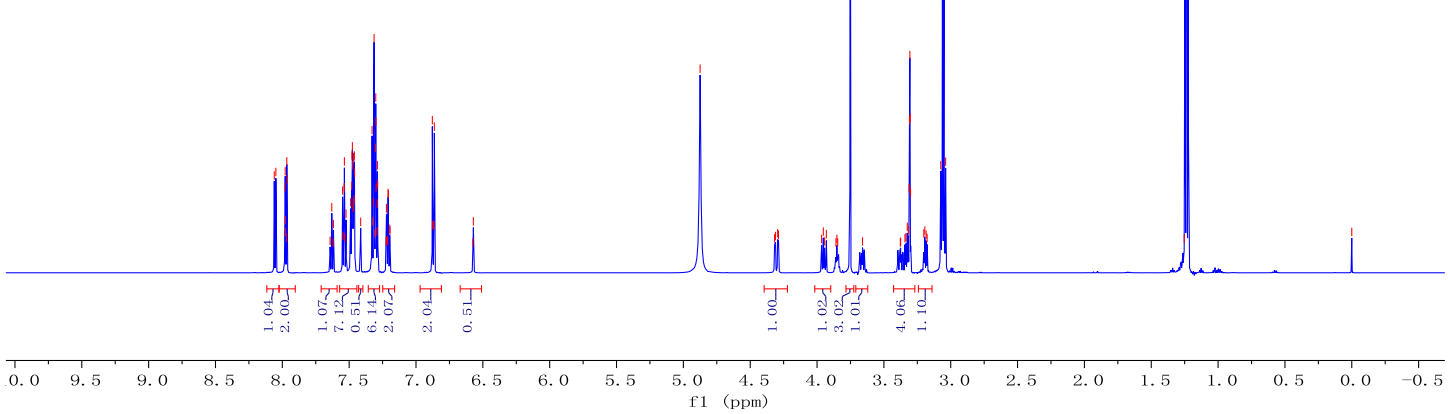




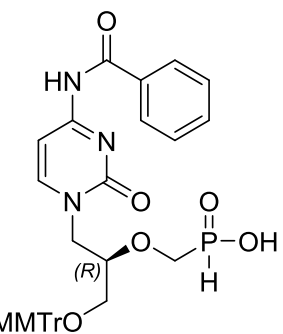

S2b

Solvent CD3OD

Nucleus 31P

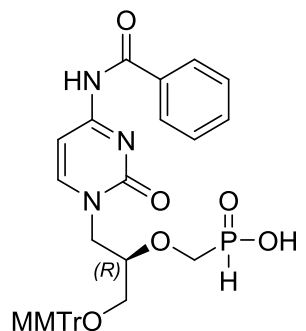

s2b

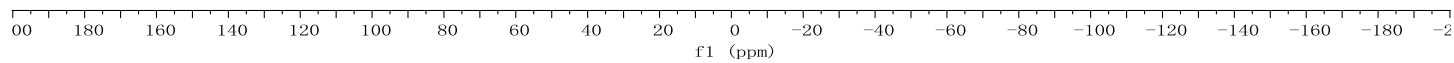




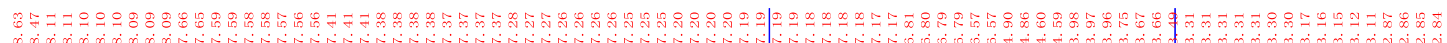
Solvent CD3OD

Nucleus 1H

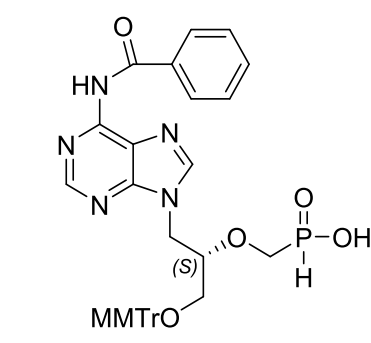

S3a
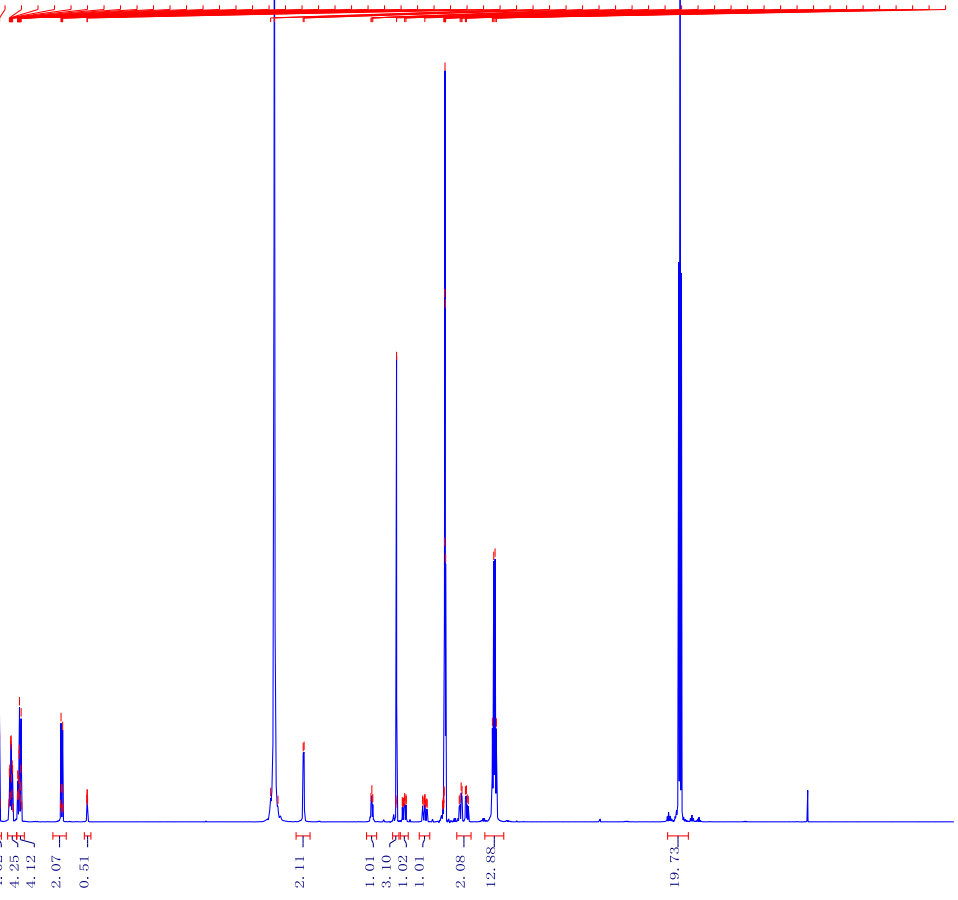

\begin{tabular}{lllllllllllllllllllllllllllllllllllllllllll}
\hline 11.5 & 11.0 & 10.5 & 10.0 & 9.5 & 9.0 & 8.5 & 8.0 & 7.5 & 7.0 & 6.5 & 6.0 & 5.5 & 5.0 & 4.5 & 4.0 & 3.5 & 3.0 & 2.5 & 2.0 & 1.5 & 1.0 & 0.5 & 0.0 & -0.5 & -1.0
\end{tabular}

Solvent CD3OD

Nucleus 13C

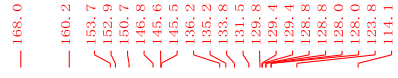<smiles>C[R16]OC[C@H](Cn1cnc2c(NC(=O)c3ccccc3)ncnc21)OCP(=O)(O)O</smiles>

S3a

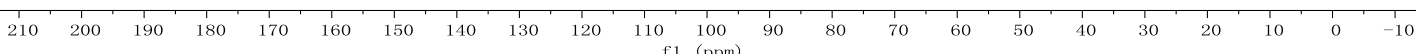




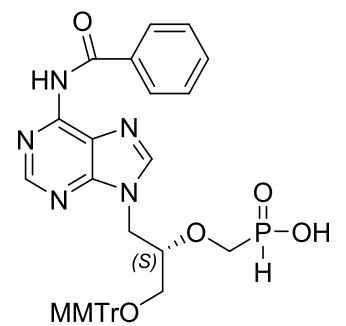

S3a

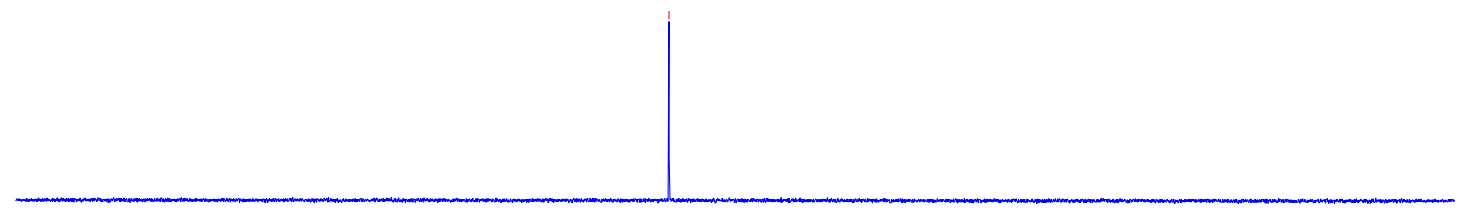

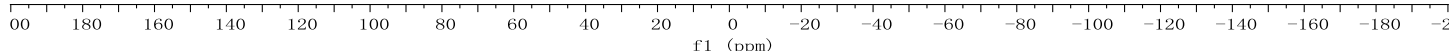

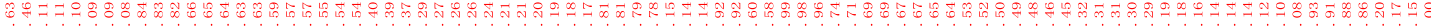
Solvent CD3OD

Nucleus $1 \mathrm{H}$

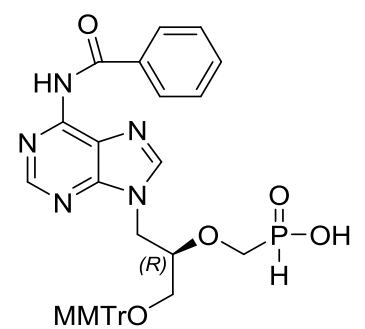

S3b

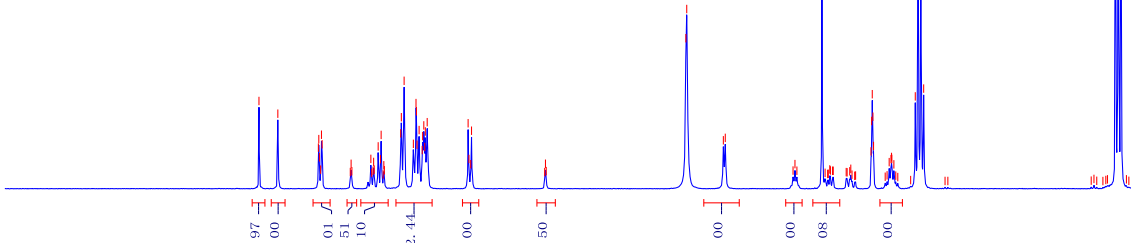

$\begin{array}{llllllllllllllllllllllllllllllllllllllll}10.5 & 10.0 & 9.5 & 9.0 & 8.5 & 8.0 & 7.5 & 7.0 & 6.5 & 6.0 & 5.5 & 5.0 & 4.5 & 4.0 & 3.5 & 3.0 & 2.5 & 2.0 & 1.5 & 1.0 & 0.5 & 0.0 & -0.5 & -1.0 & -1 .\end{array}$ 


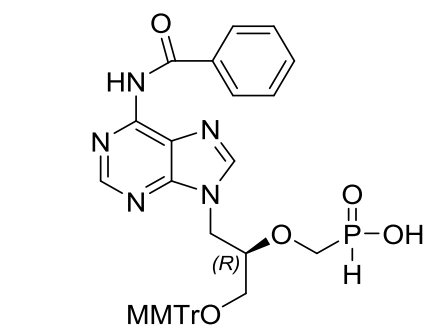

S3b

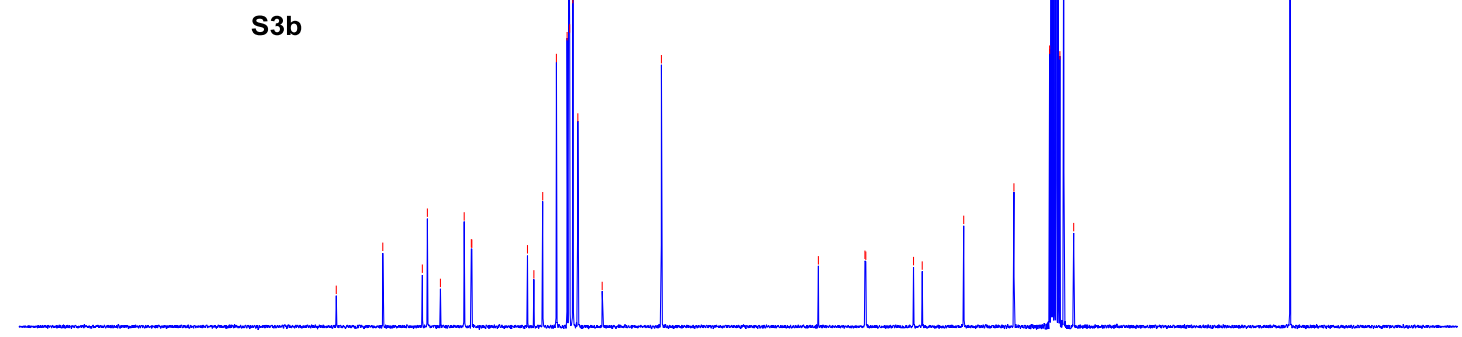

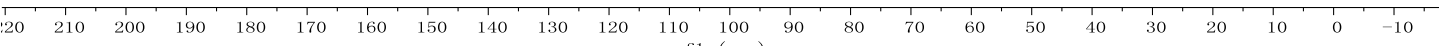

Solvent CD3OD

Nucleus 31P

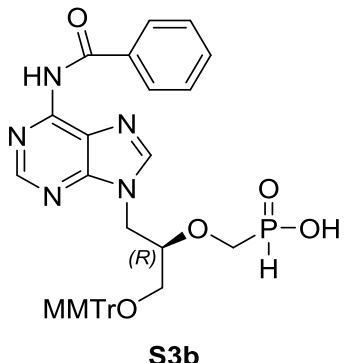

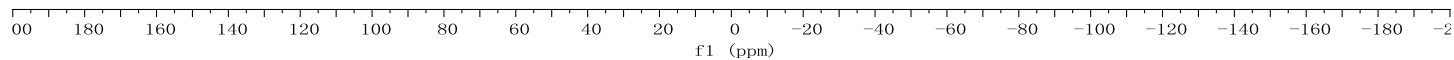




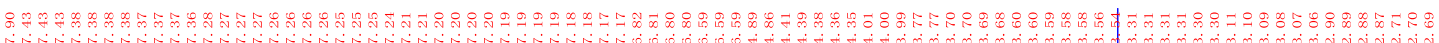
Solvent CD3OD

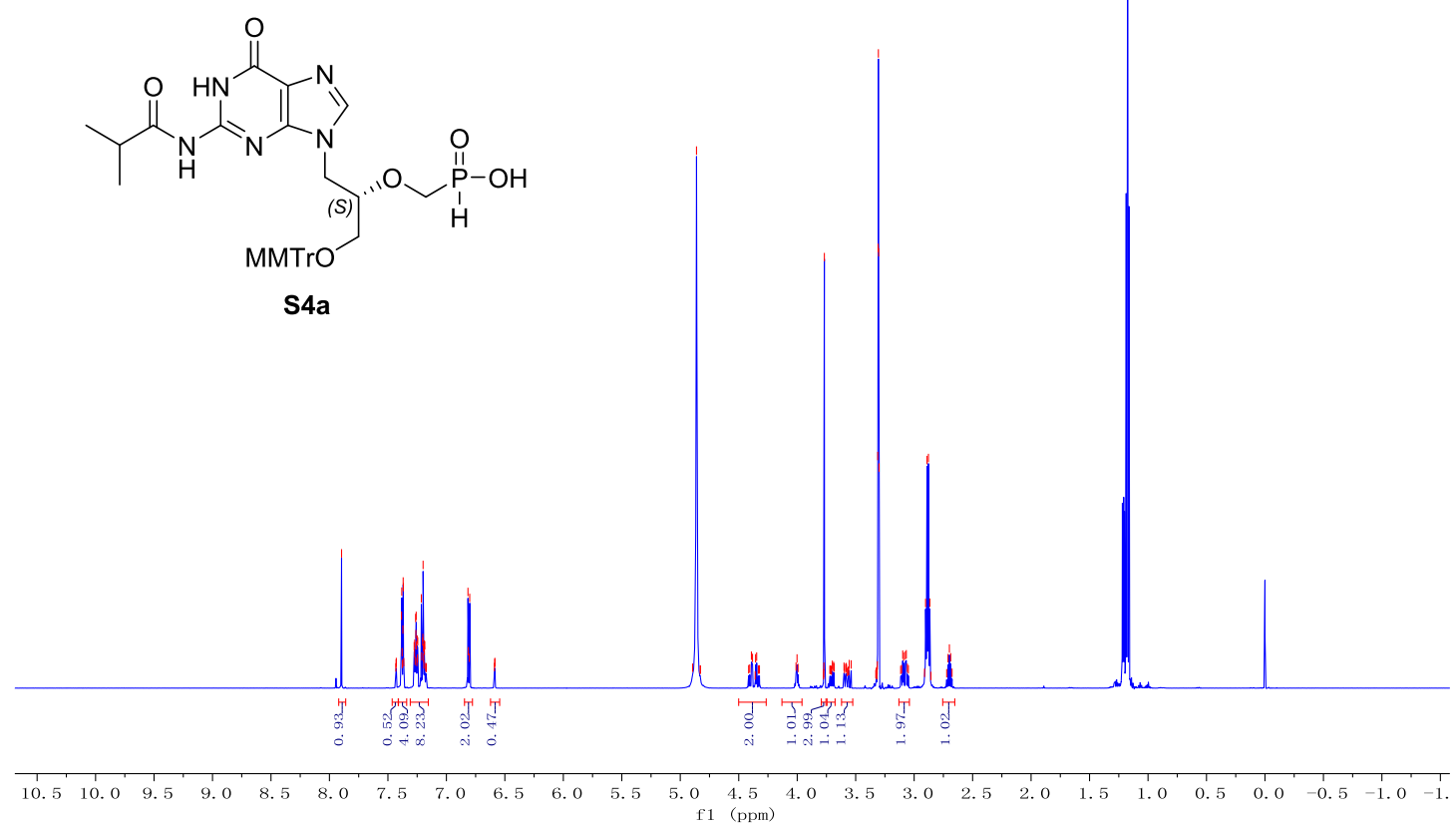
$\begin{array}{lllllll}\begin{array}{l}\text { Solvent CD3OD } \\ \text { Nucleus 13C }\end{array} & \mid\end{array}$<smiles>CC(C)C(=O)Nc1nc2c(ncn2C[C@H](O)COP(=O)(O)O)c(=O)[nH]1</smiles>

MMTrO

S4a

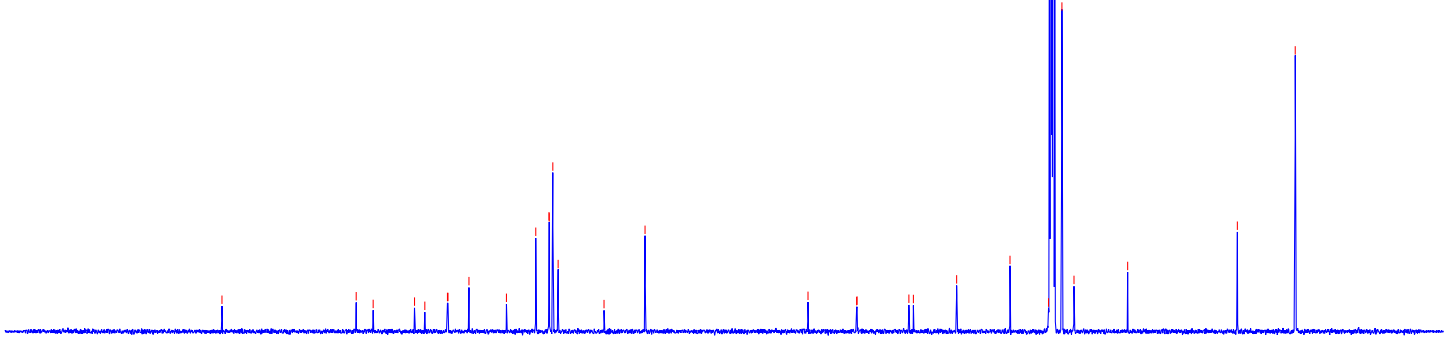

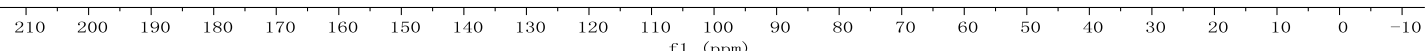




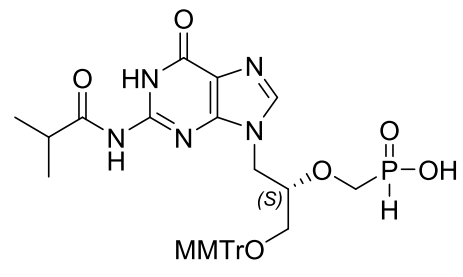

S4a

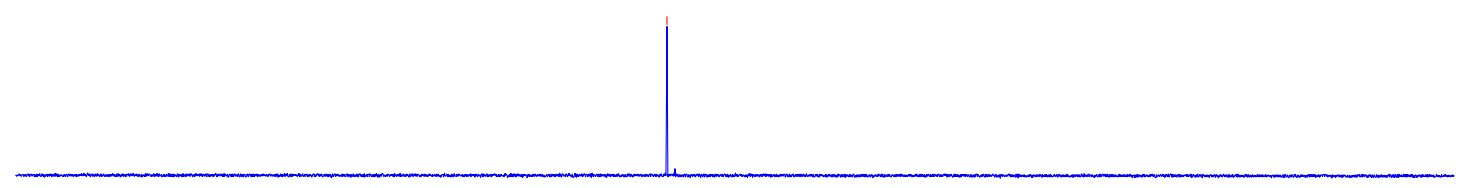

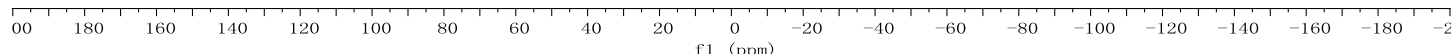

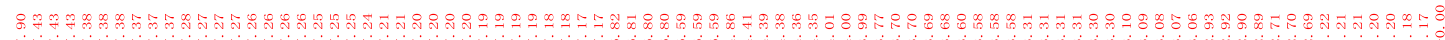
Nucleus 1H<smiles>COCC(Cn1cnc2c(=O)[nH]c(NC(=O)C(C)C)nc21)OCP(=O)(O)O</smiles>

s4b

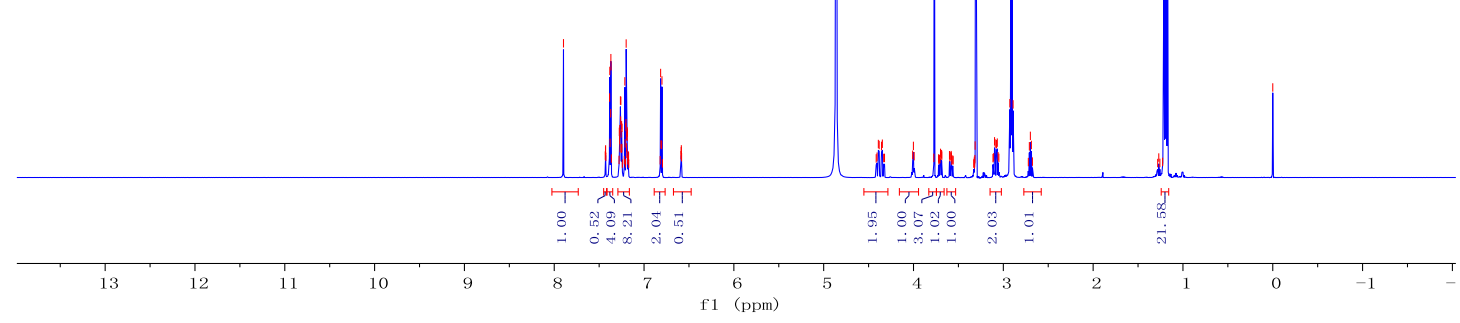



$\begin{array}{lllll}\begin{array}{l}\text { Solvent CD3OD } \\ \text { Nucleus13C }\end{array} & \mid\end{array}$<smiles>COCC(Cn1cnc2c(=O)[nH]c(NC(=O)C(C)C)nc21)OCP(=O)(O)O</smiles>

S4b

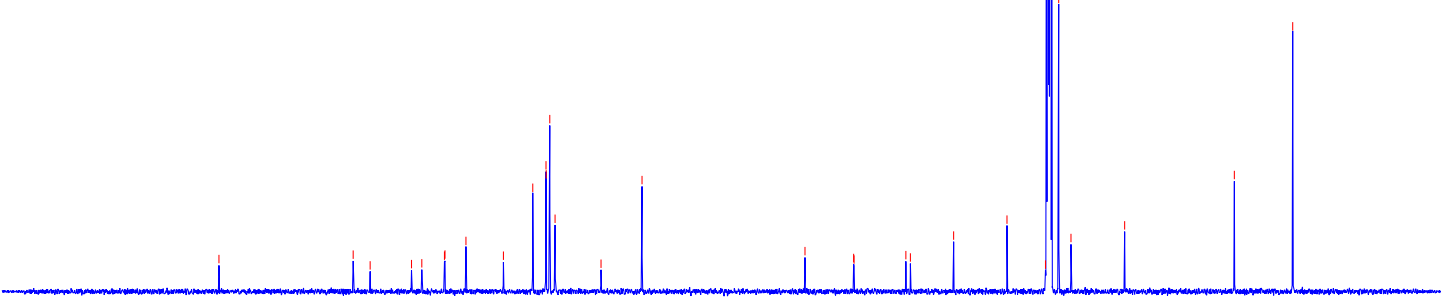

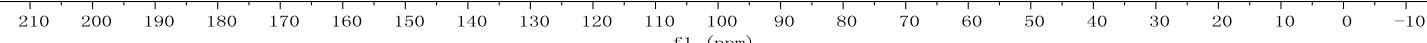

Solvent CD3OD

Nucleus 31P

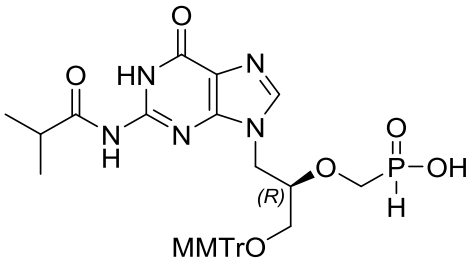

S4b

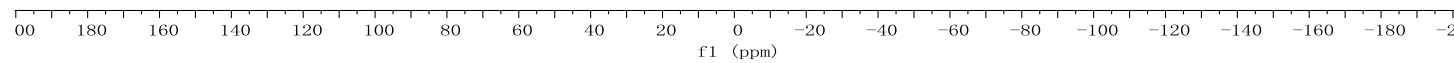




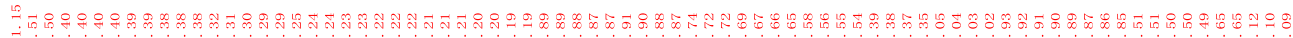
Solvent DMSO-d6 Nucleus 1H

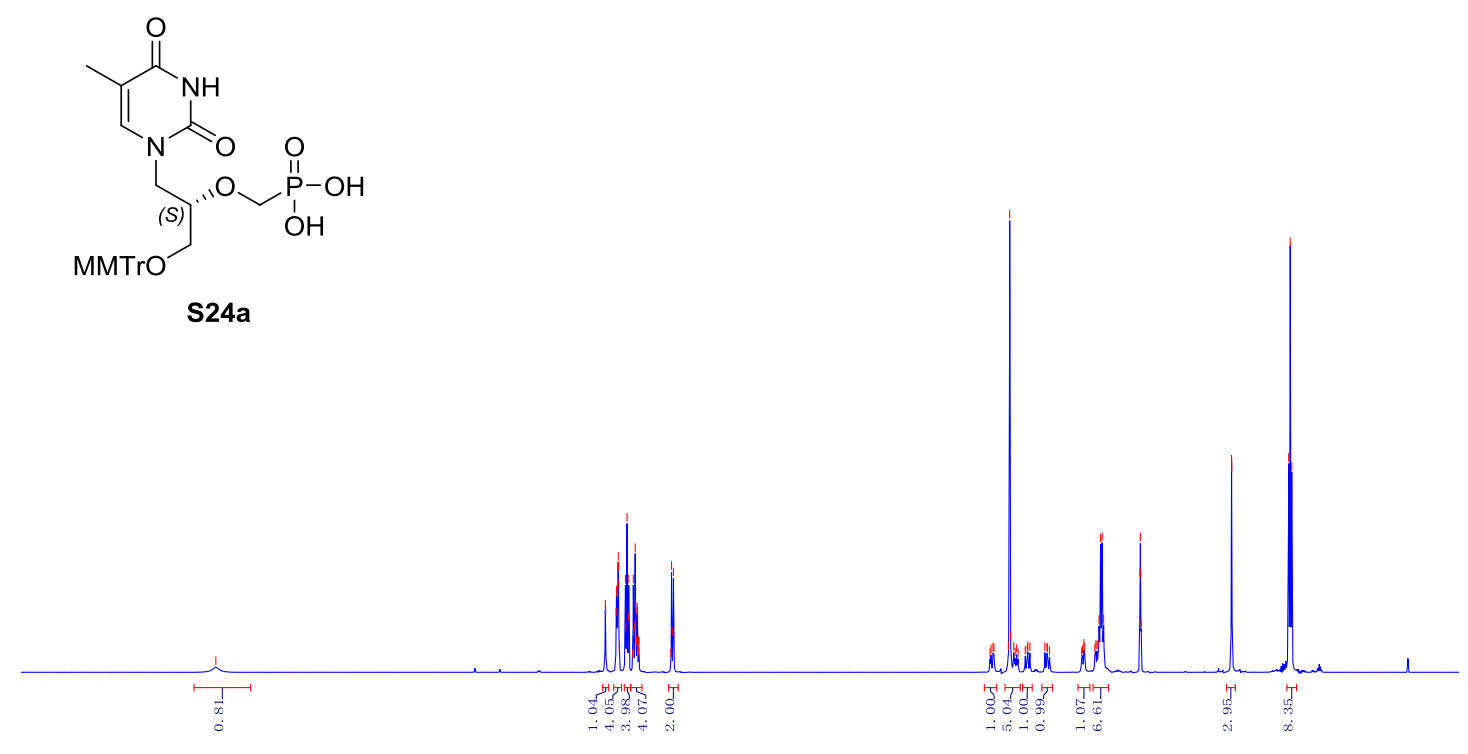

$\begin{array}{llllllllllllllllllllllllllllllllllll}12.5 & 12.0 & 11.5 & 11.0 & 10.5 & 10.0 & 9.5 & 9.0 & 8.5 & 8.0 & 7.5 & 7.0 & 6.5 & 6.0 & 5.5 & 5.0 & 4.5 & 4.0 & 3.5 & 3.0 & 2.5 & 2.0 & 1.5 & 1.0 & 0.5 & 0.0\end{array}$

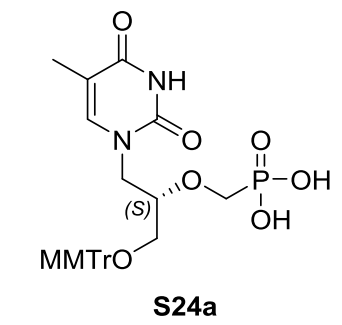



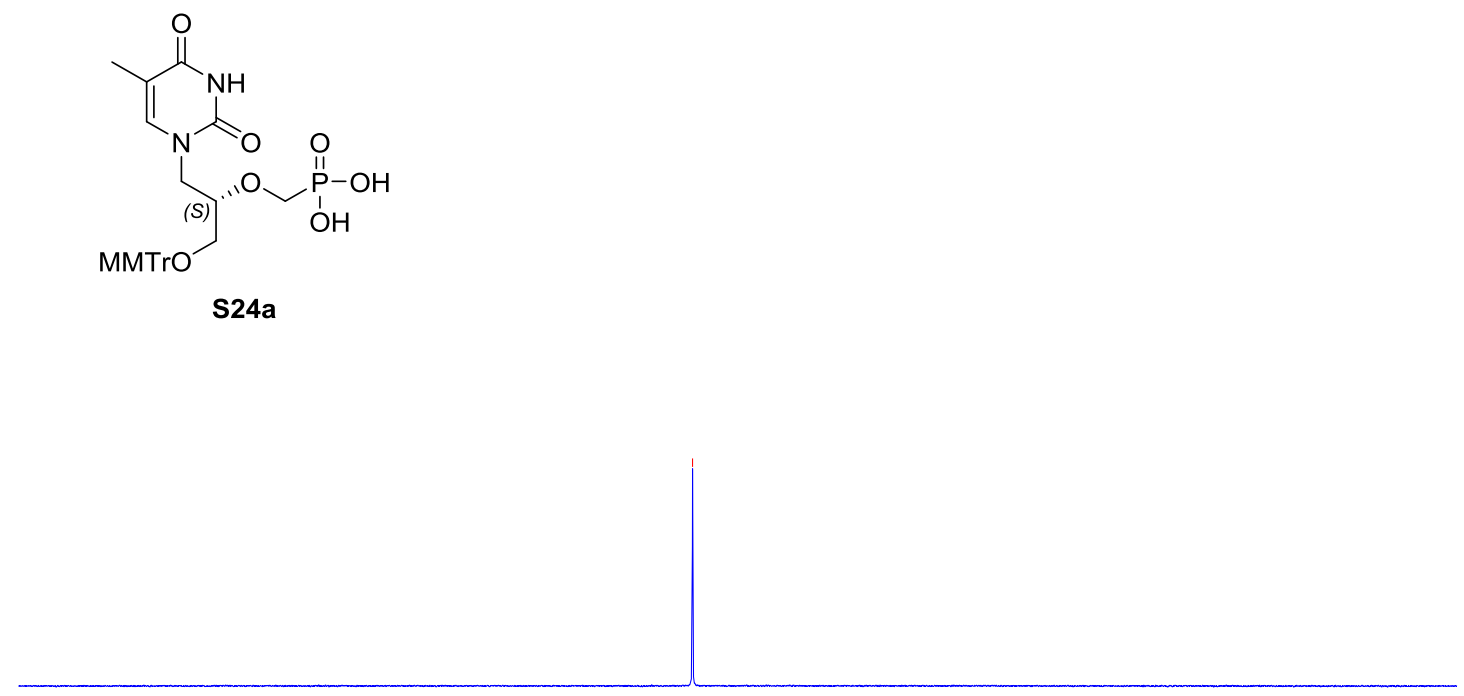

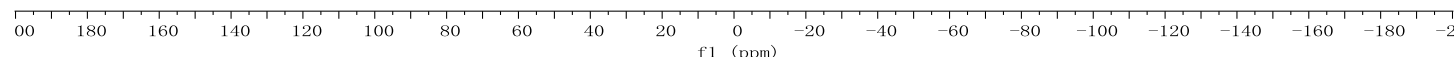

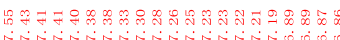

Solvent DMSO-d6

Nucleus 1H

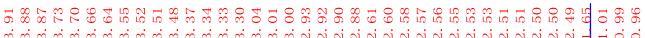

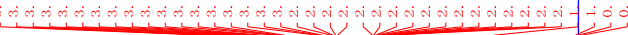

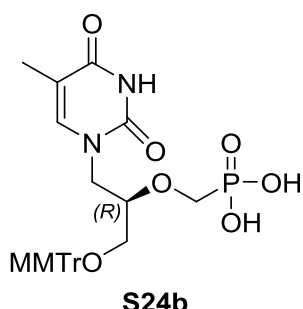

S24b

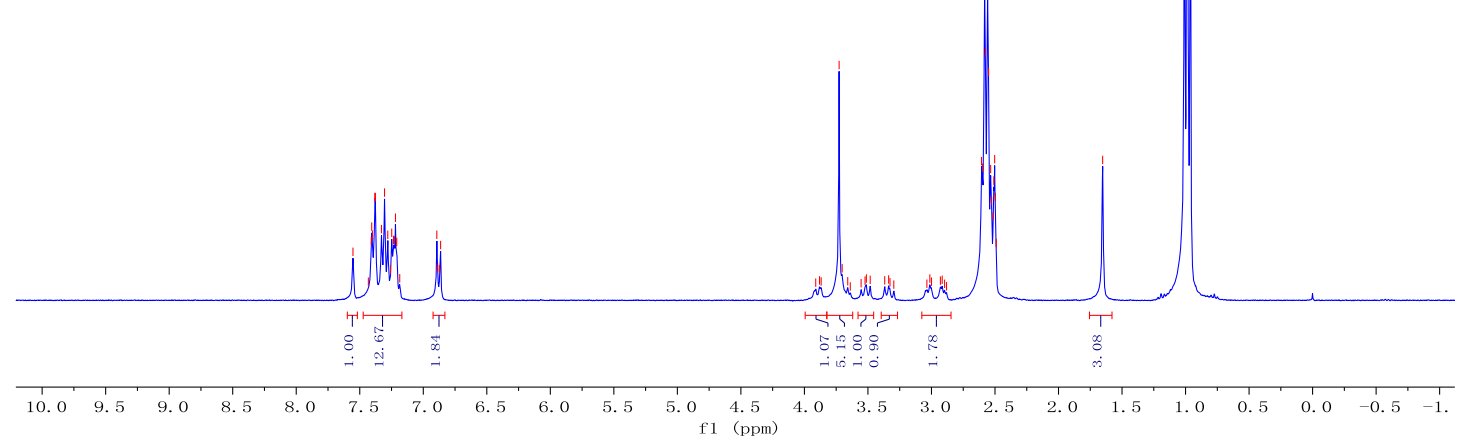



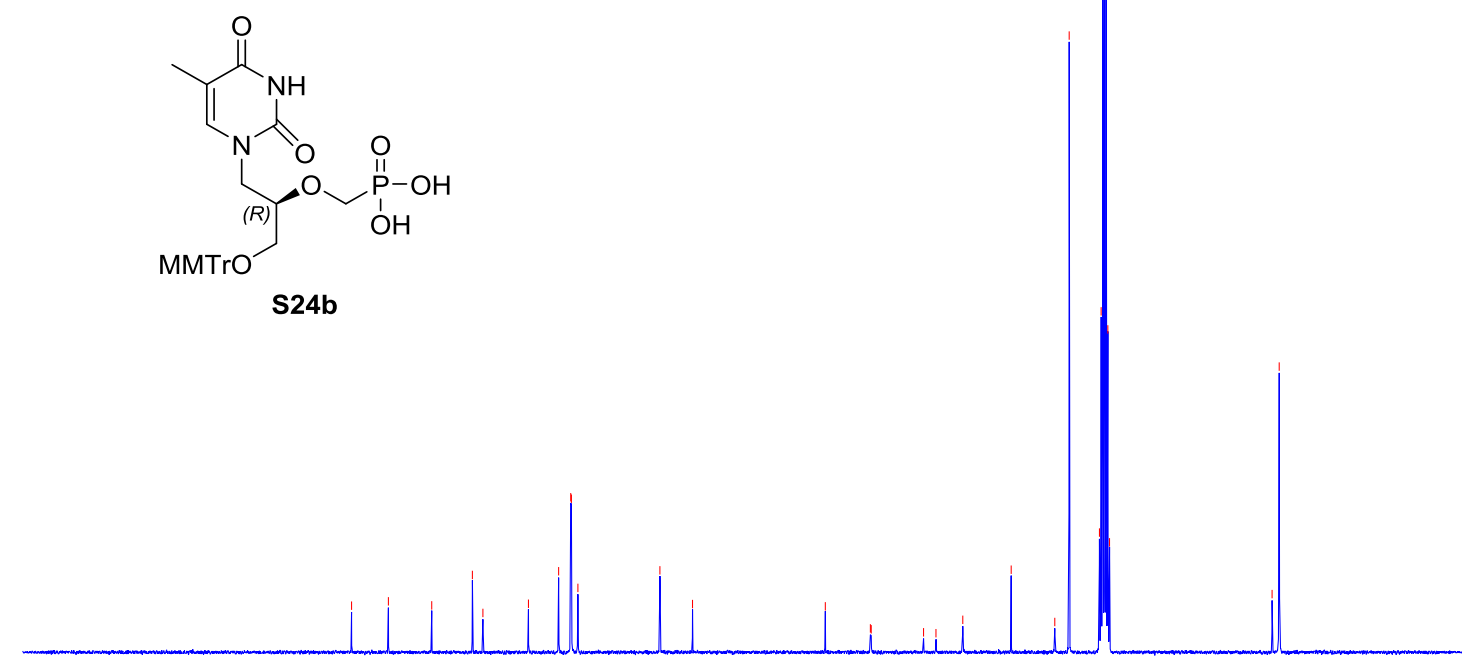

$\begin{array}{lllllllllllllllllllllllllllllllll}1 & 10 & 200 & 190 & 180 & 170 & 160 & 150 & 140 & 130 & 120 & 110 & 100 & 90 & 80 & 70 & 60 & 50 & 40 & 30 & 20 & 10 & 0 & -10\end{array}$

Solvent DMSO-d6

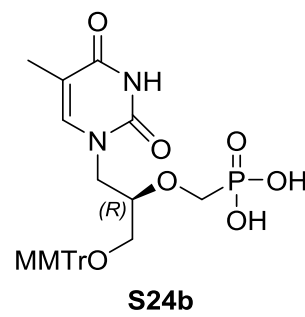

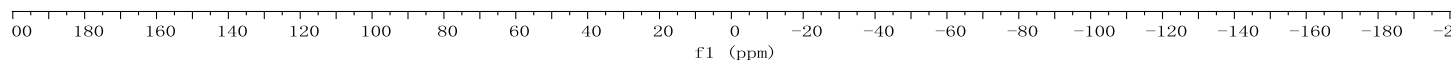



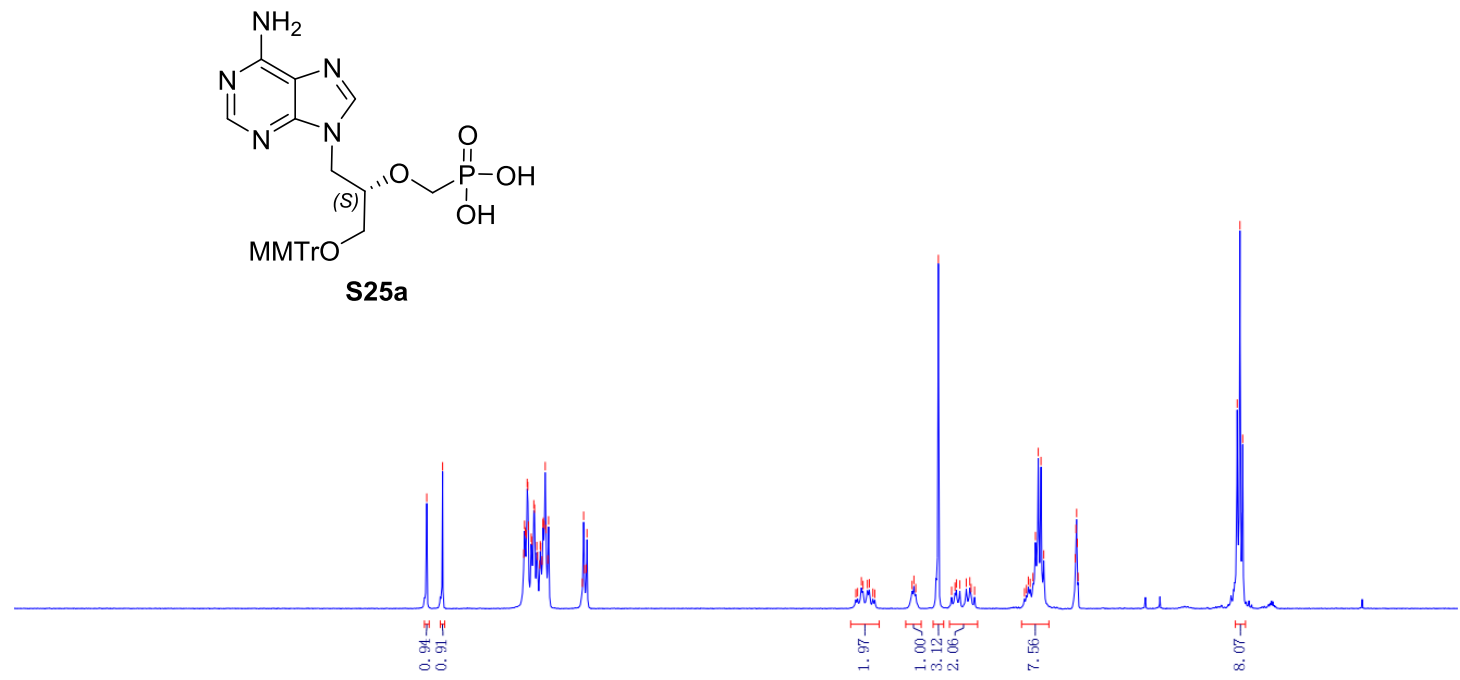

$\begin{array}{lllllllllllllllllllllllll}11.5 & 11.0 & 10.5 & 10.0 & 9.5 & 9.0 & 8.5 & 8.0 & 7.5 & 7.0 & 6.5 & 6.0 & 5.5 & 5.0 & 4.5 & 4.0 & 3.5 & 3.0 & 2.5 & 2.0 & 1.5 & 1.0 & 0.5 & 0.0 & -0.5\end{array}$

Solvent DMSO- $d 6$

Nucleus $13 \mathrm{C}$

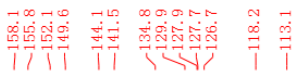

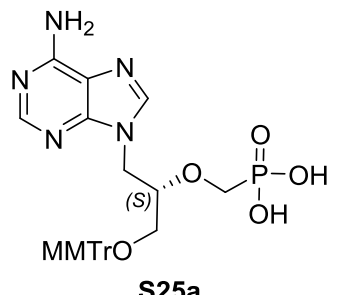

S25a

$\begin{array}{lllllllllllllllllllllll}210 & 200 & 190 & 180 & 170 & 160 & 150 & 140 & 130 & 120 & 110 & 100 & 90 & 80 & 70 & 60 & 50 & 40 & 30 & 20 & 10 & 0 & -10\end{array}$ 
Solvent DMSO- $d 6$

Nucleus 31P
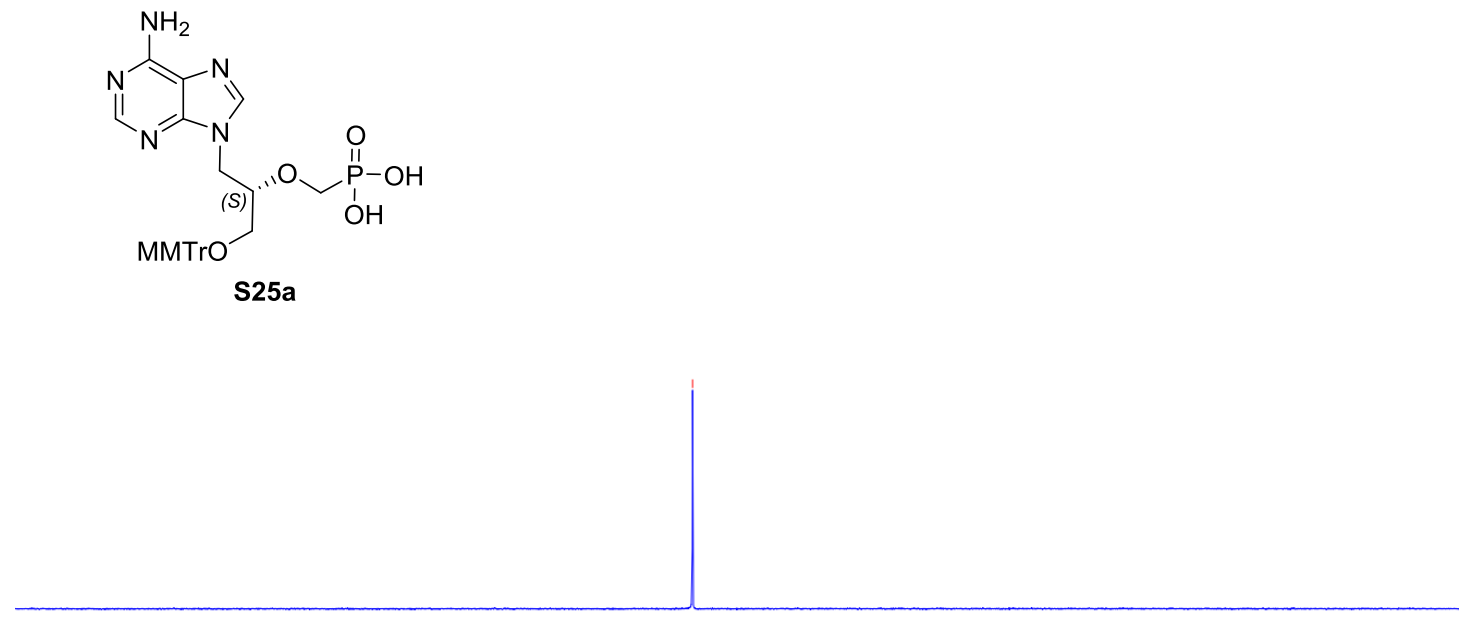

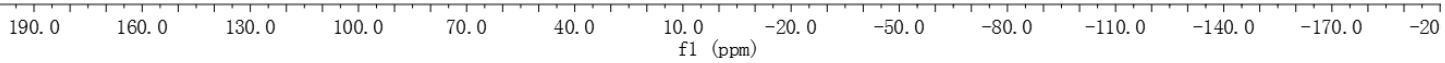

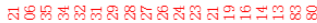

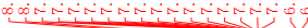

Solvent DMSO-d 6

Nucleus $1 \mathrm{H}$
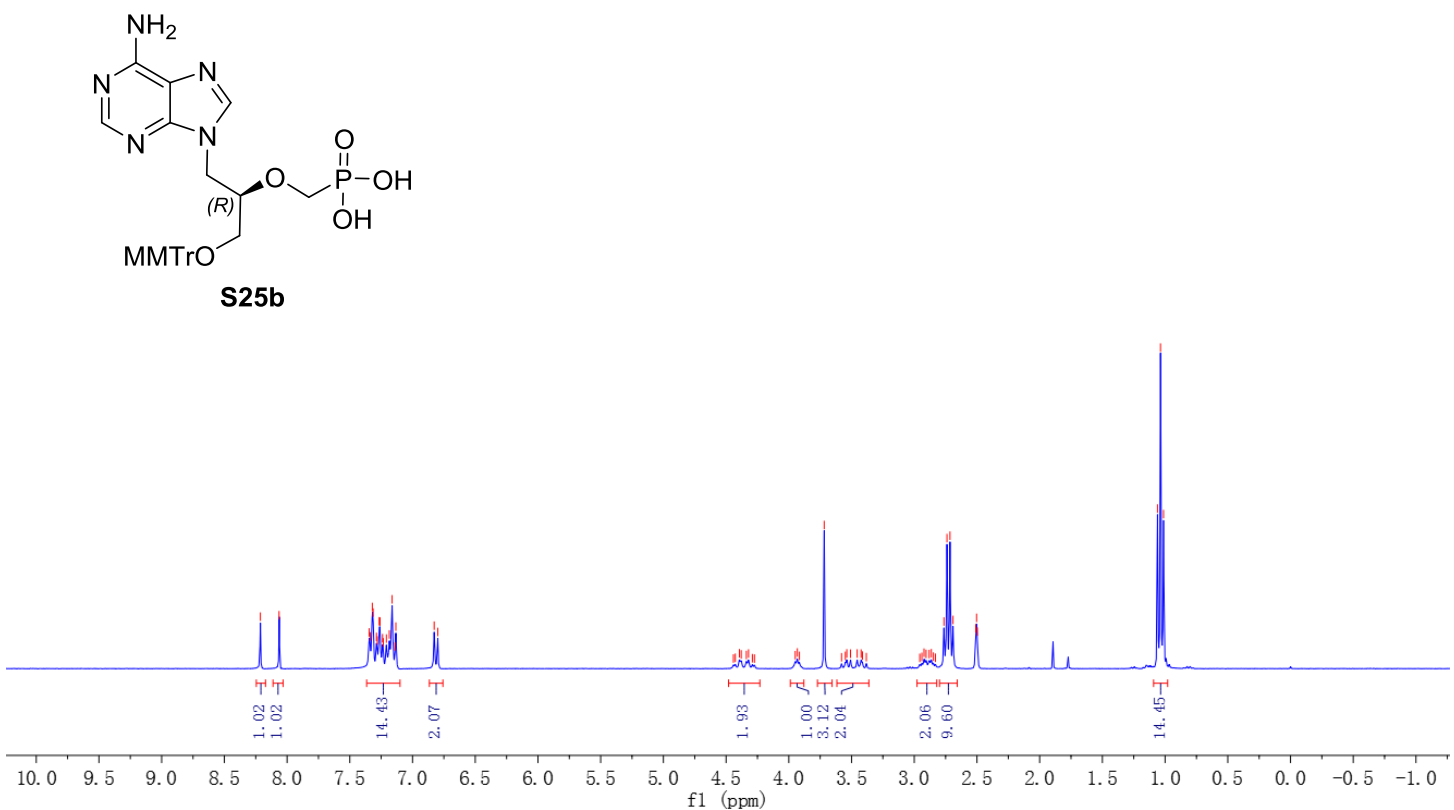
<smiles>COC[C@H](COP(=O)(O)O)OCn1cnc2c(N)ncnc21</smiles>

S25b

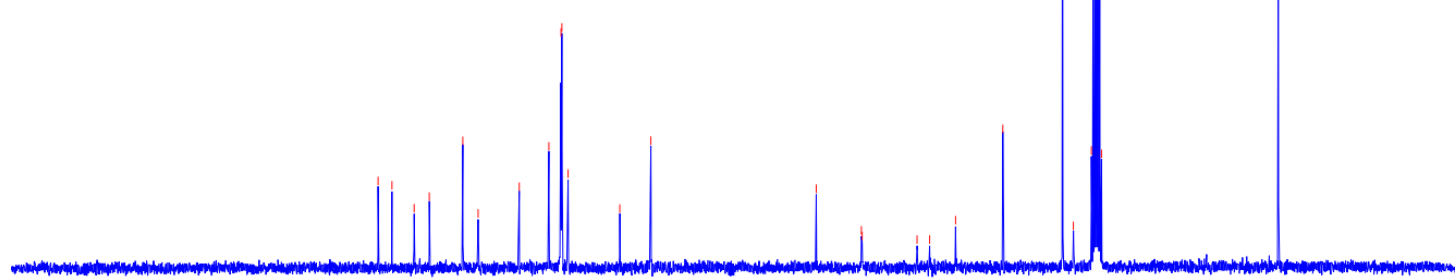

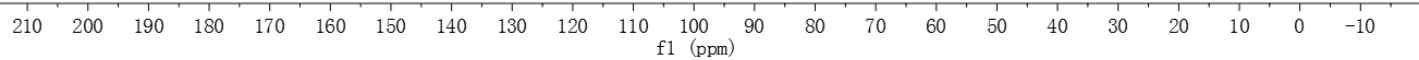

Solvent DMSO- $d 6$

Nucleus $31 \mathrm{P}$<smiles>COC[C@H](COP(=O)(O)O)OCn1cnc2c(N)ncnc21</smiles>

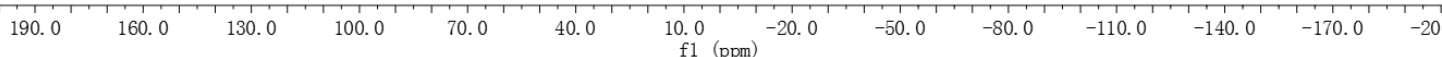




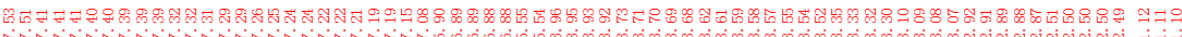
WREM Solvent DMSO-d 6

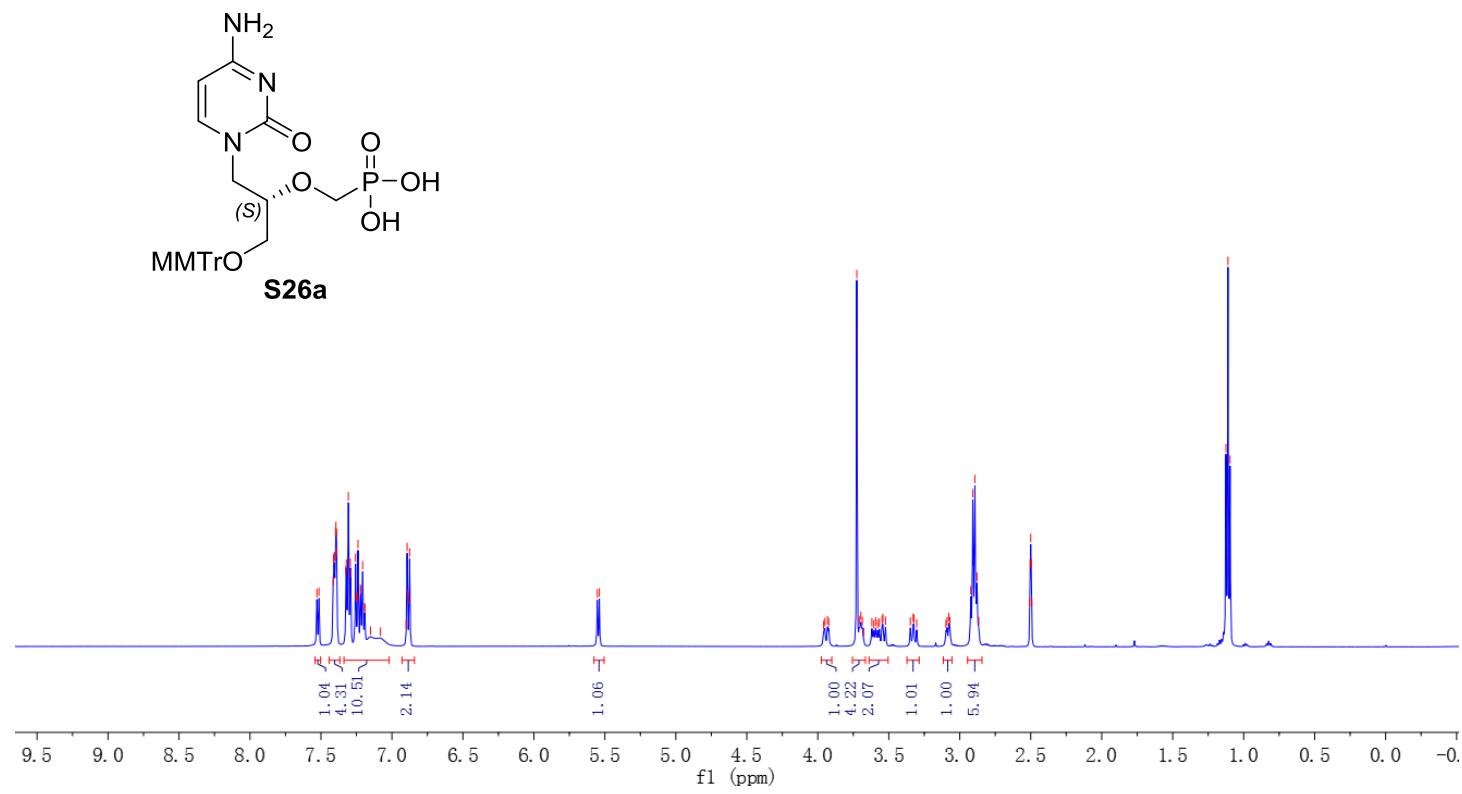

Solvent DMSO- $d 6$ Nucleus $13 \mathrm{C}$<smiles>Nc1ccn(CCOP(=O)(O)O)c(=O)n1</smiles>

MMTrO'

S26a

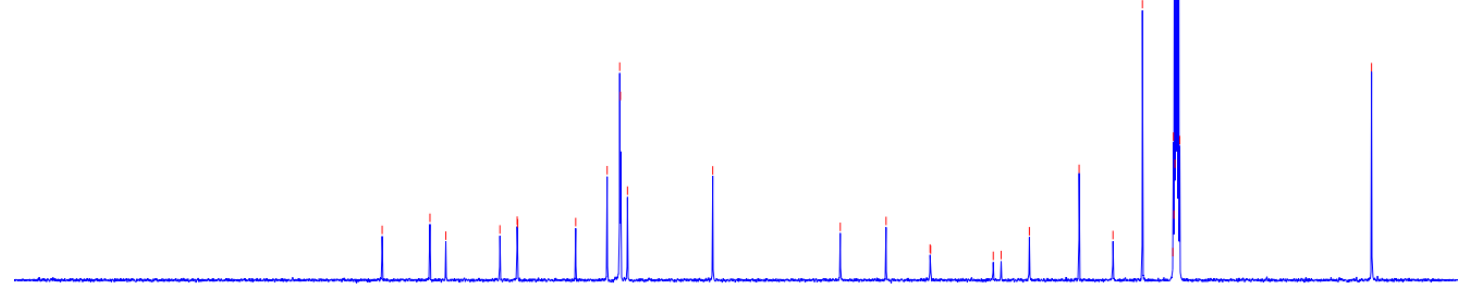

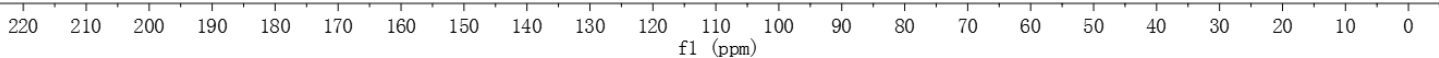


Solvent DMSO- $d 6$

Nucleus 13C<smiles>COC[C@H](COP(=O)(O)O)OCn1ccc(N)nc1=O</smiles>

S26a

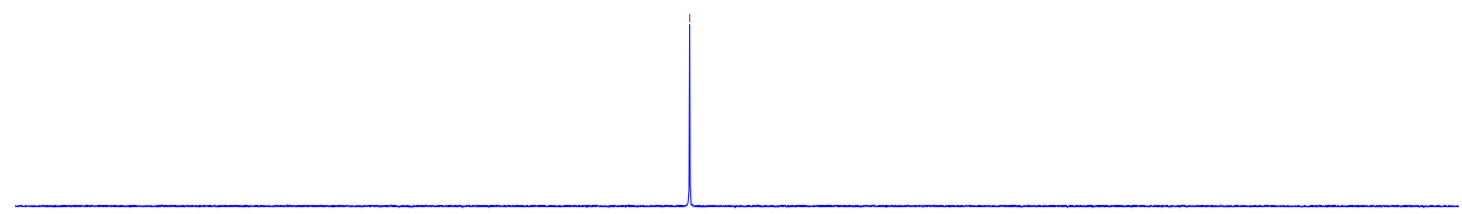

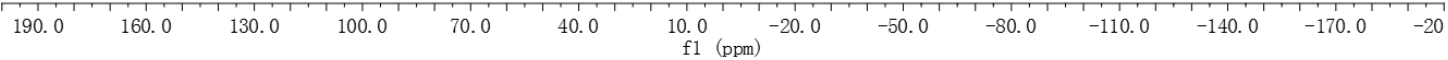

Solvent DMSO-d 6 Nucleus 1H<smiles>COC[C@@H](OCn1ccc(N)nc1=O)OCP(=O)(O)O</smiles>

s26b 
<smiles>COCCOCCn1ccc(N)nc1=O</smiles>

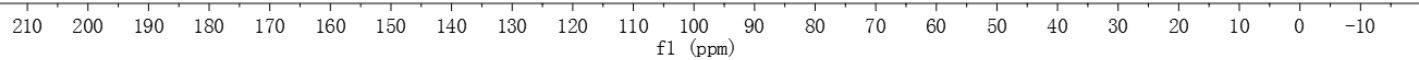

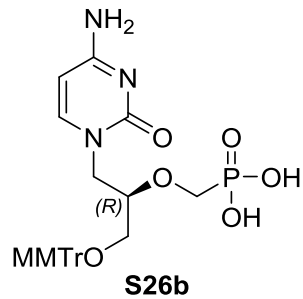

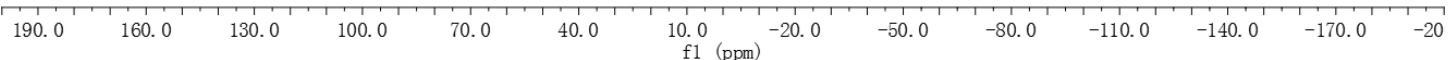




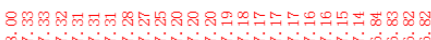
Fition Solvent DMSO- $d 6$

Nucleus $1 \mathrm{H}$
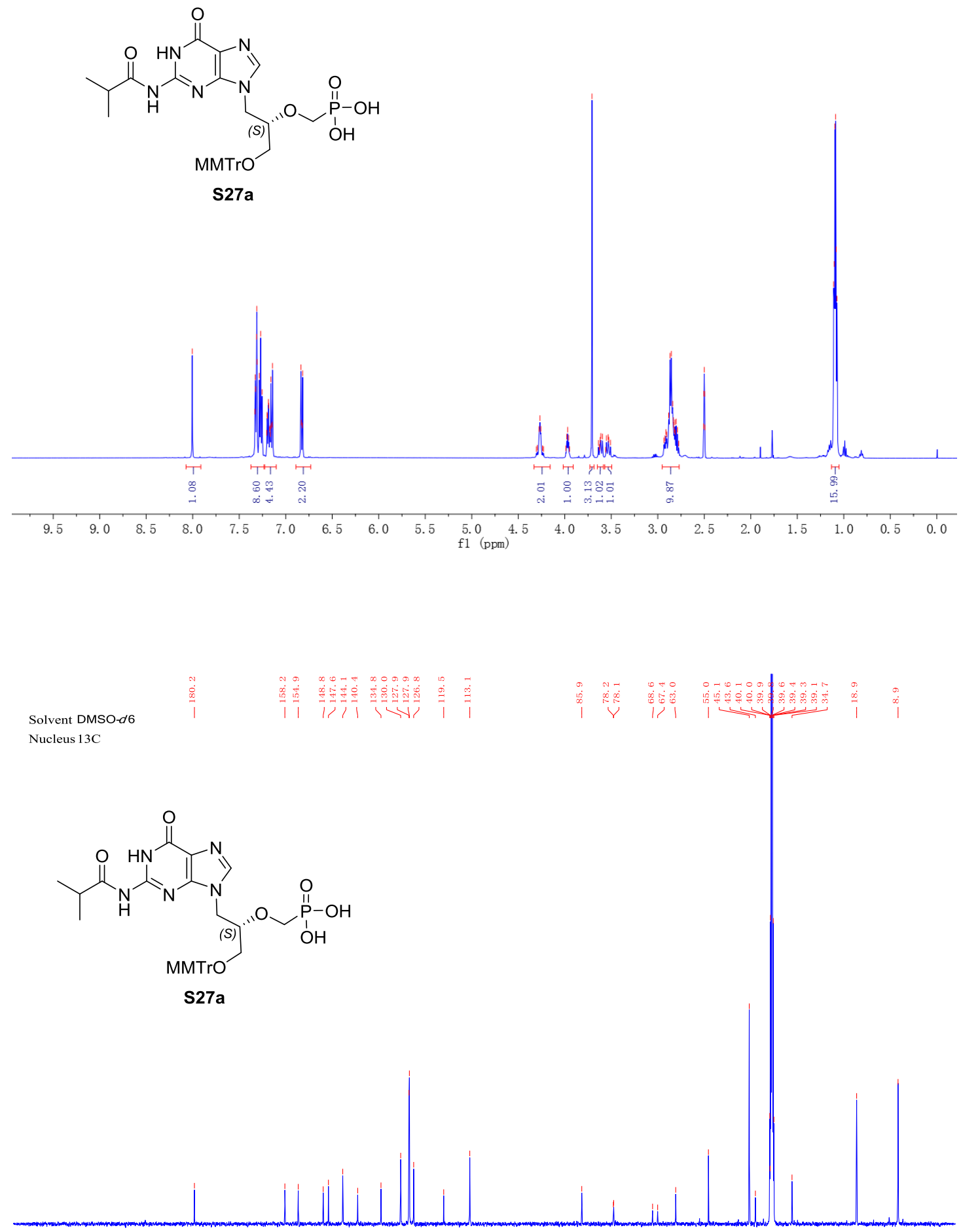

S27a

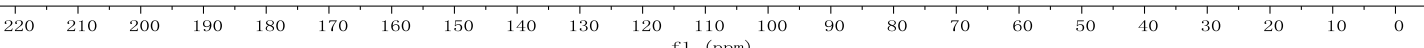



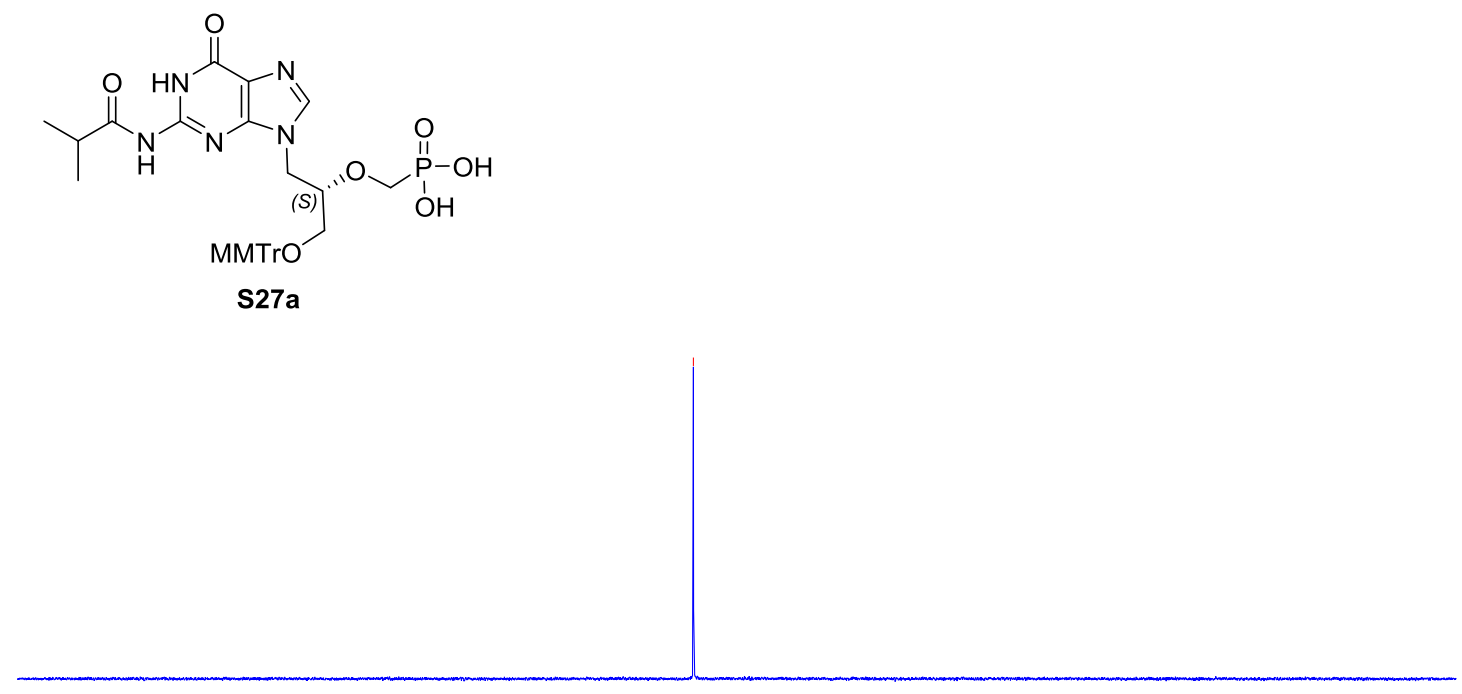

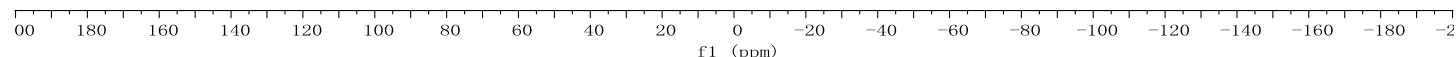

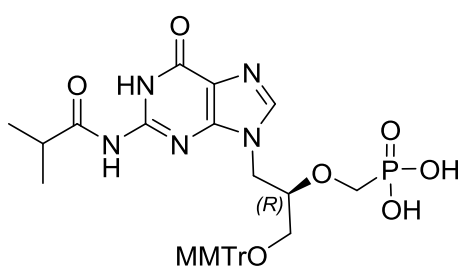

S27b

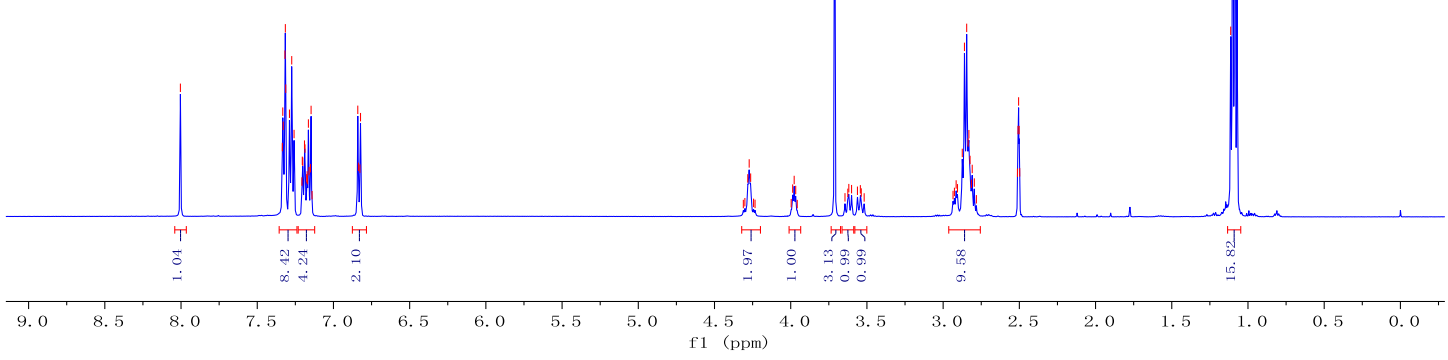



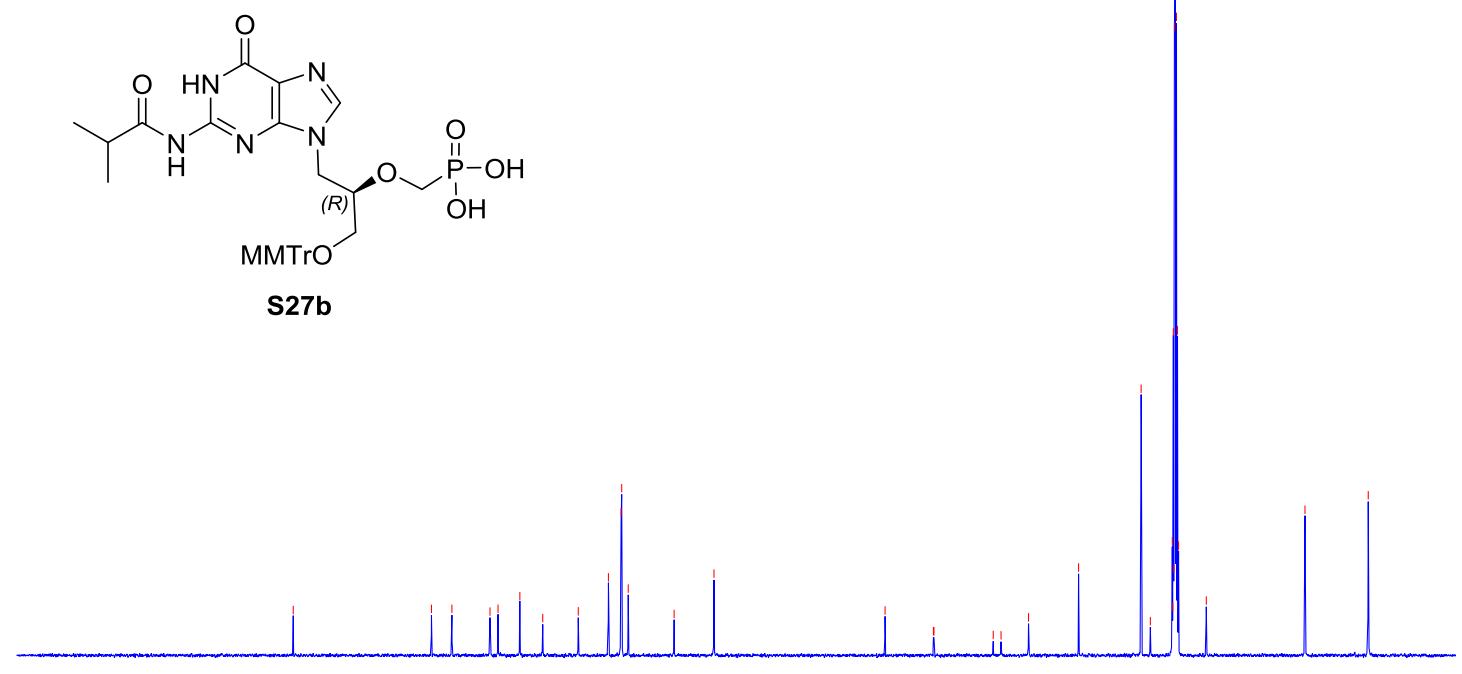

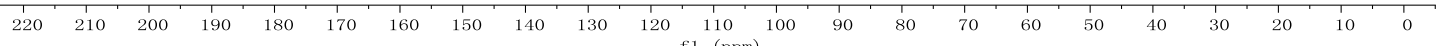

Solvent DMSO-d6

Nucleus 31P

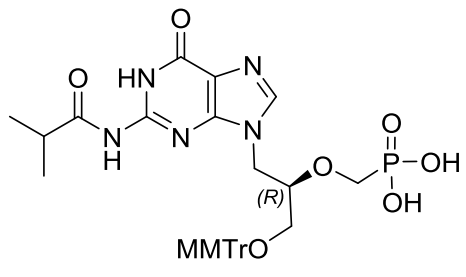

S27b

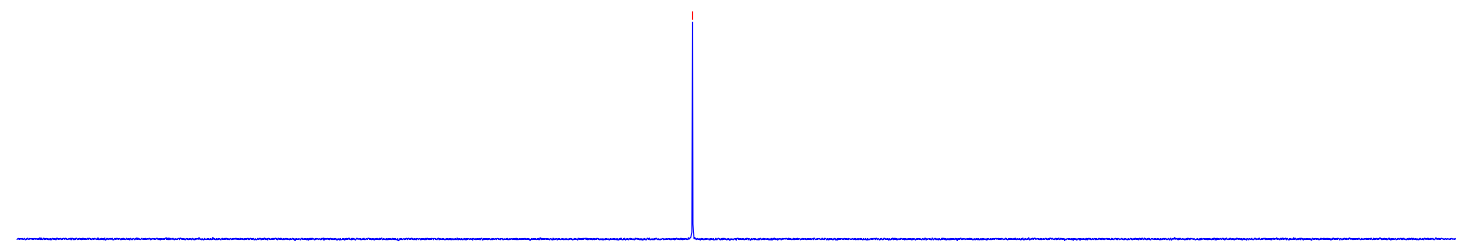

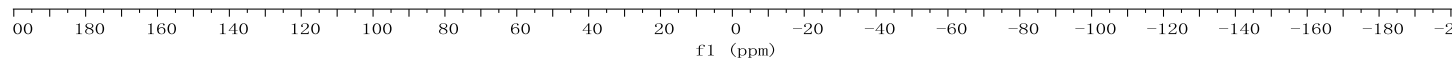


Solvent DMSO-d6 Nucleus $1 \mathrm{H}$
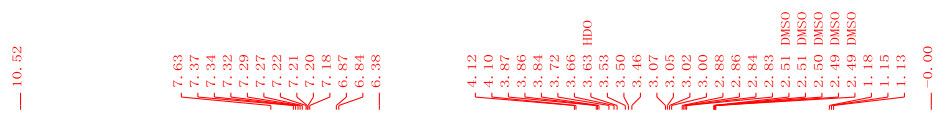
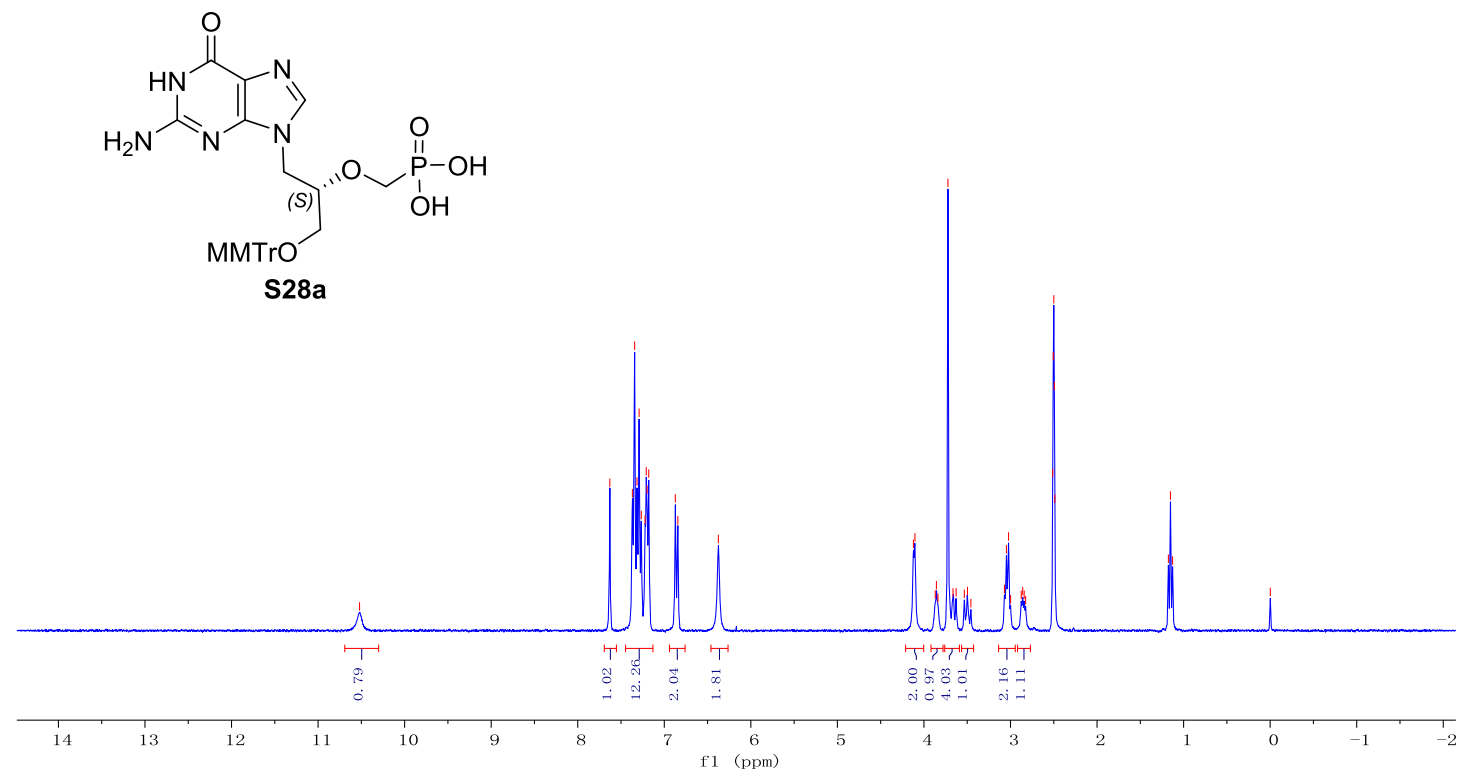

Solvent DMSO-d6

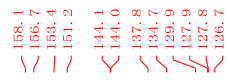

$\stackrel{\circ}{\stackrel{0}{\infty}=}$

Nucleus 13C
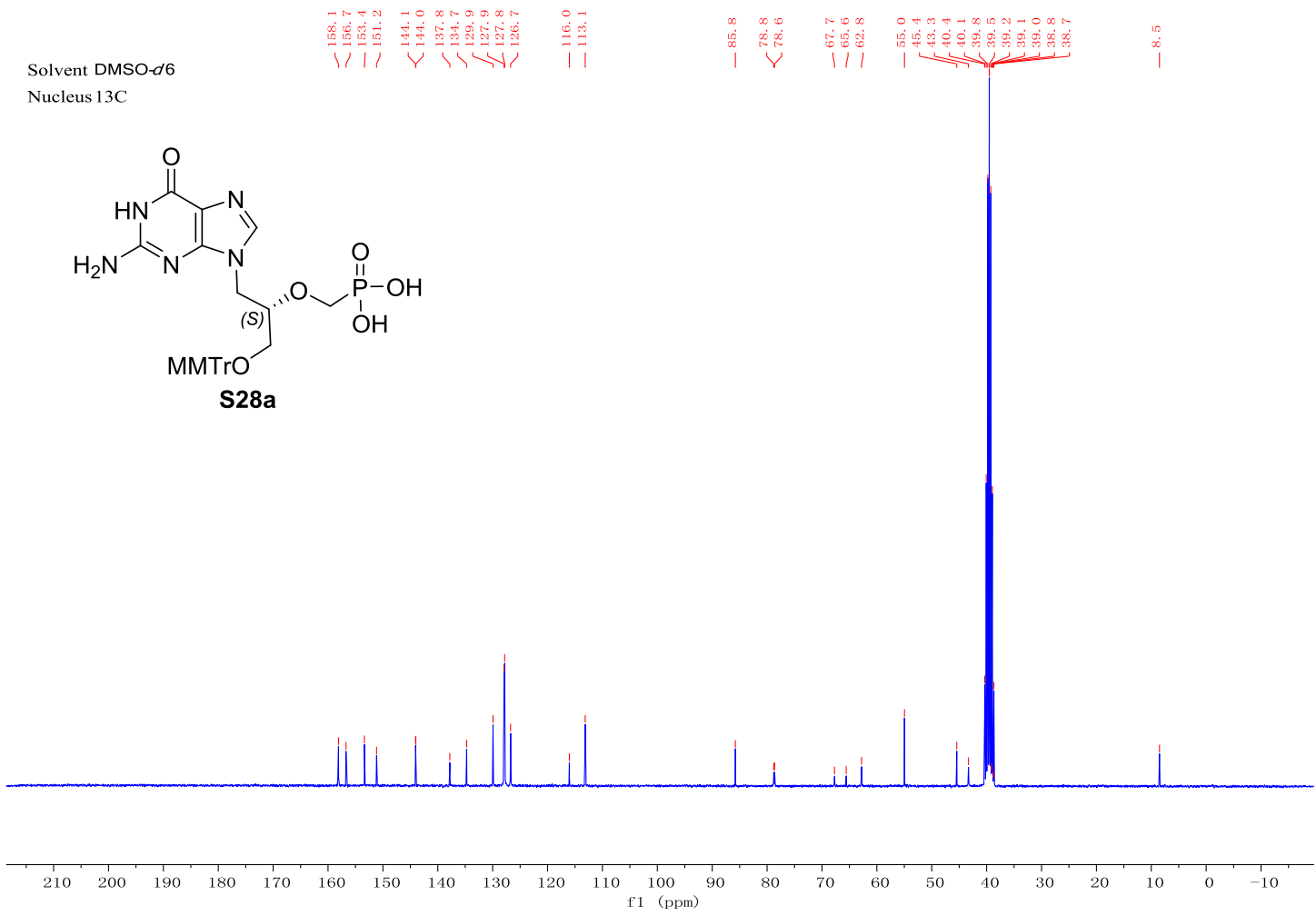

S62 
<smiles>COC[C@H](Cn1cnc2c(=O)[nH]c(N)nc21)OCP(=O)(O)O</smiles>

s28a
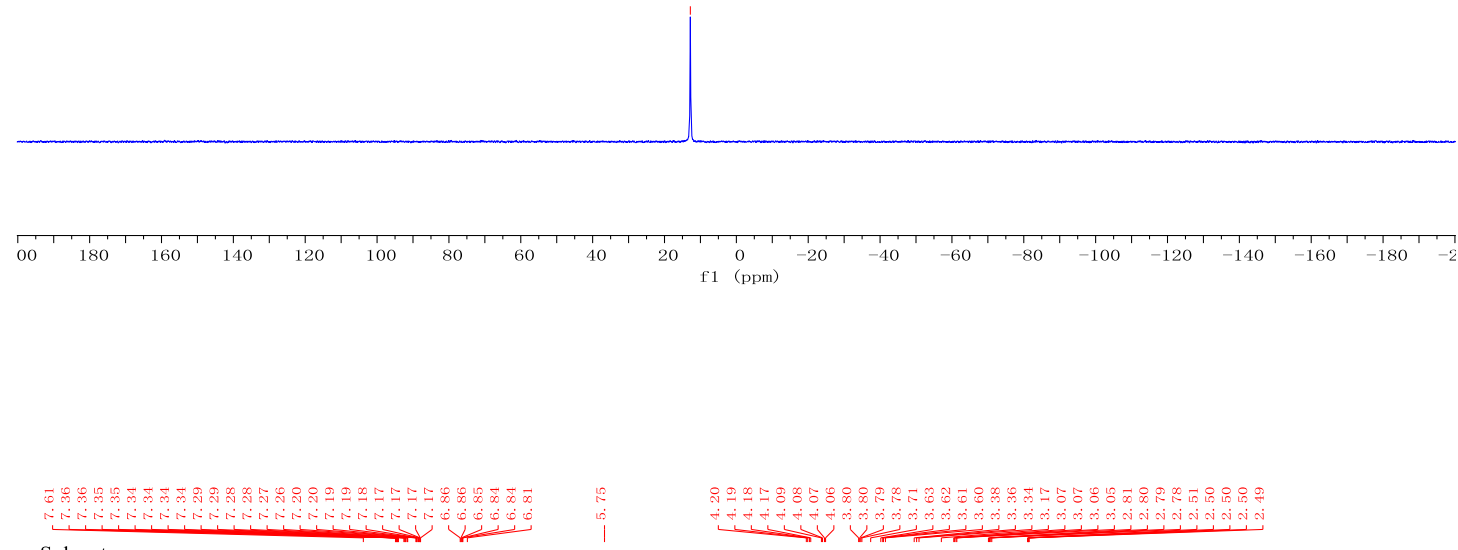
Solvent

Nucleus 1H<smiles>COC[C@H](COP(=O)(O)O)OCn1cnc2c(=O)[nH]c(N)nc21</smiles>

S28b

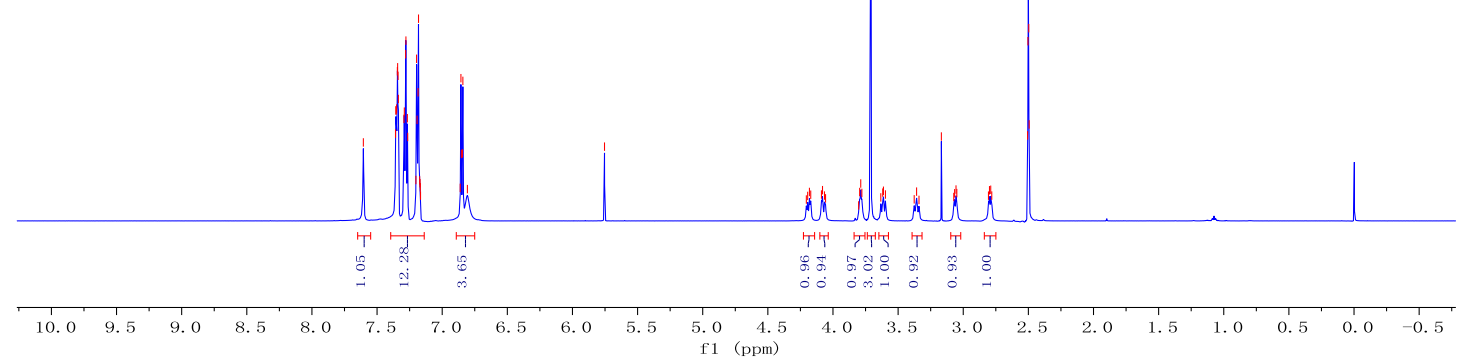


Solvent

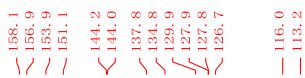

Nucleus $13 \mathrm{C}$<smiles>COC[C@H](COP(=O)(O)O)OCn1cnc2c(=O)[nH]c(N)nc21</smiles>

S28b

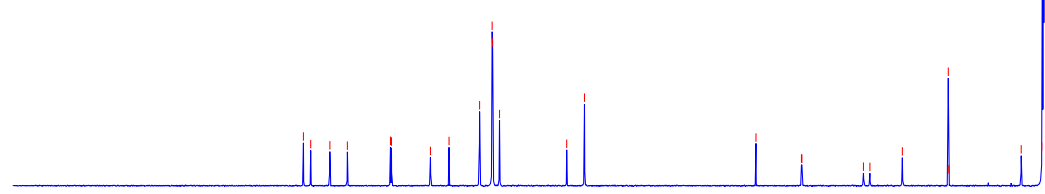

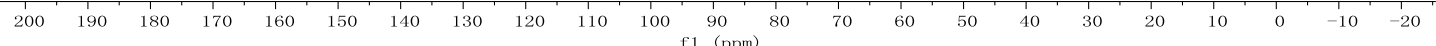

Solvent DMSO-d6

Nucleus $31 \mathrm{P}$

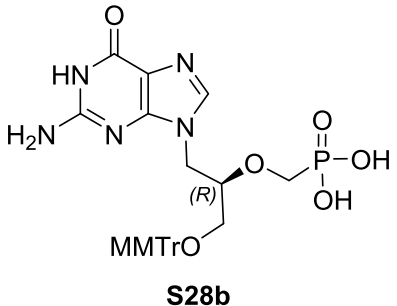

S28b

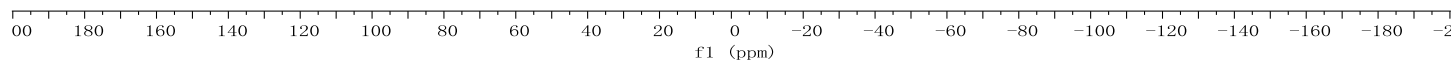




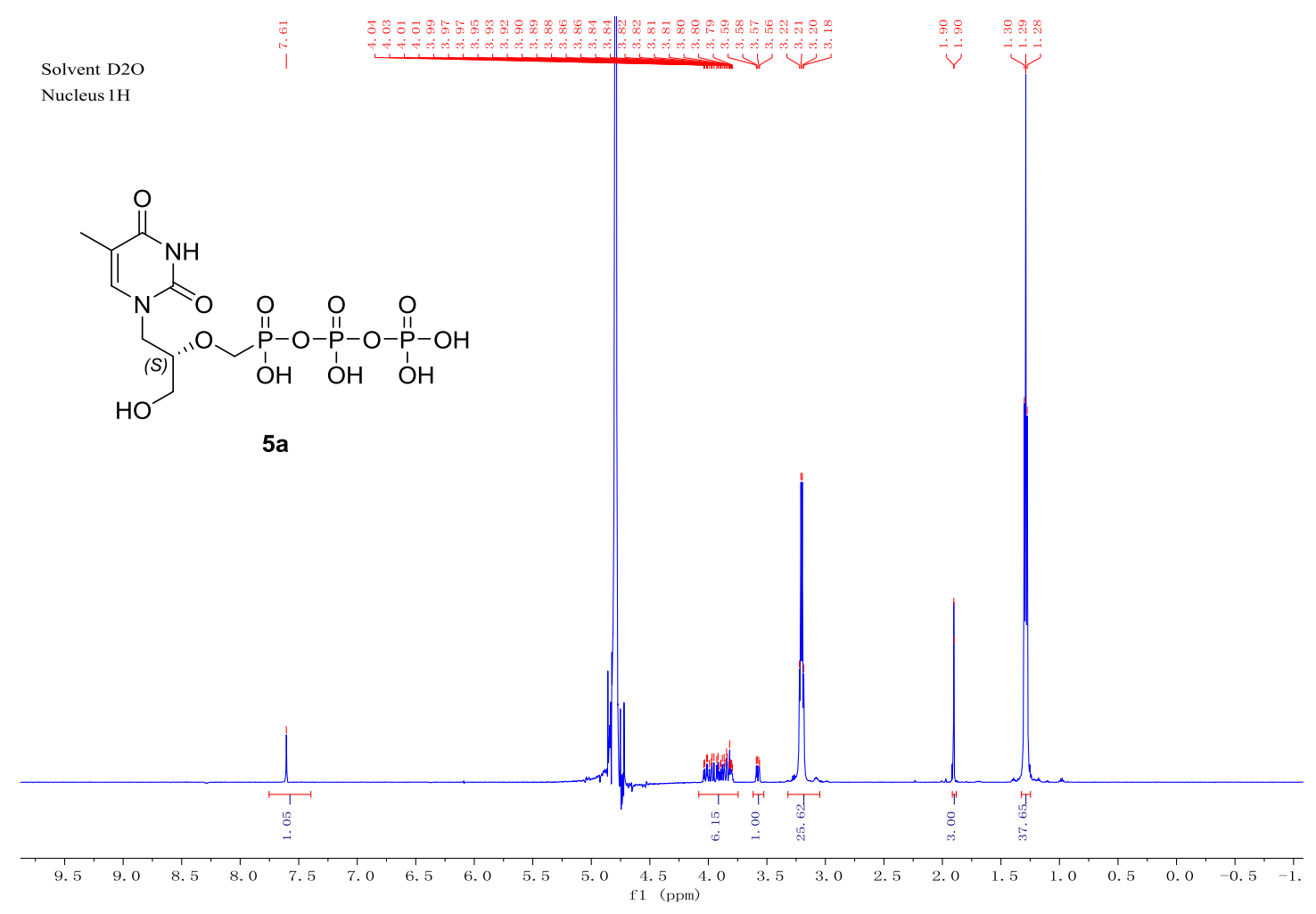

Solvent D2O

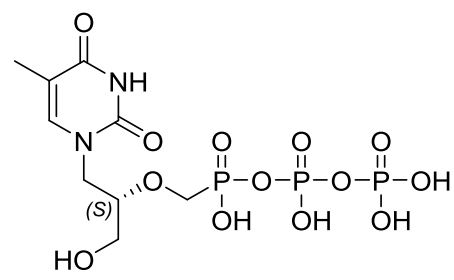

$5 \mathbf{a}$

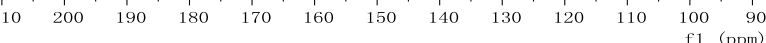


Solvent D2O

Nucleus 31P

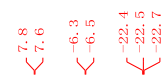<smiles>Cc1cn(C[C@H](CO)OCP(=O)(O)OP(=O)(O)OP(=O)(O)O)c(=O)[nH]c1=O</smiles>

$5 a$

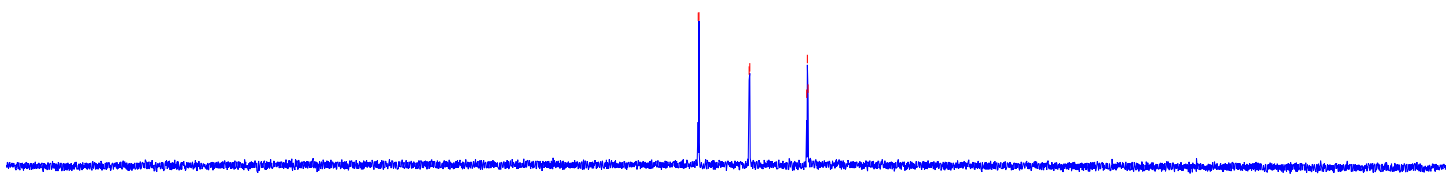

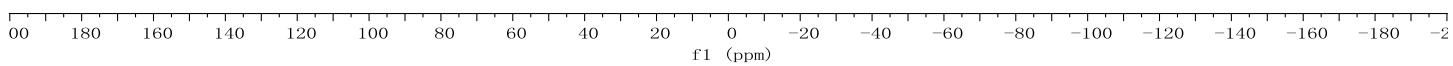

Solvent D2O

Nucleus 1H

$8: 0$
10<smiles>Cc1cn(C[C@@H](CO)OC[P@](=O)(O)OP(=O)(O)OP(=O)(O)O)c(=O)[nH]c1=O</smiles>

$5 \mathbf{b}$

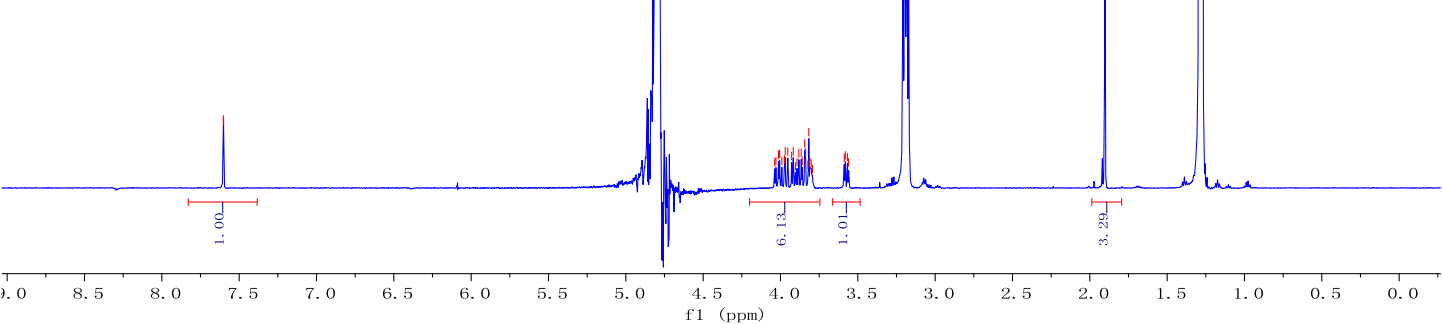

S66 


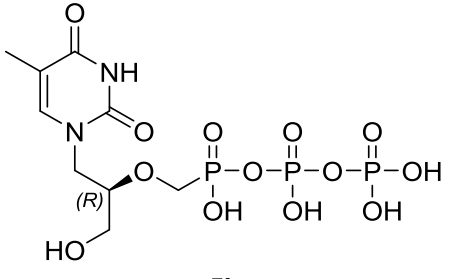

$5 b$

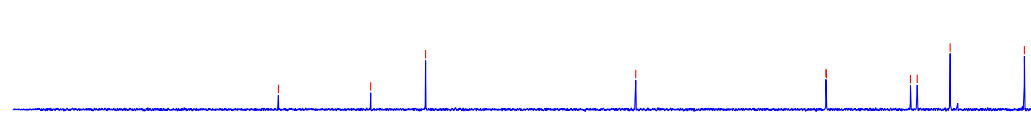

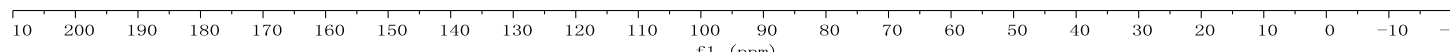

Solvent D2O

Nucleus 31P

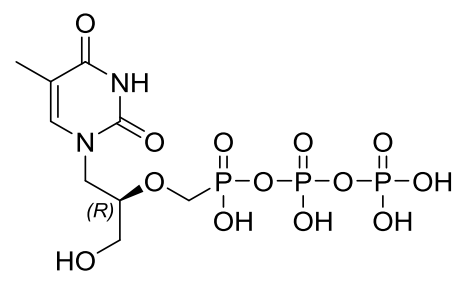

$5 \mathbf{b}$

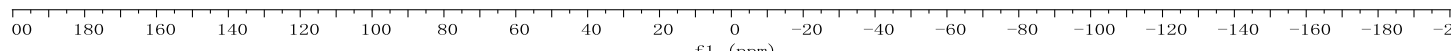



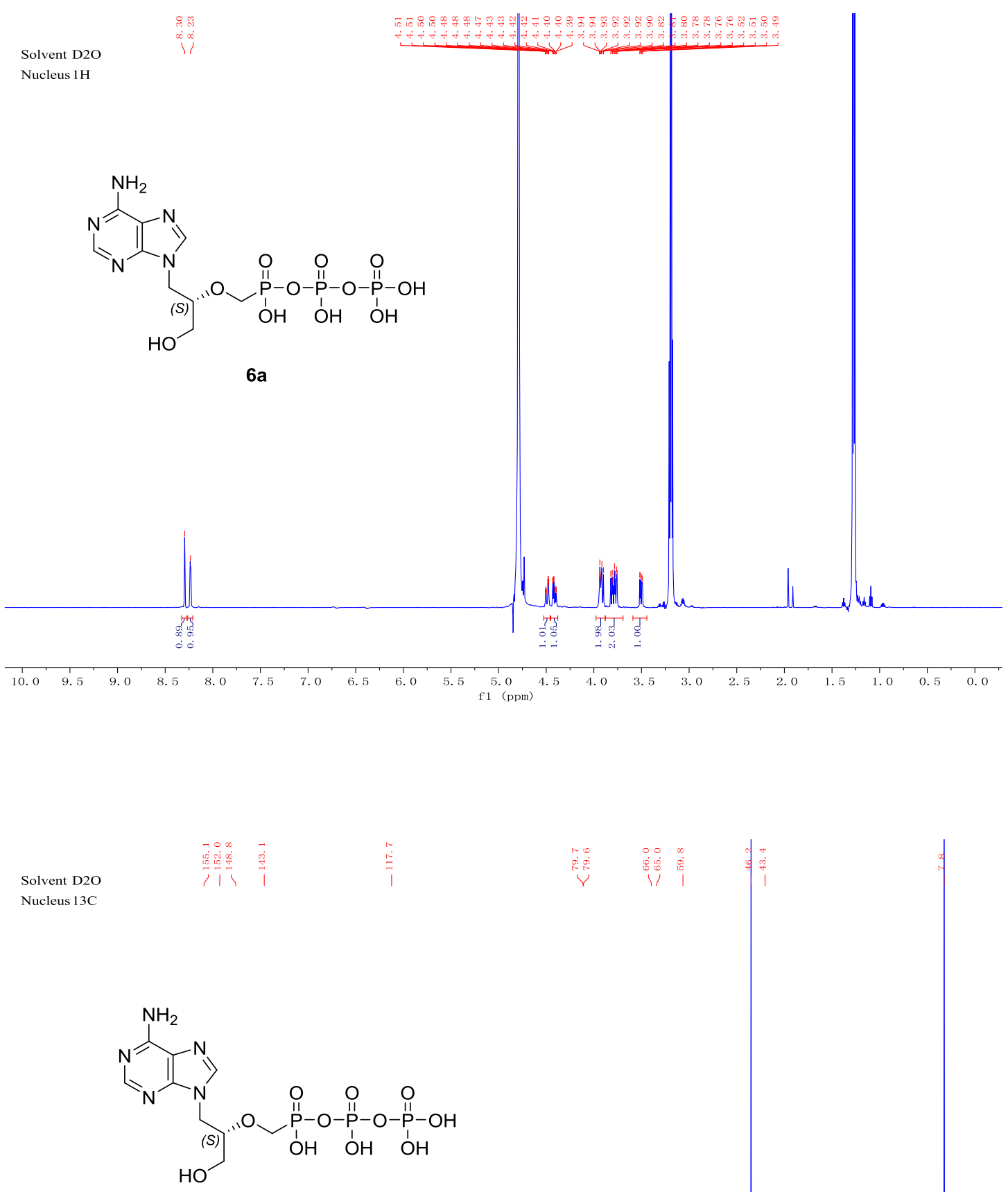

$6 a$ 


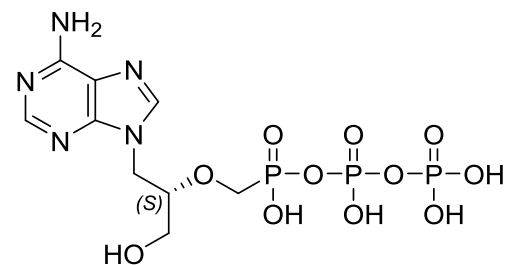

$6 a$

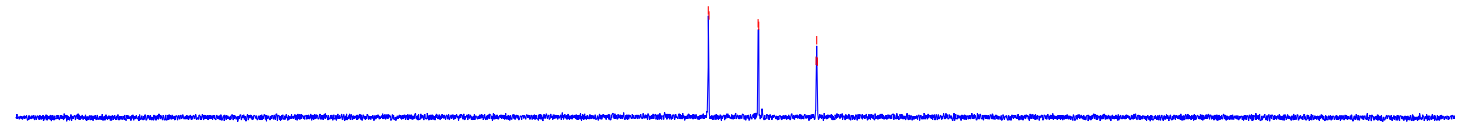

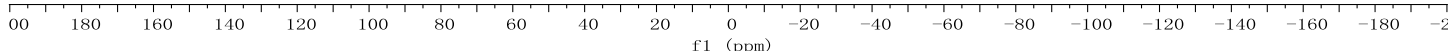

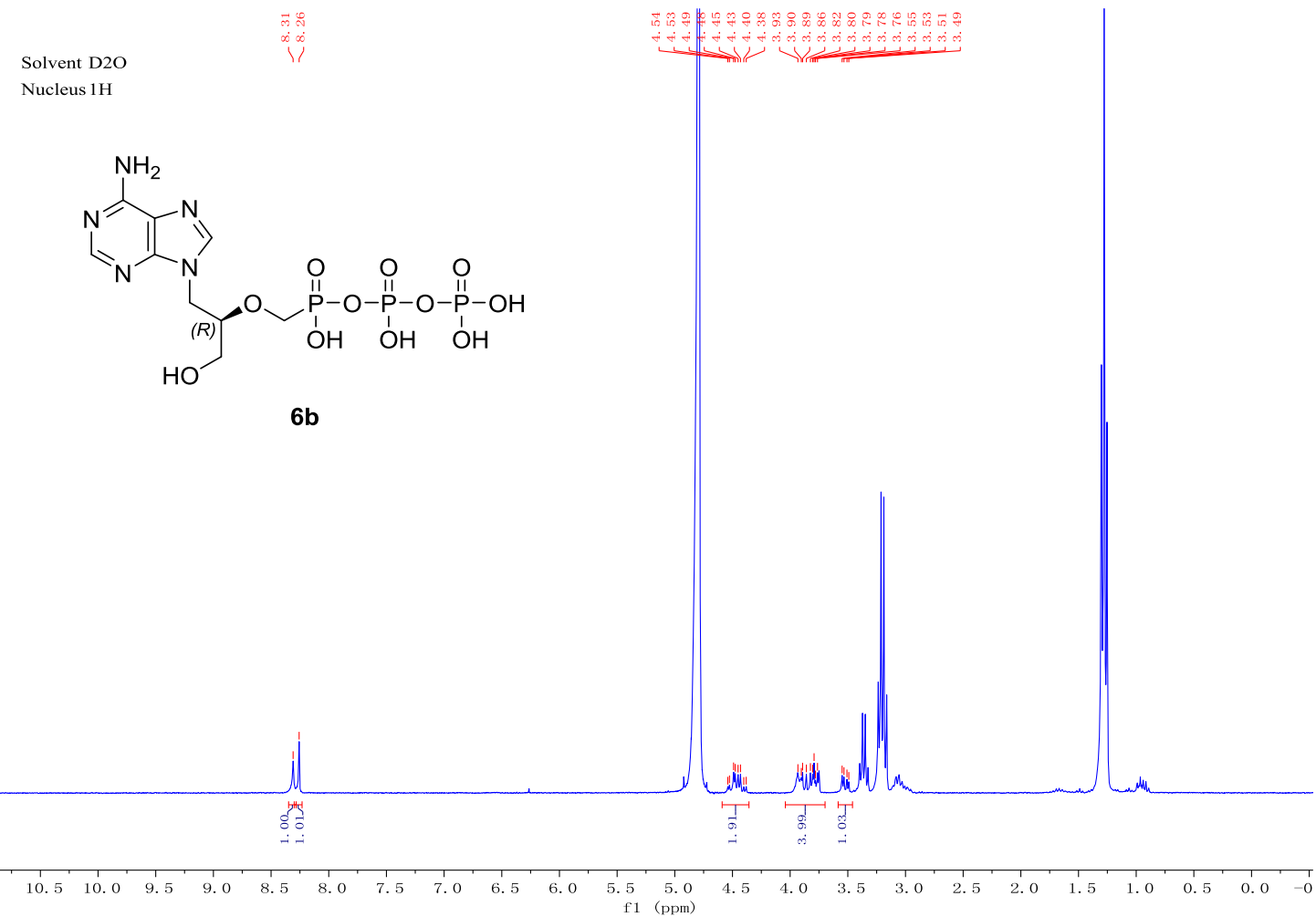




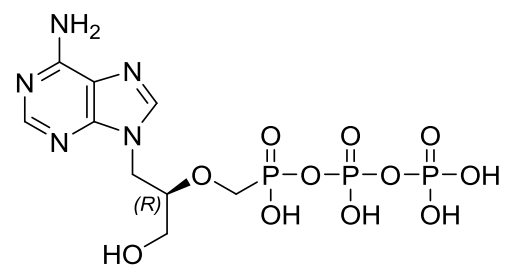

6b

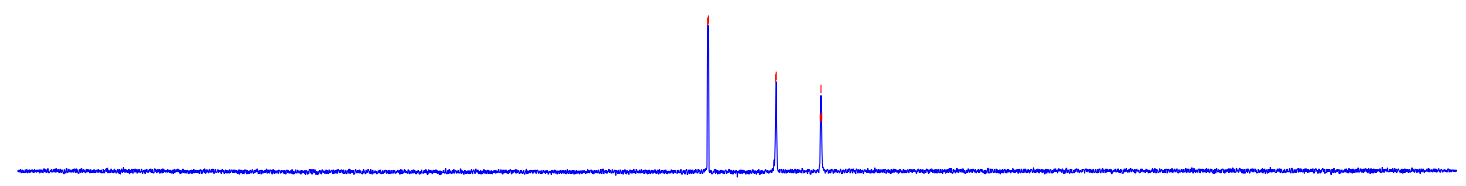

oo 180 

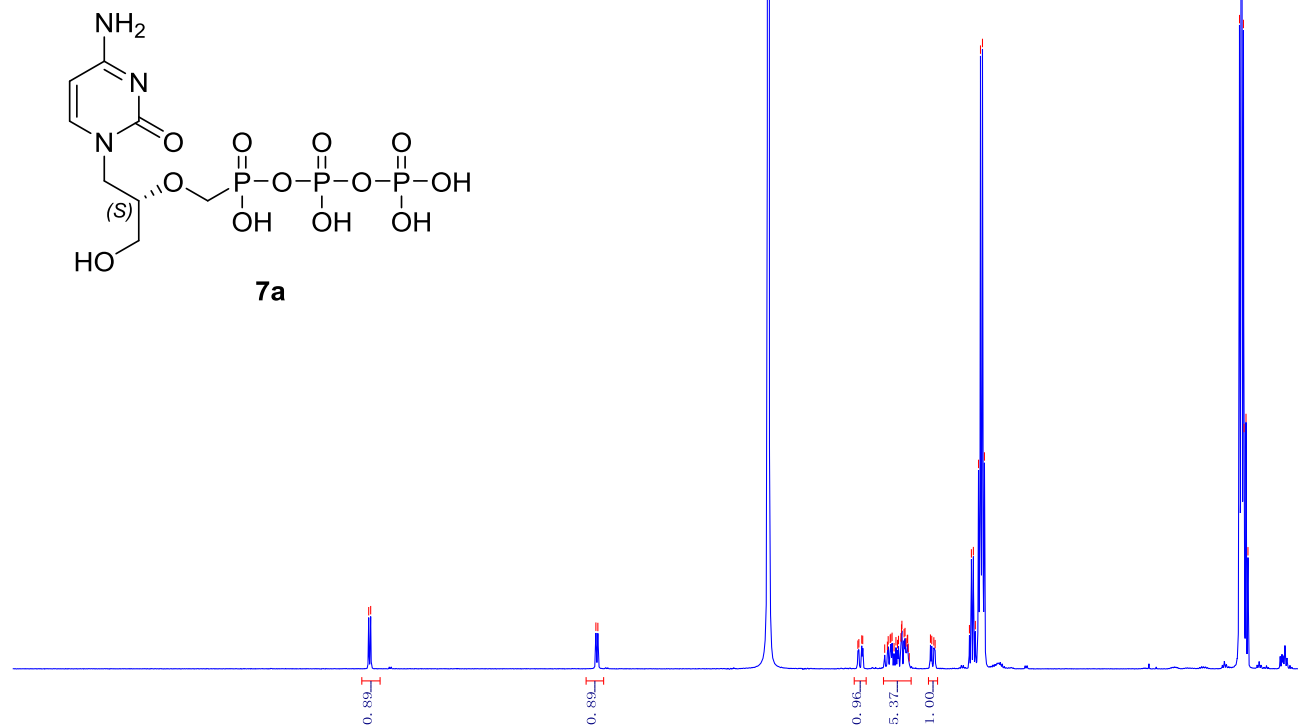

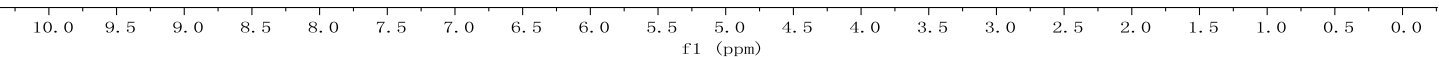

Solvent D2O

Nucleus 13C
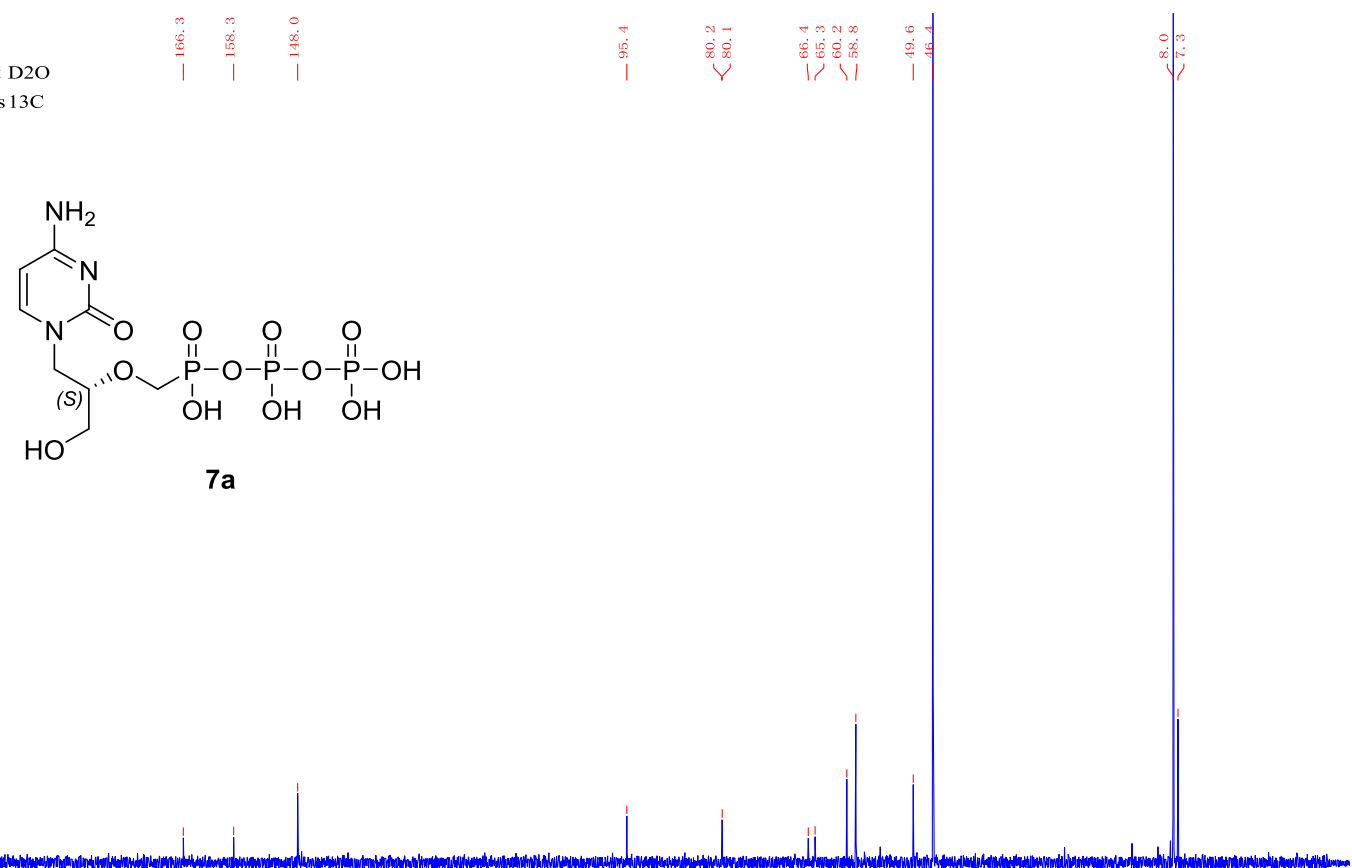

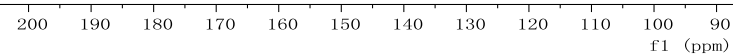



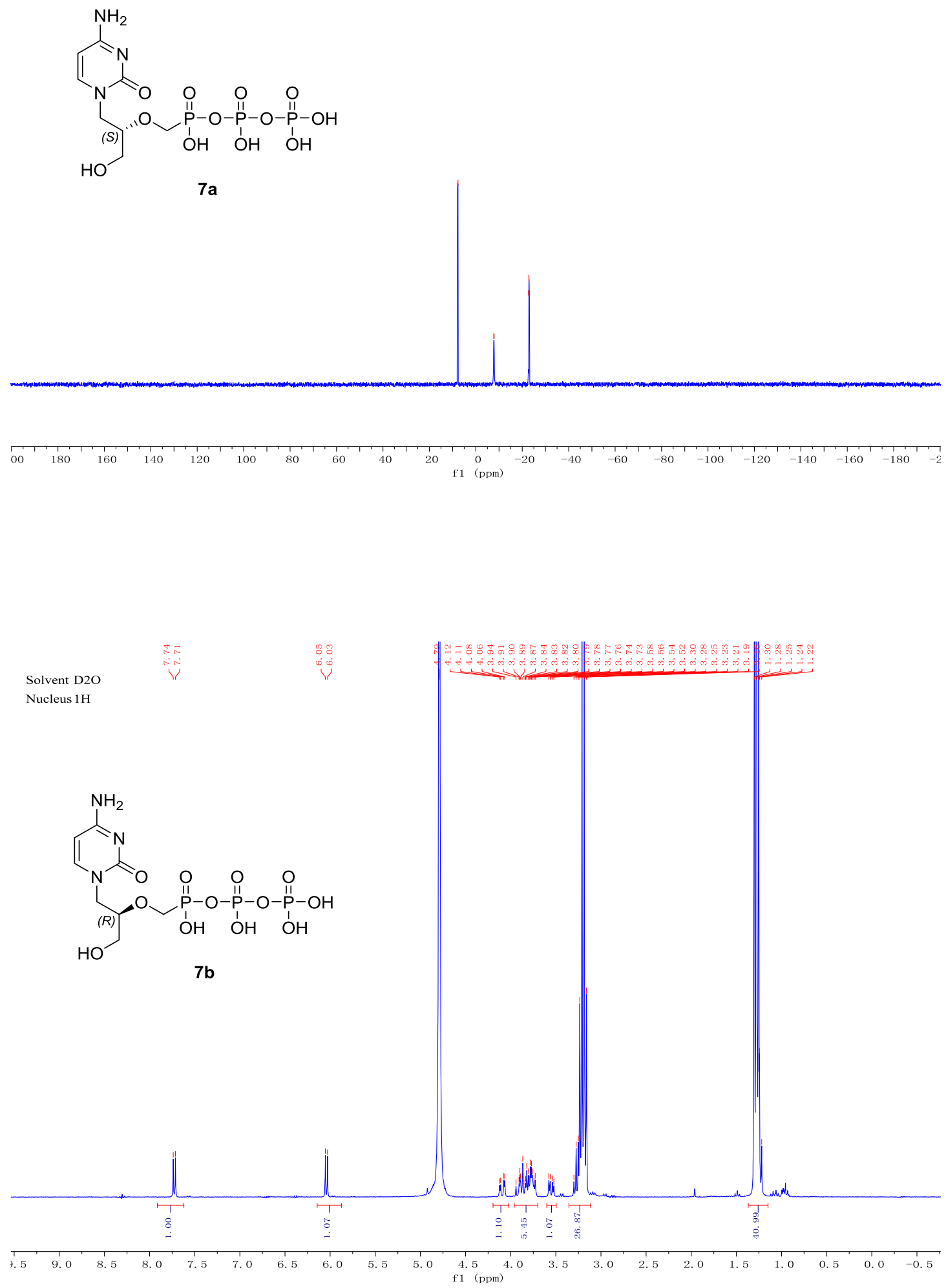


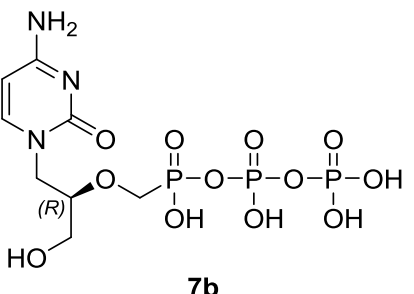

7b

Solvent D2O

Nucleus 31P
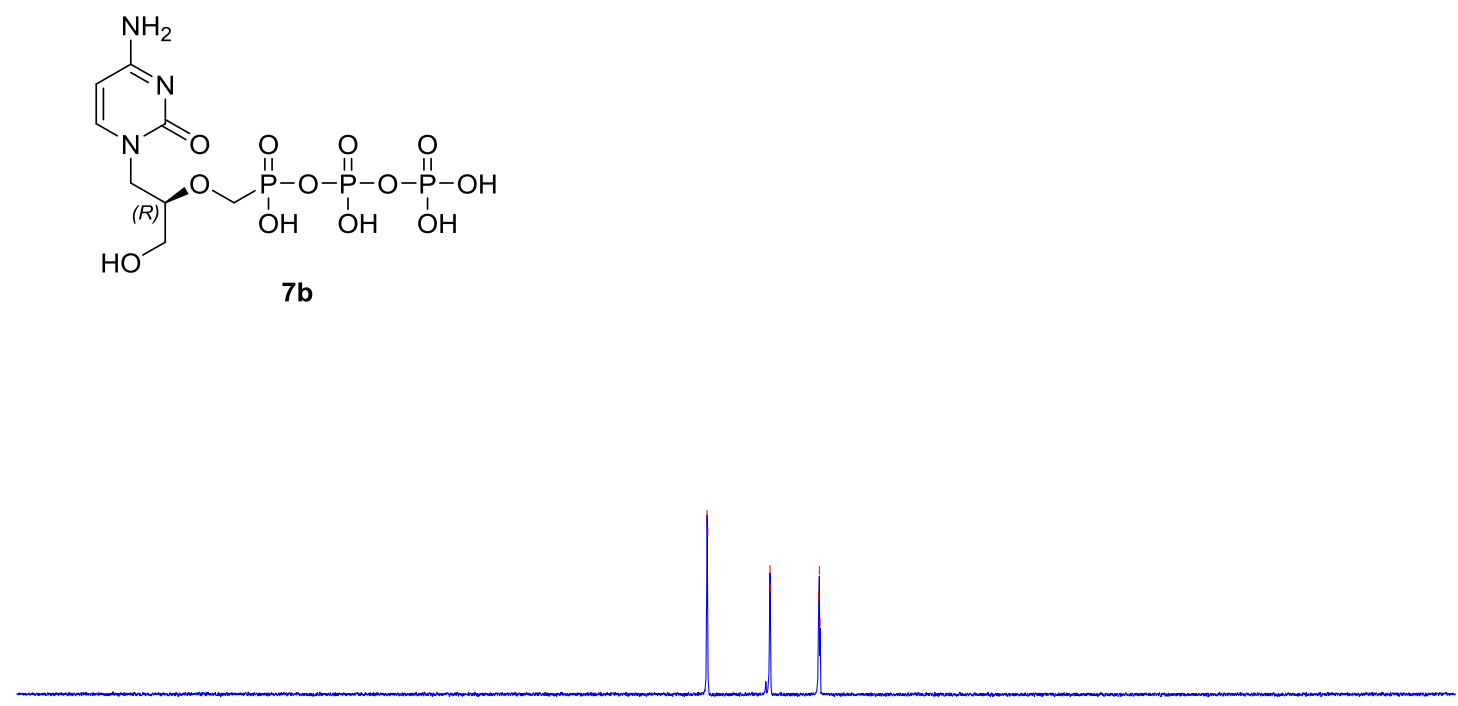

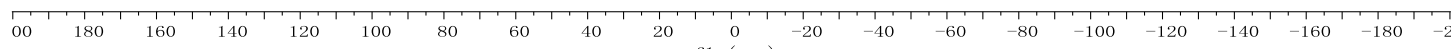



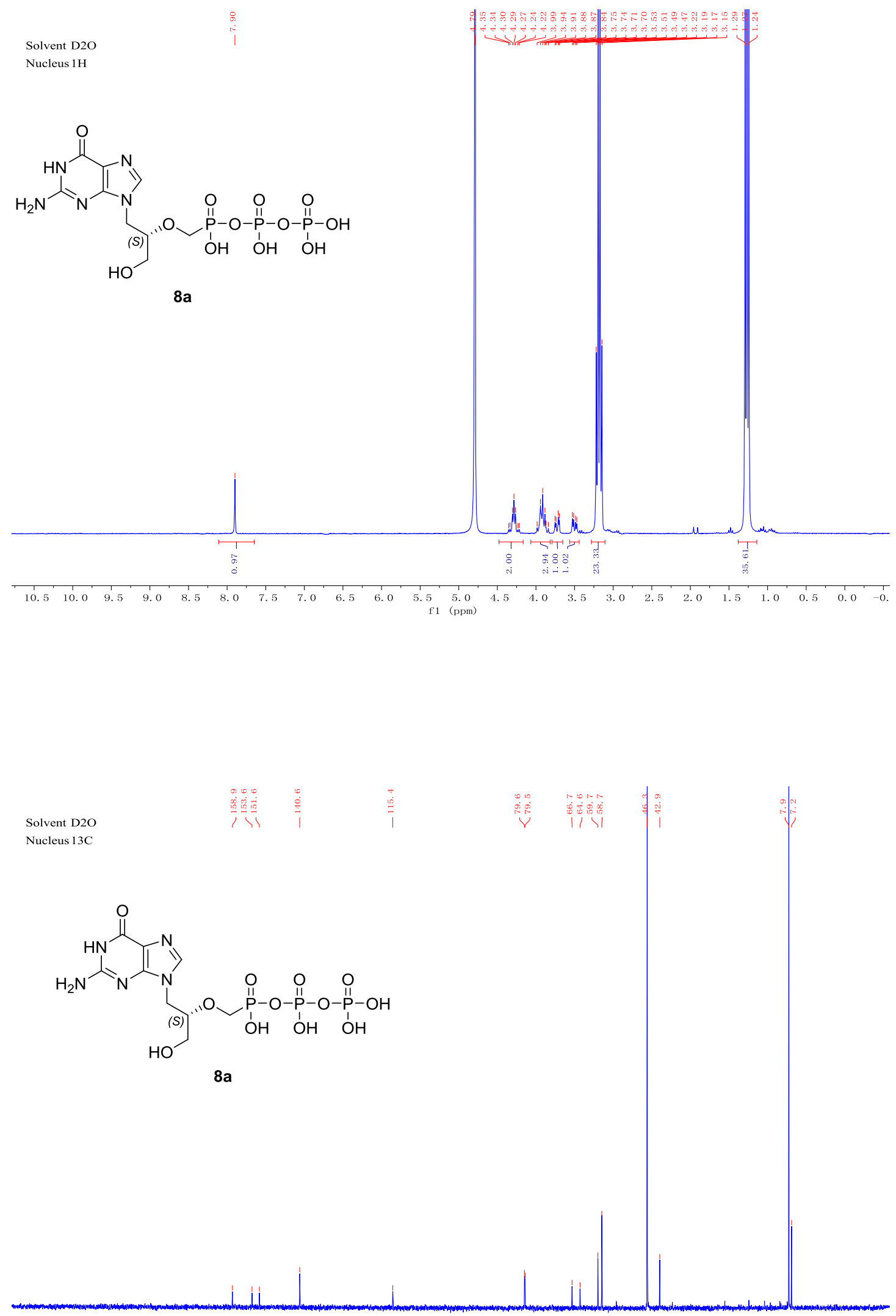

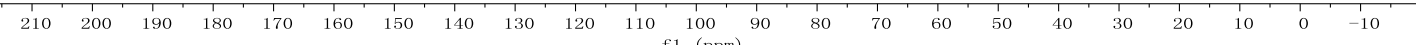




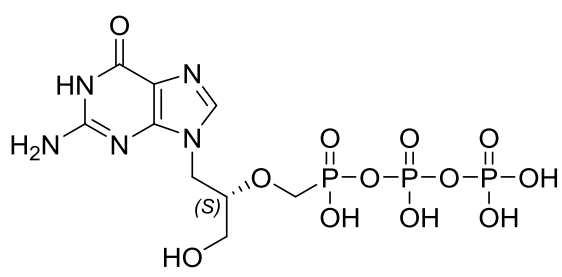

$8 a$

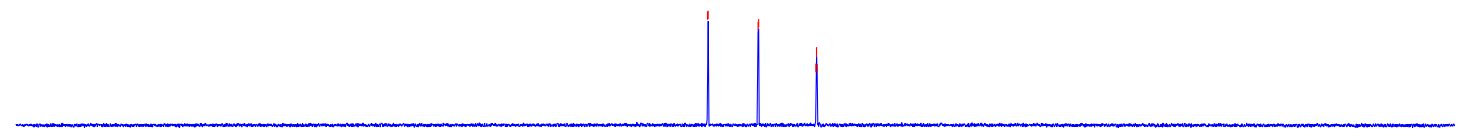

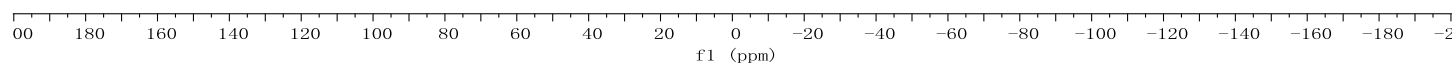

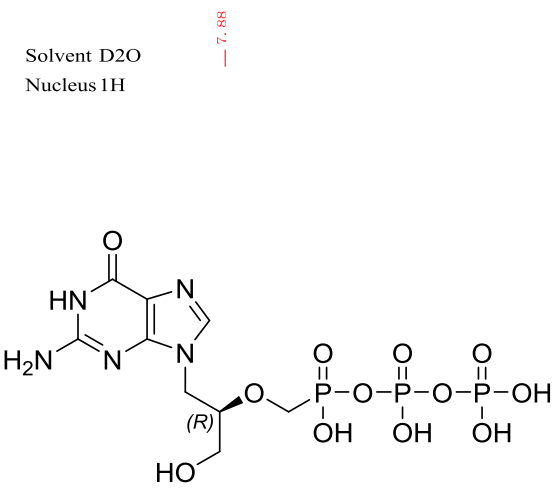

$8 b$

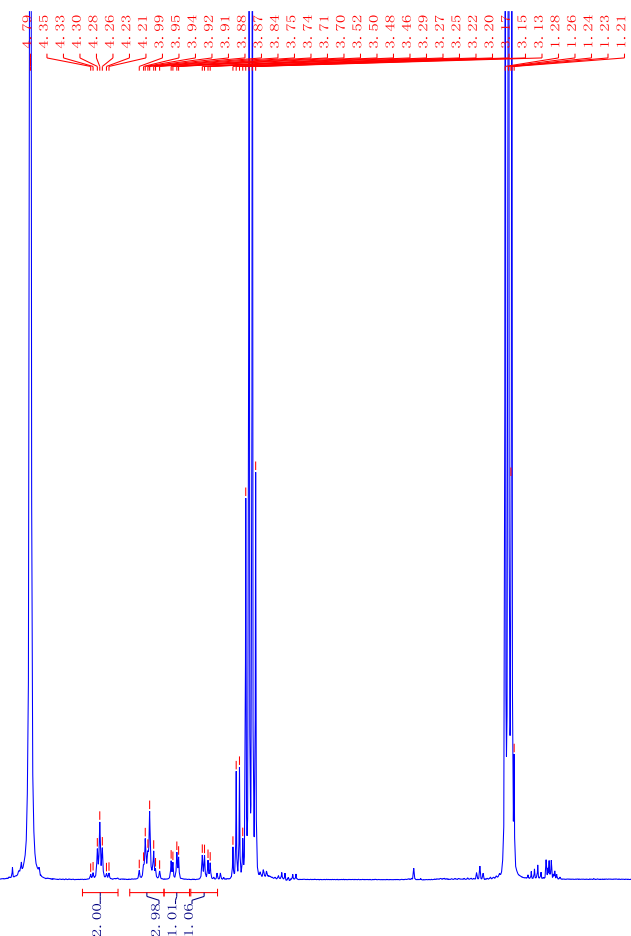

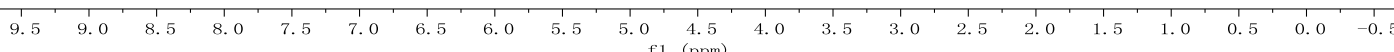




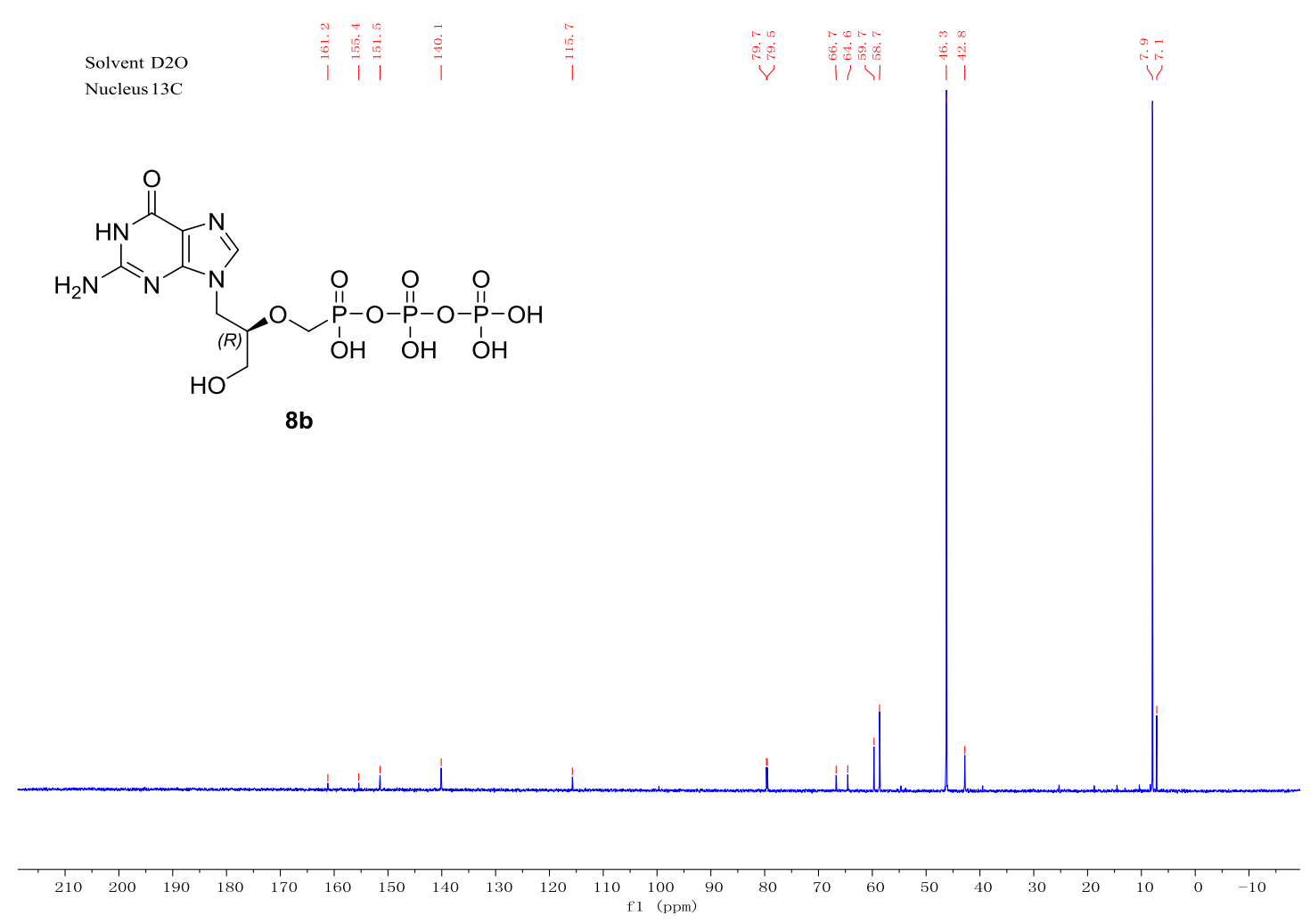

Solvent D2O

Nucleus $31 \mathrm{P}$

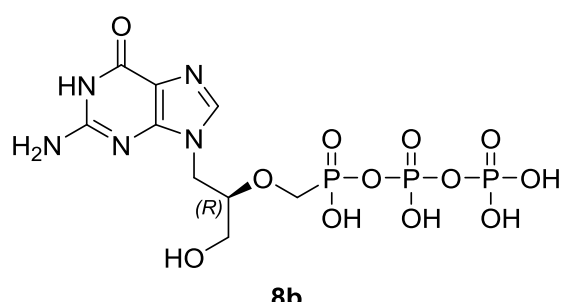

$\mathbf{8 b}$

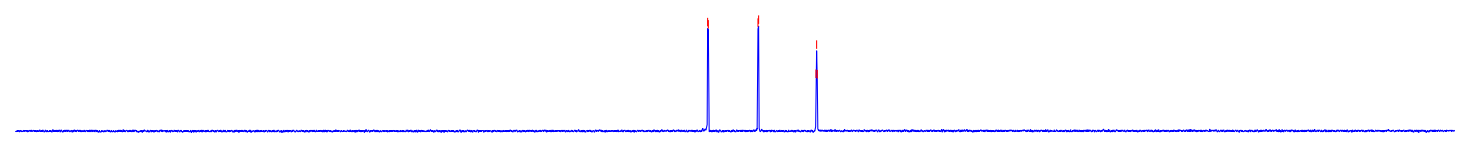

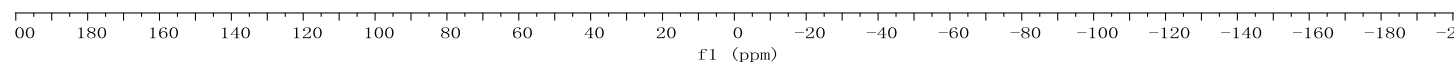




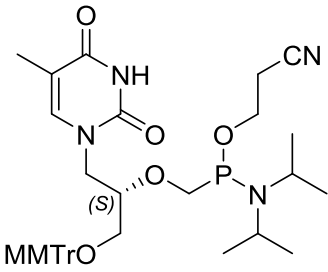

$\mathbf{S 2 9}$

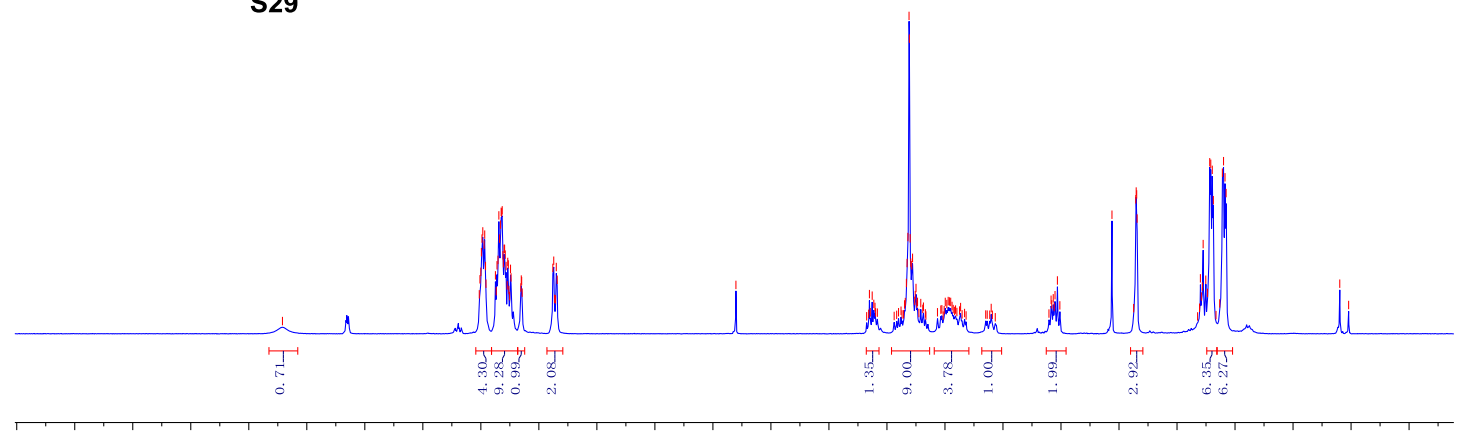

\begin{tabular}{llllllllllllllllllllllllllllllll}
\hline 1.5 & 11.0 & 10.5 & 10.0 & 9.5 & 9.0 & 8.5 & 8.0 & 7.5 & 7.0 & 6.5 & 6.0 & 5.5 & 5.0 & 4.5 & 4.0 & 3.5 & 3.0 & 2.5 & 2.0 & 1.5 & 1.0 & 0.5 & 0.0 & -0.5
\end{tabular}

Solvent CDCl3

Nucleus 31P

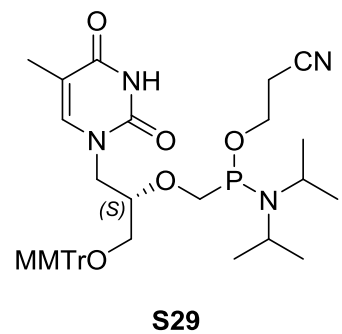

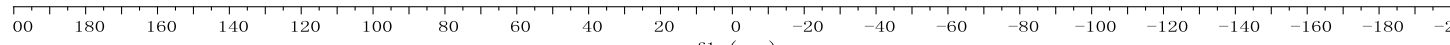




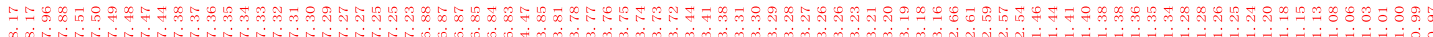
Solvent $\mathrm{CDCl} 3$

Nucleus 1H<smiles>COC[C@H](COP(COCCC#N)N(C(C)C)C(C)C)n1cnc2c(=O)[nH]cnc21</smiles>

$\mathbf{S 3 0}$

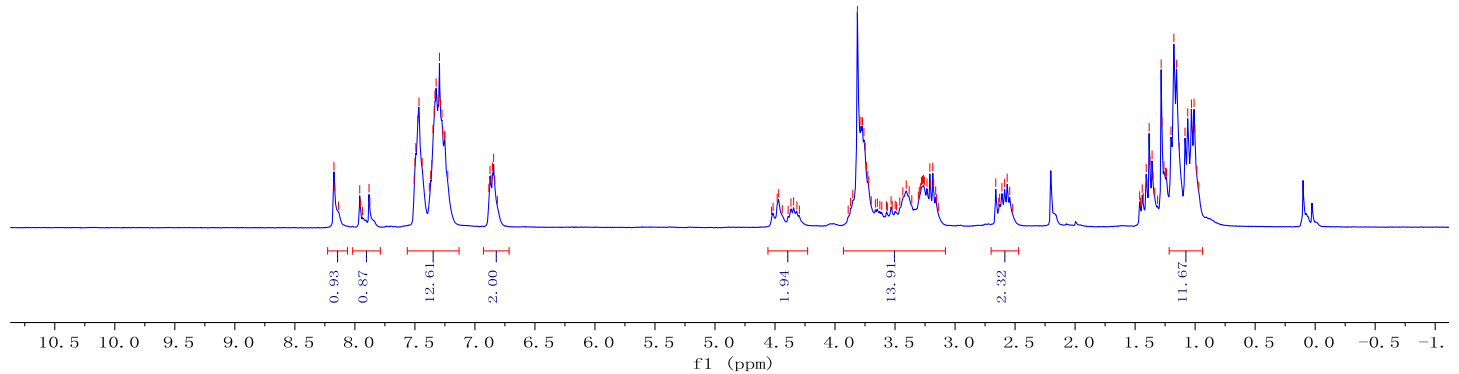

Solvent $\mathrm{CDCl}$

Nucleus 31P<smiles>COC[C@H](COP(CO[C@H](COC)N(C(C)C)C(C)C)Cn1cnc2c(=O)[nH]cnc21)OCCC#N</smiles>

$\mathbf{5 3 0}$

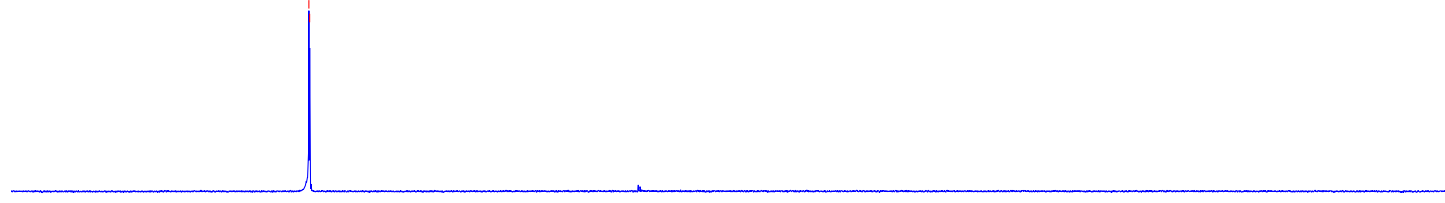

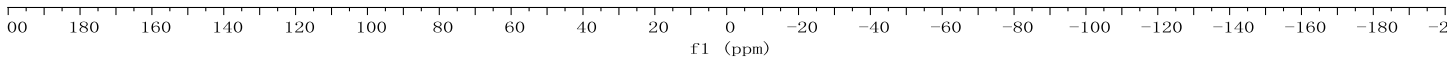

